An Analytical Approach to Efficient Circuit Variability Analysis

in Scaled CMOS Design

by

Samatha Gummalla

A Thesis Presented in Partial Fulfillment of the Requirements for the Degree

Master of Science

Approved May 2011 by the Graduate Supervisory Committee:

Chaitali Chakrabarti, Co-Chair

Yu Cao, Co-Chair

Bertan Bakkaloglu

\title{
ARIZONA STATE UNIVERSITY
}

December 2011 


\begin{abstract}
Process variations have become increasingly important for scaled technologies starting at $45 \mathrm{~nm}$. The increased variations are primarily due to random dopant fluctuations, line-edge roughness and oxide thickness fluctuation. These variations greatly impact all aspects of circuit performance and pose a grand challenge to future robust IC design. To improve robustness, efficient methodology is required that considers effect of variations in the design flow. Analyzing timing variability of complex circuits with HSPICE simulations is very time consuming. This thesis proposes an analytical model to predict variability in CMOS circuits that is quick and accurate.

There are several analytical models to estimate nominal delay performance but very little work has been done to accurately model delay variability. The proposed model is comprehensive and estimates nominal delay and variability as a function of transistor width, load capacitance and transition time. First, models are developed for library gates and the accuracy of the models is verified with HSPICE simulations for $45 \mathrm{~nm}$ and $32 \mathrm{~nm}$ technology nodes. The difference between predicted and simulated $\sigma / \mu$ for the library gates is less than $1 \%$. Next, the accuracy of the model for nominal delay is verified for larger circuits including ISCAS' 85 benchmark circuits. The model predicted results are within 4\% error of HSPICE simulated results and take a small fraction of the time, for $45 \mathrm{~nm}$ technology. Delay variability is analyzed for various paths and it is observed that non-critical paths can become critical because of $V_{t h}$ variation. Variability on shortest paths show that rate of hold violations increase enormously with increasing $V_{t h}$ variation.
\end{abstract}


To my husband Ajith and my friend Gayathri 


\section{ACKNOWLEDGEMENTS}

I would like to express my gratitude and sincere thanks to my advisors Dr. Chaitali Chakrabarti and Dr. Yu Cao for their continuous support and guidance, during the course of the work. I am grateful to Dr. Bertan Bakkaloglu for agreeing to be on my defense committee and for his time and efforts in reviewing my work.

I would like to thank all the members of our lab for their support and encouragement in finishing the thesis. Finally, I take this opportunity to thank my parents, family and friends who have been my pillars of strength through out my career, and who helped me become who I am today.

I gratefully acknowledge the financial support from NSF through CSR0910699. 
LIST OF TABLES $\ldots \ldots \ldots \ldots \ldots \ldots \ldots \ldots \ldots \ldots$ vi

LIST OF FIGURES $\ldots \ldots \ldots \ldots \ldots \ldots \ldots \ldots \ldots$ vii

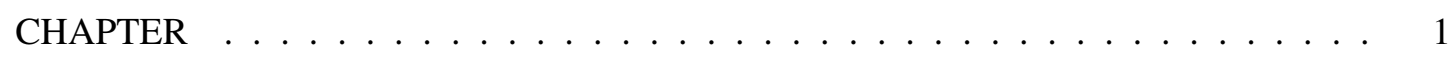

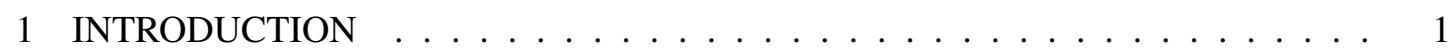

1.1 Motivation ........................ 1

1.2 Existing Work . . . . . . . . . . . . . . . . 1

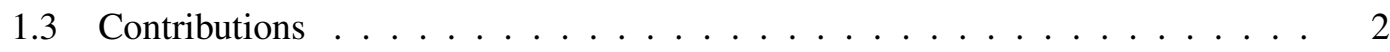

1.4 Thesis Organization . . . . . . . . . . . . . . . . . 3

2 VARIABILITY AND RELIABILITY ANALYSIS . . . . . . . . . . . 4

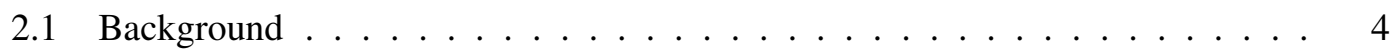

2.2 Variability in Circuit Performance . . . . . . . . . . . . 7

2.2.1 Case Study - Inverter . . . . . . . . . . . . . . . . 7

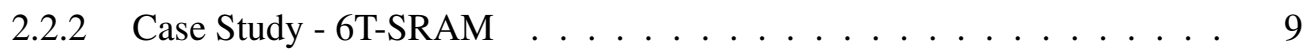

2.3 Effect of Variability on Path length . . . . . . . . . . . . . . . . 13

2.4 Effect of Variation on Logic Style . . . . . . . . . . . . . . . . 16

2.5 Variability and Logical Effort . . . . . . . . . . . . . . . . . . 18

3 ANALYTICAL MODEL FOR NOMINAL DELAY . . . . . . . . . . . . . 20

3.1 Nominal Delay Model for Inverter . . . . . . . . . . . . . . . . . . 20

3.1 .1 Model derivation . . . . . . . . . . . . . . . . . 21

3.1 .2 Model Validation . . . . . . . . . . . . . . . . . 24

3.2 Nominal Delay Model for NAND and NOR gates . . . . . . . . . . . 26

3.2.1 NAND2 Delay Model . . . . . . . . . . . . . . 26

3.2 .2 Model Validation . . . . . . . . . . . . . . . 30

3.2.3 NAND3 Delay Model . . . . . . . . . . . . . . . . 33

3.2 .4 NAND3 Validation . . . . . . . . . . . . . . . . . 34

3.2 .5 Summary: . . . . . . . . . . . . . . 35

4 ANALYTICAL MODEL FOR DELAY VARIABILITY . . . . . . . . . . . 38

4.1 Delay Variability in Inverter f . . . . . . . . . . . . . 38

4.2 Delay Variability in NAND2 and NOR2 gates . . . . . . . . . . . . . . . 42 
4.3 Delay Variability in NAND3 . . . . . . . . . . . . . . . . . . . . 44

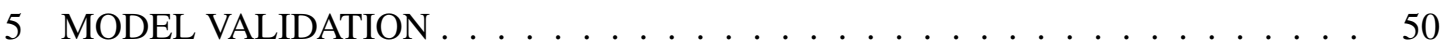

5.1 Small Circuits . . . . . . . . . . . . . . . . . . 50

5.2 Application to ISCAS Benchmark Circuits . . . . . . . . . . . . . 52

5.2 .1 Effects of Variability . . . . . . . . . . . . . 54

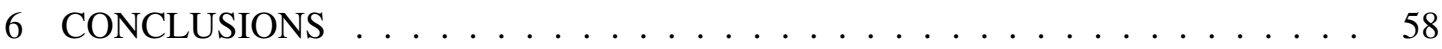

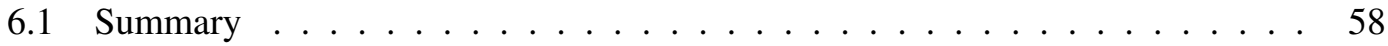

6.2 Future Work . . . . . . . . . . . . . . . . . . . . . . 59

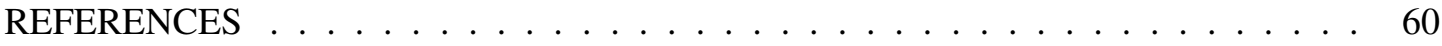




\section{LIST OF TABLES}

Table

Page

2.1 Minimum length, $V_{D D}$, and p-n ratios of inverter for different technologies. . . . . 8

2.2 SRAM transistor widths when length is taken to be minimum. . . . . . . . . . 10

2.3 Nominal delay and delay variation when AND6 is implemented in different styles at $45 \mathrm{~nm}$ technology node. . . . . . . . . . . . . . . . . . . . . 17

2.4 Nominal delay and delay variation when AND6 is implemented in different styles at $12 \mathrm{~nm}$ technology node. . . . . . . . . . . . . . . . . . . 18

2.5 Nominal delay and delay variation of buffer stage driving $1 \mathrm{pf}$ load with different number of stages at $12 \mathrm{~nm}$ technology node. . . . . . . . . . . . . . . . . . . 19

3.1 Parameters used in the model and their extraction information. . . . . . . . . . . 37

4.1 Variation numbers when input is given to top(M1) and bottom(M2) transistors of NAND2 gate, with $V_{t h}$ of one of them varying. . . . . . . . . . . . . . . . . 44

4.2 Variation numbers when input is given to top(M1), middle(M2) and bottom(M3) transistors of NAND3 gate, with $V_{t h}$ of one of them varying. . . . . . . . . . . 47

5.1 XOR2 gate nominal delay and delay variation values from HSPICE simulations and model estimates. . . . . . . . . . . . . . . . . . 50

5.2 Full Adder nominal delay and delay variation values from simulated results and model estimated results. . . . . . . . . . . . . . . . . . . . . . 52

5.3 Comparison of nominal delay estimation for all the ISCAS' 85 benchmark circuits. 53

5.4 Variation prediction for critical paths in ISCAS' 85 benchmark circuits . . . . . . 54 


\section{LIST OF FIGURES}

Figure

Page

2.1 Effect of inter-die and intra-die variations in NAND2-RO delay, at different technologies. ....................... 5

2.2 Effect of inter-die and intra-die variations in 6T-SRAM DRV, at different technologies. 5

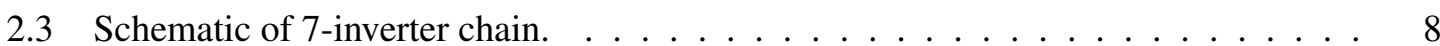

2.4 Inverter: Mean delay and sigma as percentage of mean delay. . . . . . . . . . . . 8

2.5 Inverter delay variation due to each intrinsic factor. . . . . . . . . . . . . . . 9

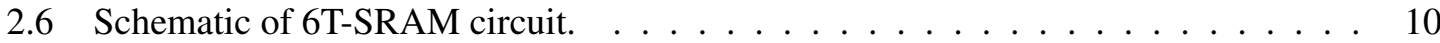

2.7 SRAM: Comparison of access time PDF's in 45nm, 22nm, and 12nm technologies. 11

2.8 SRAM. Mean and $3 \sigma$ point for all technologies $\ldots \ldots \ldots \ldots$. . . . . . . . 11

2.9 SRAM RNM variability due to each intrinsic factor variation. . . . . . . . . . . . . 13

2.10 Nominal Delay and Delay variation with different path lengths at $45 \mathrm{~nm}$ technology node at (a) nominal voltage of $V_{D D}=1.0 \mathrm{~V}$, (b) $V_{D D}=0.5 \mathrm{~V} \ldots \ldots \ldots \ldots$

2.11 Nominal Delay and Delay variation with different path lengths at 22nm technology at (a) nominal voltage of $V_{D D}=0.8 \mathrm{~V}$, (b) $V_{D D}=0.5 \mathrm{~V} \ldots \ldots \ldots \ldots \ldots$

2.12 Nominal Delay and Delay variation with different path lengths at $12 \mathrm{~nm}$ technology at (a) nominal voltage of $V_{D D}=0.65 \mathrm{~V}$, (b) $V_{D D}=0.5 \mathrm{~V} \ldots \ldots \ldots \ldots$

2.13 Different implementations of AND6 function. . . . . . . . . . . . . . . . 17

2.14 Buffer loaded with $1 \mathrm{pF}$ capacitance. . . . . . . . . . . . . . . . . 18

3.1 NMOS characteristics - Simulated and Analytical . . . . . . . . . . . . . . . . 21

3.2 Schematic of CMOS Inverter circuit. . . . . . . . . . . . . . . . . . . 21

3.3 Regions of operation of NMOS transistor as input rises. . . . . . . . . . . . . . 22

3.4 Inverter HL delay with varying width, capacitance, transition time at $45 \mathrm{~nm}$ technology node. . . . . . . . . . . . . . . . . . . 25

3.5 Inverter HL delay with varying width, capacitance, transition time at $32 \mathrm{~nm}$ technology node. . . . . . . . . . . . . . . . . . . . 25

3.6 Inverter LH delay with varying width, capacitance, transition time at $45 \mathrm{~nm}$ technology node. . . . . . . . . . . . . . . . . . . . 26

3.7 Inverter LH delay with varying width, capacitance, transition time at $32 \mathrm{~nm}$ technology node. . . . . . . . . . . . . . . . . . . . . 27 
Figure Page

3.8 NAND2 gate schematics. . . . . . . . . . . . . . . . . . . . . 27

3.9 NAND2 gate discharge behavior when input is given to bottom transistor. . . . . . 28

3.10 NAND2 gate HL delay with varying width, capacitance, transition time when input is given to $\mathrm{M} 2$ at $45 \mathrm{~nm}$ technology node. . . . . . . . . . . . . . . 30

3.11 NAND2 gate HL delay with varying width, capacitance, transition time when input is given to $\mathrm{M} 1$ at $45 \mathrm{~nm}$ technology node. . . . . . . . . . . . . . . 31

3.12 NOR2 gate LH delay with varying width, capacitance, transition time when input is given to bottom PMOS at $45 \mathrm{~nm}$ technology node. . . . . . . . . . . . . 32

3.13 NOR2 gate LH delay with varying width, capacitance, transition time when input is given to top PMOS at $45 \mathrm{~nm}$ technology node. . . . . . . . . . . . . 32

3.14 NAND3 gate schematics. . . . . . . . . . . . . . . . . . . 33

3.15 NAND3 gate HL delay with varying width, capacitance, transition time when input is given to M1 at $45 \mathrm{~nm}$ technology node. . . . . . . . . . . . . . 35

3.16 NAND3 gate HL delay with varying width, capacitance, transition time when input is given to $\mathrm{M} 2$ at $45 \mathrm{~nm}$ technology node. . . . . . . . . . . . . . 36

3.17 NAND3 gate HL delay with varying width, capacitance, transition time when input is given to M3 at $45 \mathrm{~nm}$ technology node. . . . . . . . . . . . . . . . 36

4.1 Inverter HL delay variation with varying (a) width, (b) capacitance, (c) transition time at $45 \mathrm{~nm}$ technology node. . . . . . . . . . . . . . . . . . . 39

4.2 Inverter HL delay variation with varying (a)width, (b)capacitance, (c)transition time at $32 \mathrm{~nm}$ technology node. . . . . . . . . . . . . . . . . . . . 40

4.3 Inverter LH delay variation with varying width, capacitance, transition time at $45 \mathrm{~nm}$ technology node. . . . . . . . . . . . . . . . . .

4.4 Inverter LH delay variation with varying width, capacitance, transition time at $32 n m$ technology node. . . . . . . . . . . . . . . . .

4.5 NAND2 gate HL delay variation with varying width, capacitance, transition time when input is given to $\mathrm{M} 2$ at $45 \mathrm{~nm}$ technology node. . . . . . . . . . . . . .

4.6 NAND2 gate HL delay variation with varying width, capacitance, transition time when input is given to M1 at $45 \mathrm{~nm}$ technology node. 
4.7 NAND3 gate HL delay variation with varying width, capacitance, transition time when input is given to $\mathrm{M} 1$ at $45 \mathrm{~nm}$ technology node. . . . . . . . . . . . . . 48

4.8 NAND3 gate HL delay variation with varying width, capacitance, transition time when input is given to $\mathrm{M} 2$ at $45 \mathrm{~nm}$ technology node. . . . . . . . . . . . . . 48

4.9 NAND3 gate HL delay variation with varying width, capacitance, transition time when input is given to $\mathrm{M} 3$ at $45 \mathrm{~nm}$ technology node. . . . . . . . . . . . . . 49

5.1 Schematic of XOR2 circuit $\ldots \ldots \ldots \ldots \ldots \ldots$

5.2 XOR2 gate with input and output loading with FO4 . . . . . . . . . . . . 51

5.3 Mirror Adder structure of Full Adder . . . . . . . . . . . . . . . . . . . . 52

5.4 Full Adder with input and output loading with FO4 . . . . . . . . . . . . . 52

5.5 Delay distribution curve for $\mathrm{C} 880$ benchmark circuit at nominal and with variations. 55

5.6 Non-critical path becoming critical in light of $V_{t h}$ variation. . . . . . . . . 56

5.7 Number of paths that can cause hold time violations because of $V_{t h}$ variations in (a) C5315, (b) C2670, ISCAS benchmark circuit at 45nm technology node. . . . . . . 57 


\section{Chapter 1}

\section{INTRODUCTION}

\subsection{Motivation}

As CMOS technology nodes move to $45 \mathrm{~nm}$ and below, process variations increase significantly. This causes high variability in circuit performance and also reduces manufacturing yield. Various techniques like global back gate biasing and adaptive $V_{D D}$ have been proposed to reduce variation $[10,5]$. But these techniques can correct only small amounts of variation. To improve manufacturing yield of technologies $45 \mathrm{~nm}$ and below, performance variability should be considered during the design phase. In the conventional design approach, high variability leads to over designing, thereby increasing area and power consumption. To avoid over designing, accurate estimation of variability is required. Estimating variability in complex circuits using HSPICE is impractical because of large simulation time for even moderate sized circuits. Also number of paths to be analyzed increase with complexity of the circuit and it becomes practically impossible to analyze all of them with HSPICE simulations.

In this thesis, an analytical model has been proposed to accurately predict variability for any number of paths. While there are many analytical models $[25,26,31]$ to predict nominal delays, these models do not analyze effect of process variations, which is critical for future technology nodes. The existing work on variability analysis are either not accurate [6] or do not provide analytical models for fast estimation [20]. In contrast, this thesis proposes a model that is very accurate and provides a fast way to analyze variability in complex CMOS circuits.

\subsection{Existing Work}

Estimating delay analytically is important in circuit design because it gives insights into the factors affecting delay and gives the designer better control over the design. Of all the models for delay estimation, Shockley's model [25] is the most widely used one. But for submicron technologies, Shockley's delay model is not accurate because it does not consider the effect of velocity saturation. Sakurai and Newton's [26] $\alpha$-power model, on the other hand, considers velocity saturation and is simple and accurate. But the $\alpha$-power law model does not consider channel length modulation. Current equations considering channel length modulation have been developed in [21]. The corresponding delay model considers gate to drain coupling capacitance 
and short circuit current, and are unnecessarily complicated. The delay model is in [31] for inverter also considers channel length modulation but is also complex because of considering gate to drain coupling capacitance and sub-threshold current.

There are very few models for delay variation. An analytical model for delay variation is derived in [6] where the nominal delay equations are based the $\alpha$-power model. Here gates with stacked transistors are simplified to equivalent inverters, so variation because of different inputs cannot be characterized. There is another piece of work [20] that characterizes delay variations, but no analytical equations are derived to model the variation.

\subsection{Contributions}

The objective of this thesis is to develop an accurate analytical model for predicting nominal delay and delay variability for scaled technologies. First, nominal delay model for inverter is developed at $45 \mathrm{~nm}$ technology node. The model is developed based on accurate current equations that take channel length modulation into consideration. All the factors affecting delay, namely, transistor widths, load capacitance $\left(C_{L}\right)$ and input slew rate $\left(t_{r}\right)$ are considered in the model. The analytical model matches with the HSPICE simulated results closely for both high to low $(\mathrm{HL})$ and low to high( $\mathrm{LH})$ delays. The inverter delay model is applied to $32 \mathrm{~nm}$ technology and here too the model shows very good agreement with HSPICE simulated results.

The nominal delay model is then extended to consider effect of stacked transistors in NAND and NOR gates. It is observed that the delay depends on the position of the transistor with switching input. Specifically transistors in between transistor with switching input and output node contribute to delay, while transistors in between transistor with switching input and supply nodes do not have any effect. This feature is taken into account while deriving a model for nominal delay for gates with stacked transistors. The proposed model is very accurate and matches HSPICE simulated results for NAND and NOR gates at 45nm technology node.

Next, delay variation because of variations in threshold voltage $\left(V_{t h}\right)$ is analyzed. The proposed model for delay variation not only considers $V_{t h}$ variation, but also its dependency on other factors such as $C_{L}$ and $t_{r}$. The variability model is extended to NAND and NOR gates and the variation in each transistor is analyzed separately. The variability model for inverter, NAND and NOR gates closely matches HSPICE simulated results. 
The nominal delay model and variability model are then applied to complex gates like XOR and Full Adder and the results are compared with HSPICE simulated results. Finally, the model is applied to complex ISCAS' 85 benchmark circuits and the results are compared with Synopsys primetime estimated values using 45nm Nangate library [3]. The number of gates in critical paths range from 12-124 in these benchmark circuits. For these critical paths, estimated nominal delay values matches the Synopsys predicted delay within 4\% error. The variability in delay is also predicted for these circuits. The predicted $\sigma / \mu$ for all the critical paths is less than $3 \%$ when $V_{t h}$ variation of $50 \mathrm{mV}$ is given for transistor of width twice minimum length at $45 \mathrm{~nm}$ technology. With varying $\sigma_{V_{t h}}$ some important trends are demonstrated regarding setup and hold times. It is observed that non-critical paths would become critical and rate of hold violations increases enormously with increasing $\sigma_{V_{t h}}$.

\subsection{Thesis Organization}

Chapter 2 discusses the variability trends with CMOS technology scaling. Variability is analyzed for varying path lengths, logic implementation style and sizing based on logical effort. Chapter 3 gives the derivation of nominal delay for inverter and its extension to NAND and NOR gates. The model is verified with HSPICE results. Chapter 4 derives the delay variability equations due to threshold voltage variations for inverter NAND and NOR gates. The model estimated results match the HSPICE simulated results quite closely. The proposed model is verified for XOR2 and Full Adder circuits at 45nm technology in Chapter 5. The model is also used to estimate the delay of complex ISCAS' 85 benchmark circuits and the results are compared with Synopsys ptimetime estimated values using $45 \mathrm{~nm}$ library [3]. The use of the proposed model into the design flow is also demonstrated. It is shown how possible timing errors due to variability can be easily identified. Chapter 6 concludes the work. 


\section{Chapter 2}

\section{VARIABILITY AND RELIABILITY ANALYSIS}

Variability in circuit performance increases with technology scaling because of increasing threshold voltage variations. This chapter begins with the classification of variation and causes of variation in threshold voltage of transistors (Section 2.1). This is followed by an analysis of variability trends with technology scaling in gates like inverter, AND6 and circuits like inverter chain, 6-T SRAM (Section 2.2). A mechanism to reduce variability by increasing the length of transistor is also presented here. Variability dependency on factors like path length (Section 2.3), differences in implementations of the same logic function (Section 2.4) and logical effort sizing are also studied (Section 2.5).

\subsection{Background}

CMOS scaling is advancing towards the $10 \mathrm{~nm}$ regime [2]. Such aggressive scaling inevitably leads to vastly increased variability in circuit performance, posing a grand challenge to future robust IC design.

Classification of variations: Threshold voltage variations in CMOS can be divided into interdie variations and intra-die variations. Inter-die variations are systematic variations and affect adjacent transistors on a chip with equal shift from nominal value. Intra-die variations are random variations and affect adjacent transistors on same chip with different shifts. Inter-die variations can be adjusted by adapting the supply voltage, $V_{D D}$ to compensate for shifted threshold voltage [15]. Forward and reverse-body bias techniques can also be used [9, 17, 15] to compensate for inter-die variations. Inter-die variations affect variability in combinational circuits more than sequential circuits.

Intra-die variations are more difficult to solve because these variations are not systematic. However the variations reduce because of averaging effect with increasing path length. In conventional circuit design techniques, transistors in non-critical paths are replaced with high$V_{t h}$ transistors to reduce leakage power. But this technique increases the delay of non-critical paths and these non-critical paths can become critical paths because of $V_{t h}$ variation. Hence conventional design techniques to reduce power cannot be directly applied to future technology designs as power and variability pose opposite constraints [18]. 


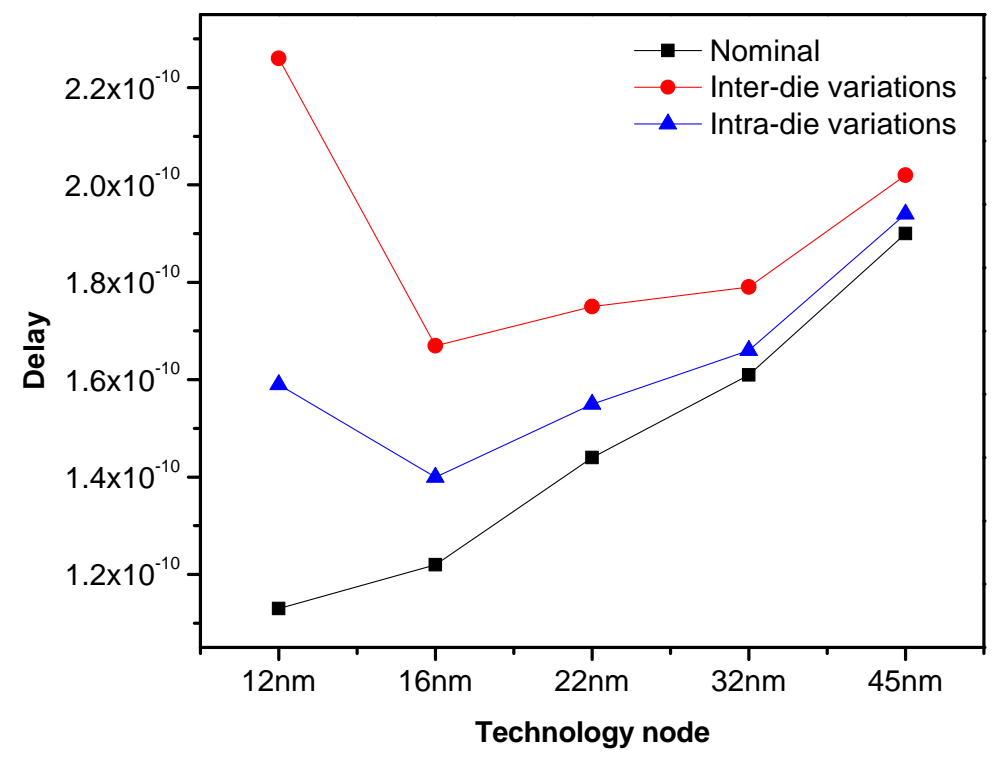

Figure 2.1: Effect of inter-die and intra-die variations in NAND2-RO delay, at different technologies.

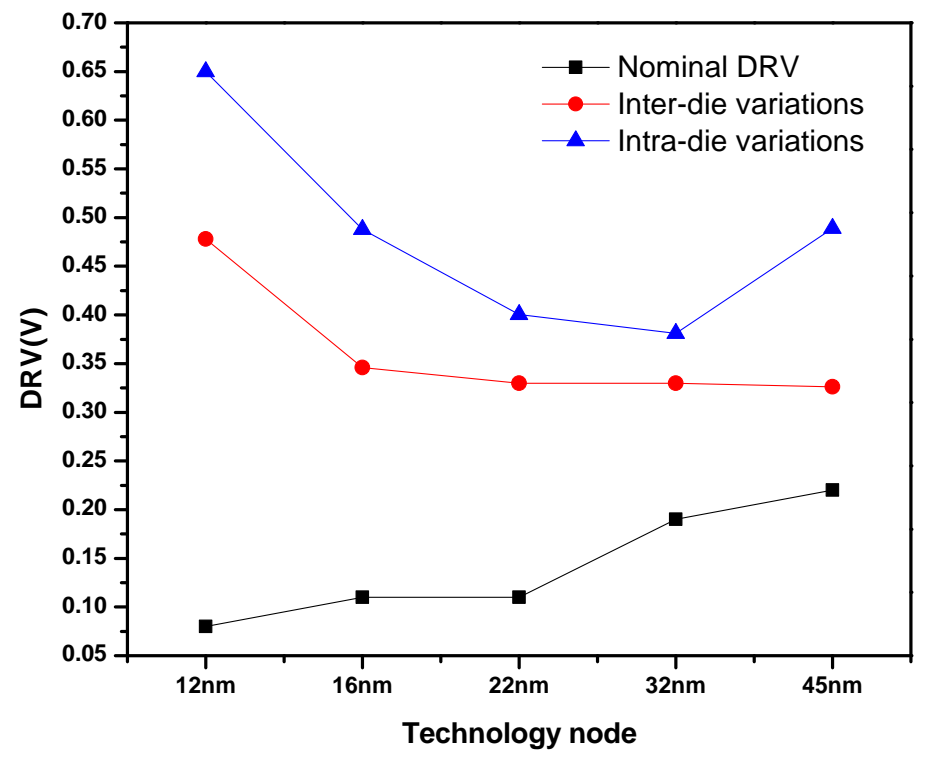

Figure 2.2: Effect of inter-die and intra-die variations in 6T-SRAM DRV, at different technologies. 
To analyze the effect of inter-die and intra-die variations, we consider the 11-NAND2 Ring Oscillator(RO) and 6-T SRAM circuits. Figure 2.1 shows the shift in delay of NAND2-RO at different technology nodes and Figure 2.2 shows the shift in Data Retention Voltage(DRV) of the SRAM circuit at different technology nodes. In NAND2-RO, random variations tend to average out and result in smaller variability than systematic variations for all technologies. In SRAM circuit, mismatch in threshold voltages of transistors because of random intra-die variations causes more shift in Data Retention Voltage than systematic inter-die variations. Thus intra-die variations affect sequential circuits more than combinational circuits.

Sources of Variations: Variations are caused by intrinsic variations and manufacturing-induced variations. The manufacturing induced variations arise from imperfections in the fabrication process, and vary from foundry to foundry. Moreover, they exhibit a strong dependence on layout patterns, such as layout-dependent stress effect. These variations could be reduced by a better control of the process. On the other hand, intrinsic variations are limited by fundamental physics. They are inherent to CMOS structure and considered as one of the ultimate bottlenecks to CMOS scaling. The intrinsic variations are primarily due to random dopant fluctuation, lineedge roughness and oxide thickness fluctuation. These fundamental variation sources cause a shift in the values of device parameters, especially $V_{t h}$, and result in significant variations in the performance of a scaled device. Their influence keeps increasing with the reduction of CMOS feature size as will be demonstrated in Section 2.2.

Random dopant fluctuation (RDF) is caused by random placement of dopant atoms in the channel region. During dopant implantation [33], there exists some randomness in the amount of and position of dopants, resulting in fluctuation of total number of dopants and hence the $V_{t h}$ value. As the device size scales down, the total number of channel dopants decreases and such a decrease results in an dramatic increase in threshold variation [29]. Fluctuation in dopant number usually follows a Poisson distribution [19]. If there are enough dopants in the channel region, the distribution of total number of dopants can be approximated as a Gaussian distribution [22].

Line edge roughness (LER) is the distortion of gate shape along channel width direc- 
tion [33]. This variation is mainly affected by the process of gate etching, and is inherent to gate materials $[23,7,14,12]$. The concerning fact is that LER variation does not scale with technology and the improvement in the lithography process does not effectively reduce such an intrinsic variation. Numerical simulations and silicon data further indicate that the LER effect significantly increases the leakage and threshold variations.

Oxide Thickness Fluctuation (OTF) is caused by the atom scale surface roughness of the $\mathrm{Si}-\mathrm{SiO}_{2}$ and $\mathrm{Gate}_{-} \mathrm{SiO}_{2}$ interfaces [8]. When oxide thickness is equivalent to only a few silicon atom layers, the atomic scale interface roughness steps result in significant oxide thickness variation [16]. The unique random pattern of the gate oxide thickness and interface landscape makes each MOSFET different from its counterparts and leads to variations in the surface roughness. This affects mobility, gate tunnelling current $[30,11]$ and hence threshold voltage variation from device to device.

\subsection{Variability in Circuit Performance}

The variations in $V_{t h}$ are applied to two benchmark circuits - inverter chain and 6T-SRAM, and the variability in their performance is quantified. For the inverter chain, the performance metric is chosen to be delay and for 6T SRAM, the performance metrics are read access time and read noise margin (RNM).

\subsubsection{Case Study - Inverter}

An inverter chain is built with seven inverters as shown in Figure 2.3. Delay measurements are made across the fourth inverter, because it is isolated from both input and output loading effects [2]. Length of both PMOS and NMOS is kept at the minimum feature size of the specific technology. Width of NMOS is taken to be 8 times the minimum length. Width of PMOS is found so that rise and fall times are equal. Table 2.1 shows $p-n$ ratios for technologies from $45 \mathrm{~nm}$ to $12 \mathrm{~nm}$. These are the $p-n$ ratios used throughout this work.

100 Monte Carlo simulations are run by adding independent variation in $V_{t h}$ for all fourteen transistors in the inverter chain. Figure 2.4 shows the trend in nominal delay and delay variations with technology scaling. As seen from the Figure 2.4, delay decreases with scaling but variability, as a percentage of mean, increases rapidly because of increasing $V_{t h}$ 


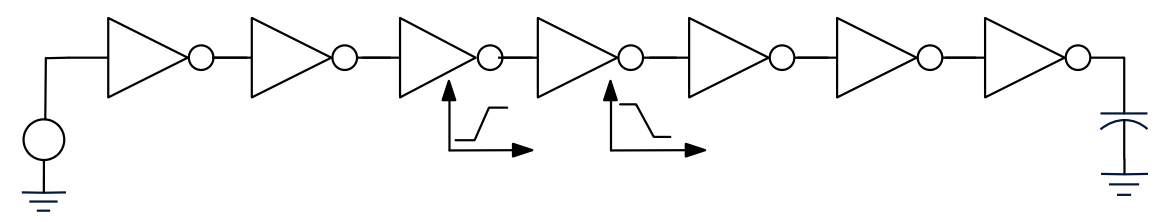

Figure 2.3: Schematic of 7-inverter chain.

\begin{tabular}{|c|c|c|c|}
\hline Technology & $\mathrm{L}(\mathrm{nm})$ & $V_{D D}(\mathrm{~V})$ & p-n ratio \\
\hline $45 \mathrm{~nm}$ & 45 & 1.0 & 1.02 \\
\hline $32 \mathrm{~nm}$ & 32 & 0.9 & 0.96 \\
\hline $22 \mathrm{~nm}$ & 22 & 0.8 & 0.91 \\
\hline $16 \mathrm{~nm}$ & 16 & 0.7 & 0.80 \\
\hline $12 \mathrm{~nm}$ & 12 & 0.65 & 0.84 \\
\hline
\end{tabular}

Table 2.1: Minimum length, $V_{D D}$, and p-n ratios of inverter for different technologies.

variation. This implies that technology scaling assures improved nominal performance but when variability is considered, it degrades the robustness of the circuit.

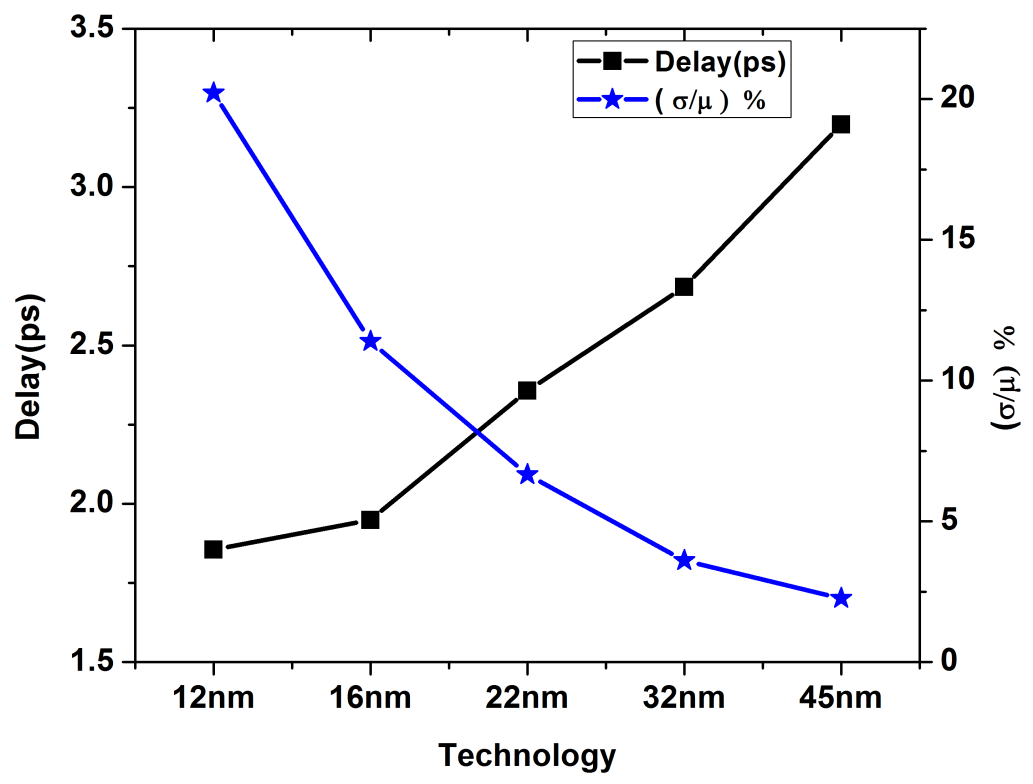

Figure 2.4: Inverter: Mean delay and sigma as percentage of mean delay.

Further, the contribution of individual intrinsic factors, namely, RDF, LER and OTF, towards delay is analyzed. This is done by applying $V_{t h}$ variation because of each of these factors to the inverter circuit. The variation in $V_{t h}$ is calculated using the method in [32] . Figure 2.5 illustrates the contribution of RDF, LER and OTF for different technology nodes. LER and OTF 
are the major contributors to variability in lower technology nodes. The impact of LER on $V_{t h}$ variation is mainly because of fluctuation of channel length in the gate width direction, which is also called gate line-width roughness (LWR) [24]. The channel length fluctuation combined with severe short channel effect contributes to a large $V_{t h}$ variation. OTF causes the fluctuation of gate voltage drop across oxide layer, and further results in $V_{t h}$ variation. This effect becomes pronounced during scaling because height of the atomic layer at oxide surface does not scale with the oxide thickness. Therefore, the average fluctuation becomes larger as the area of gate oxide scales.

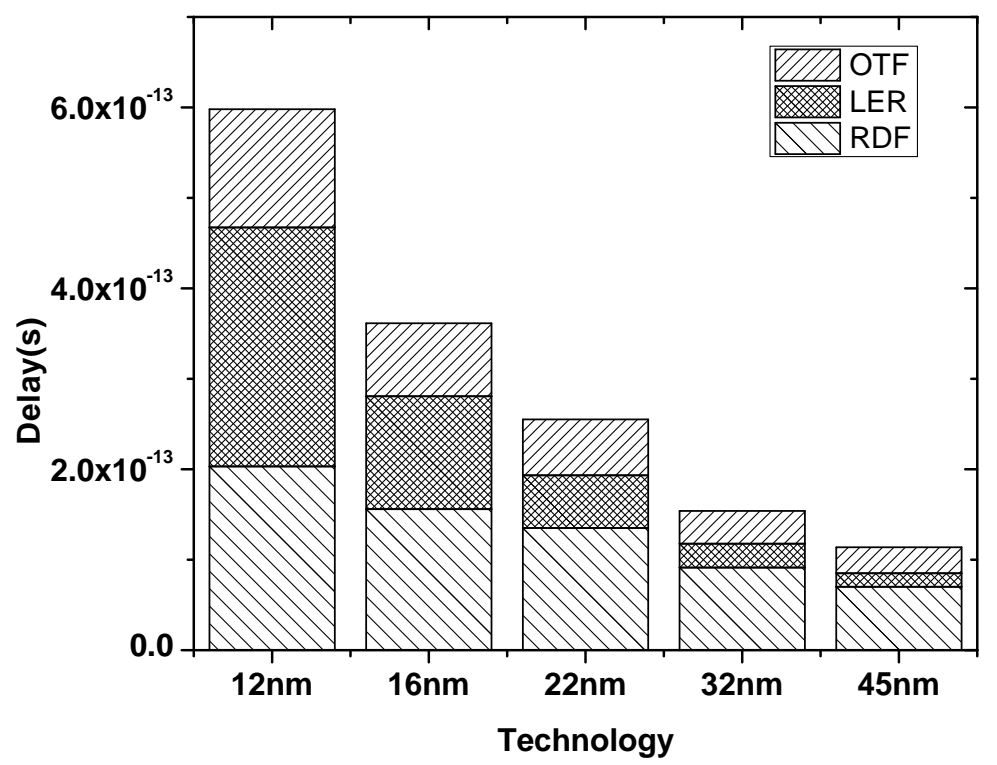

Figure 2.5: Inverter delay variation due to each intrinsic factor.

\subsubsection{Case Study - 6T-SRAM}

The effect of $V_{t h}$ variation on Read Access time and RNM are examined for a typical 6T-SRAM cell shown in Figure 2.6. All the six transistors have minimum length for simplicity. The pull up PMOS transistors are assigned minimum width. The widths of access transistor and pull down NMOS are found by making the read and write noise margins equal [13]. The transistor widths for all the technologies are given in Table 2.2.

Read Access Time: Read Access Time depends on sense amplifiers at the output of SRAM circuit. Assuming that sense amplifiers are able to measure $10 \%$ of $V_{D D}$ drop on either 


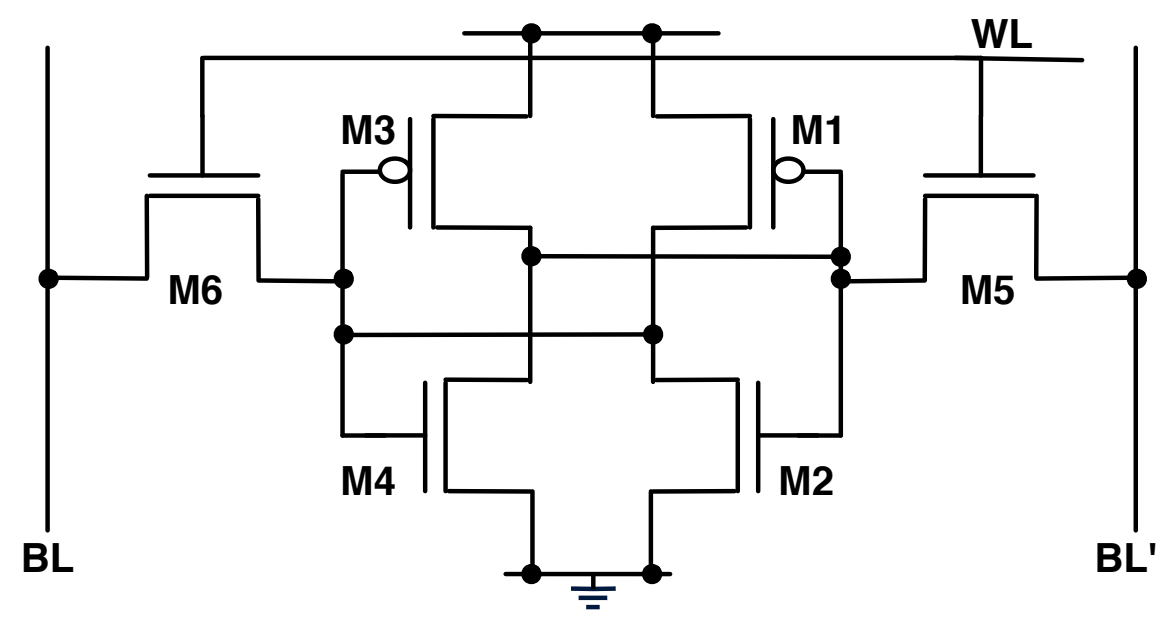

Figure 2.6: Schematic of 6T-SRAM circuit.

\begin{tabular}{|c|c|c|c|}
\hline Technology & Pullup PMOS(nm) & Pulldown NMOS(nm) & access transistor(nm) \\
\hline $45 \mathrm{~nm}$ & 45 & 45 & 45 \\
\hline $32 \mathrm{~nm}$ & 32 & 32 & 32 \\
\hline $22 \mathrm{~nm}$ & 22 & 22 & 22 \\
\hline $16 \mathrm{~nm}$ & 16 & 16 & 24 \\
\hline $12 \mathrm{~nm}$ & 12 & 12 & 24 \\
\hline
\end{tabular}

Table 2.2: SRAM transistor widths when length is taken to be minimum.

bitline (BL) or its complement (BL'), read access time is calculated as the time BL or BL' drops to $90 \%$ of $V_{D D} . V_{t h}$ of the transistors is varied independently and Monte Carlo simulations are performed. $V_{t h}$ is assumed to follow Gaussian distribution, so the access time variation should also follow Gaussian distribution. The mean and standard deviation of access time are plotted for three technologies $45 \mathrm{~nm}, 22 \mathrm{~nm}$ and $12 \mathrm{~nm}$ as shown in Figure 2.7. The ratio of standard deviation and mean of access time is $2 \%$ at $45 \mathrm{~nm}$ and $41 \%$ at $12 \mathrm{~nm}$. The variation at $12 \mathrm{~nm}$ is clearly unacceptably large.

Reducing Variability by Increasing Device Length: The increase in performance variation for lower technology nodes is due to increase in $V_{t h}$ variation because of LER and OTF. Both LER and OTF effects are because of small geometry of device and reduce significantly with slight increase in physical device size. Increasing length of device causes increase in access time at nominal $V_{t h}$. A study is done to see if smaller variation in $V_{t h}$, because of increased length, will reduce worst case access time calculated by $\mu+3 \sigma$. Monte Carlo simulations are repeated for the case when the gate length $(\mathrm{L})$ is increased by $10 \%$. The ratio of standard deviation to mean 


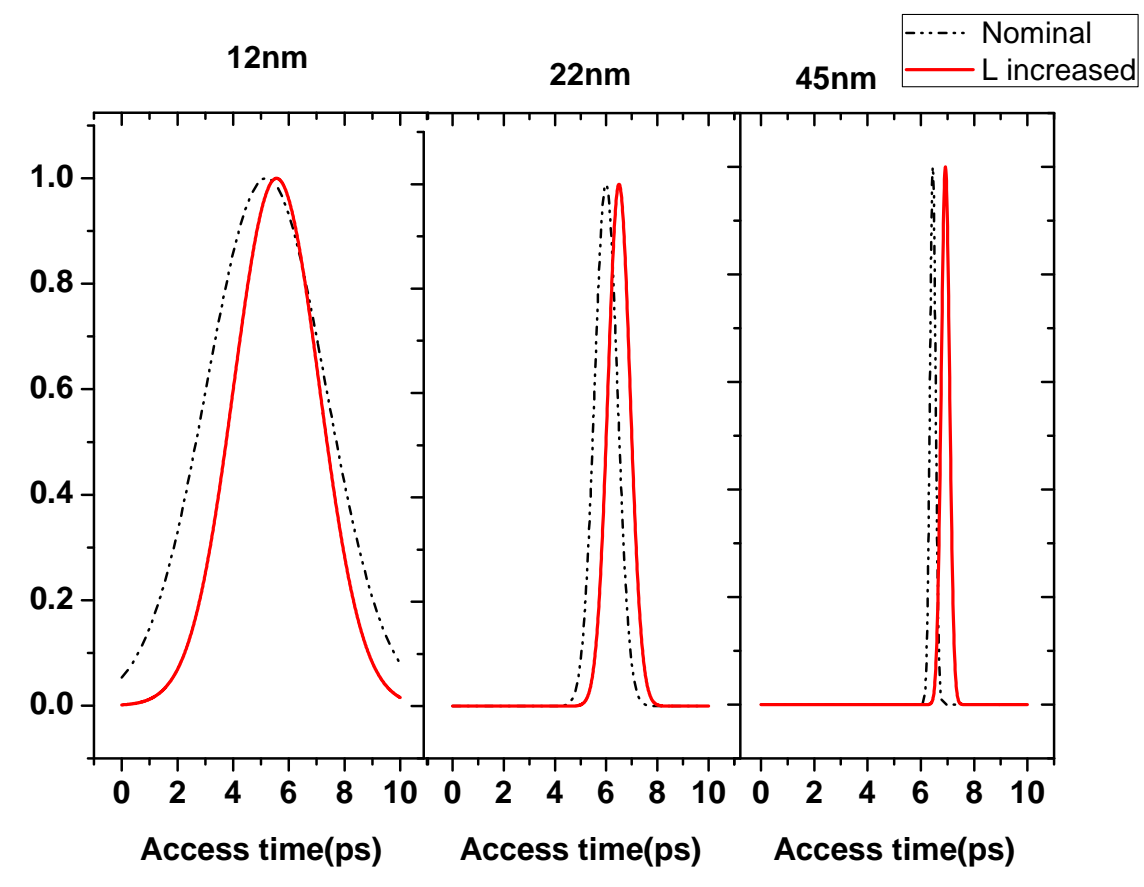

Figure 2.7: SRAM: Comparison of access time PDF's in 45nm, 22nm, and 12nm technologies.

of access time drops to $28 \%$ at $12 \mathrm{~nm}$ at the cost of slight reduction in nominal performance!

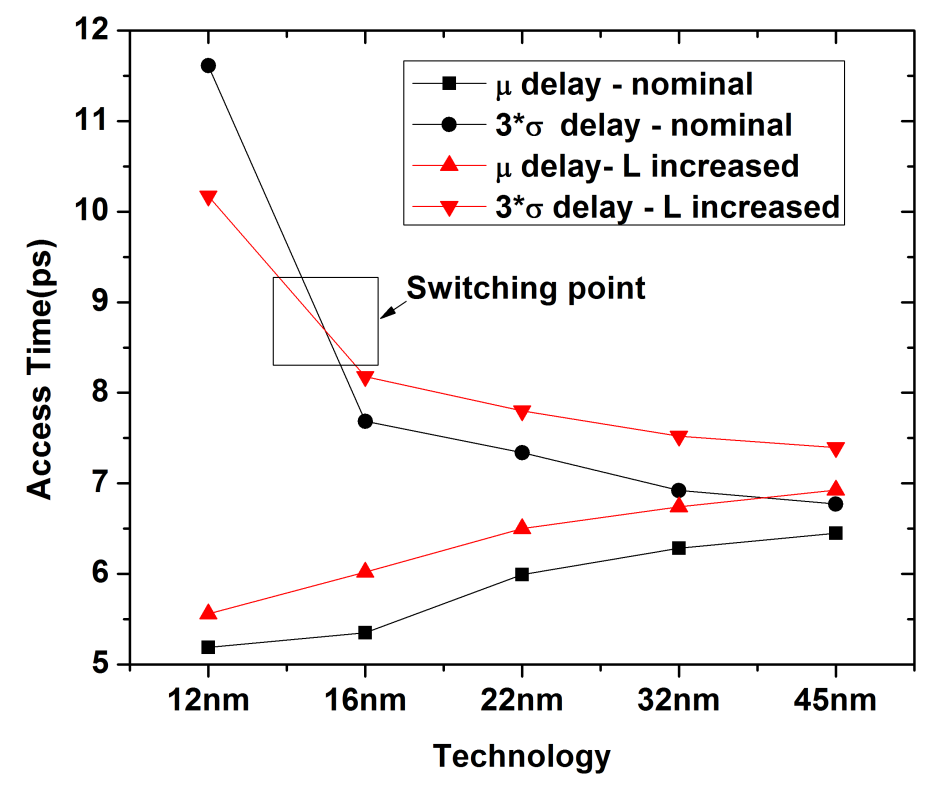

Figure 2.8: SRAM. Mean and $3 \sigma$ point for all technologies 
The simulation results show that at $45 \mathrm{~nm}$ technology node, the performance variation does not improve with increase in L. At $22 \mathrm{~nm}$, the variation decreases but is not large enough to bring the $3 \sigma$ access time less than the nominal. However at $12 \mathrm{~nm}$, the $3 \sigma$ access time with $10 \%$ larger $\mathrm{L}$ is less than the nominal case. Figure 2.8 also shows that with scaling, while the nominal access time reduces, the worst case delay increases. By tuning gate length to $10 \%$ more than nominal, the access time increases for each technology but the trend with technology scaling remains the same. The worst case delay at $12 \mathrm{~nm}$ reduces below the nominal thus giving tightly coupled performance variation than at nominal. As variation in performance is small in current technologies, the focus of process tuning should be to enhance the nominal performance but with scaling, variability becomes an important parameter.

Read Noise Margin $(R N M)$ : For a first order analysis, RNM is considered to be a linear function of mismatches between $V_{t h}$ of transistors. The following six mismatches are considered and all are taken to be independent.

1. Mismatch between $M 1, M 2$ and between $M 3, M 4$

2. Mismatch between $M 1, M 3$ and between $M 2, M 4$

3. Mismatch between $M 2, M 5$ and between $M 4, M 6$

The variations in $V_{t h}$ of each transistor are directly mapped to mismatches between pairs as listed above. The variation of mismatch is considered to be summation of variation of both transistors as given in equation below.

$$
\sigma_{V_{t h,(M 1 M 2)}}^{2}=\sigma_{V_{t h,(M 1)}}^{2}+\sigma_{V_{t h,(M 2)}}^{2}
$$

Threshold voltage of each transistor is changed separately and shift in RNM is observed. The $\beta$ coefficients are calculated empirically from linear equation between mismatch and RNM. The variation in RNM is calculated from variation of mismatches and $\beta$ coefficients as given in:

$$
\begin{aligned}
\sigma_{R N M}^{2}= & \beta_{1}^{2} \sigma_{V_{t h,(M 1 M 2)}^{2}}^{2}+\beta_{2}^{2} \sigma_{V_{t h,(M 3 M 4)}}^{2}+\beta_{3}^{2} \sigma_{V_{t h,(M 1 M 3)}}^{2} \\
& +\beta_{4}^{2} \sigma_{V_{t h,(M 2 M 4)}^{2}}^{2}+\beta_{5}^{2} \sigma_{V_{t h,(M 2 M 5)}}^{2}+\beta_{6}^{2} \sigma_{V_{t h,(M 4 M G)}}^{2}
\end{aligned}
$$


The variation due to the individual intrinsic parameters is calculated and is shown in Figure 2.9. Similar to inverter delay variation, the contribution of LER and OTF on SRAM RNM variation increases with technology scaling. The variation due to RDF dominates till $22 \mathrm{~nm}$, but below that, LER and OTF are the major contributors. The mean of RNM for $12 \mathrm{~nm}$ is $0.074 \mathrm{~V}$ and from Figure 2.9 we can see that variability is very large. SRAM is very sensitive to mismatches and its sensitivity increases significantly with scaling.

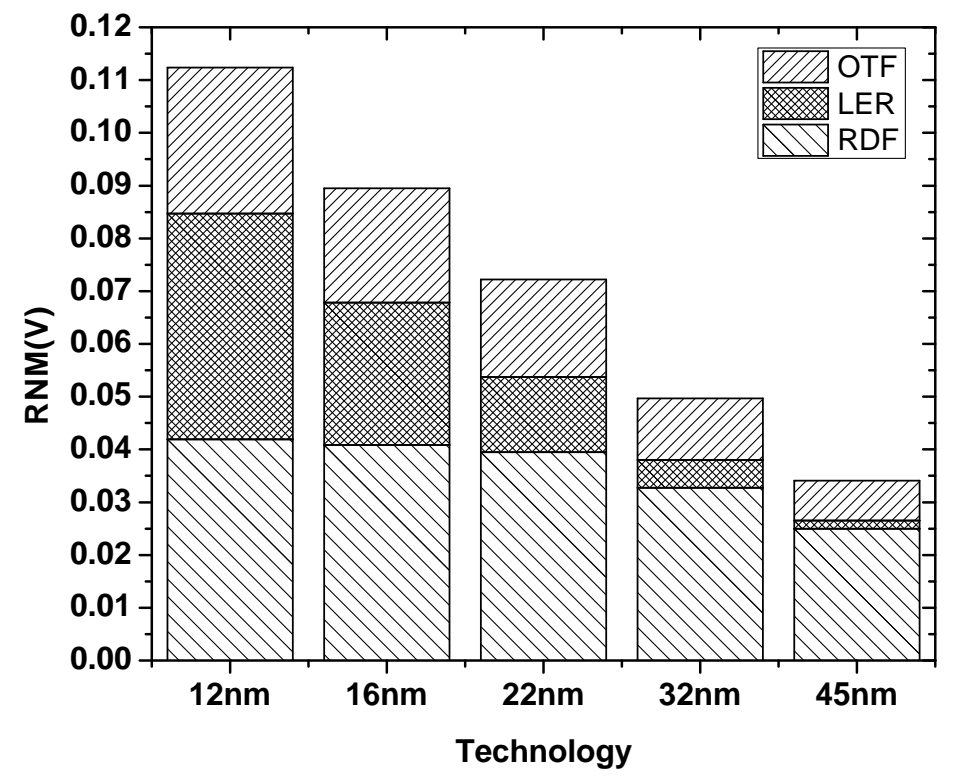

Figure 2.9: SRAM RNM variability due to each intrinsic factor variation.

\subsection{Effect of Variability on Path length}

To evaluate variability of circuits, with different path lengths, a ring oscillator with 51 inverters is considered. Delay across different number of gates is observed. As path length increases, nominal delay increases in proportion to the number of gates in the path. Variation in threshold voltage is considered to be Gaussian distributed and completely independent in each transistor. So variation in delay is given by equation (2.3) [28] and increases with $\sqrt{N}$, where $\mathrm{N}$ is the number of stages.

$$
\sigma_{\text {path }}=\sqrt{\sum \sigma_{\text {gate }}^{2}}
$$




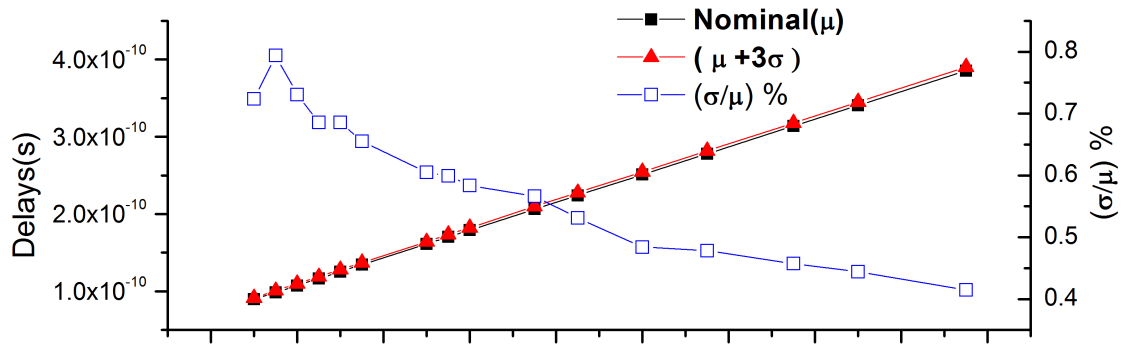

(a)

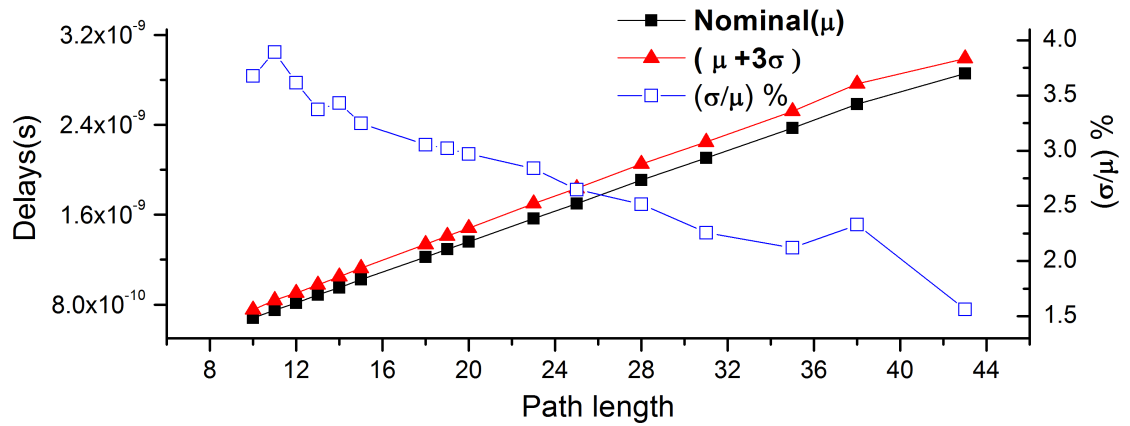

(b)

Figure 2.10: Nominal Delay and Delay variation with different path lengths at $45 \mathrm{~nm}$ technology node at (a) nominal voltage of $V_{D D}=1.0 \mathrm{~V}$, (b) $V_{D D}=0.5 \mathrm{~V}$.

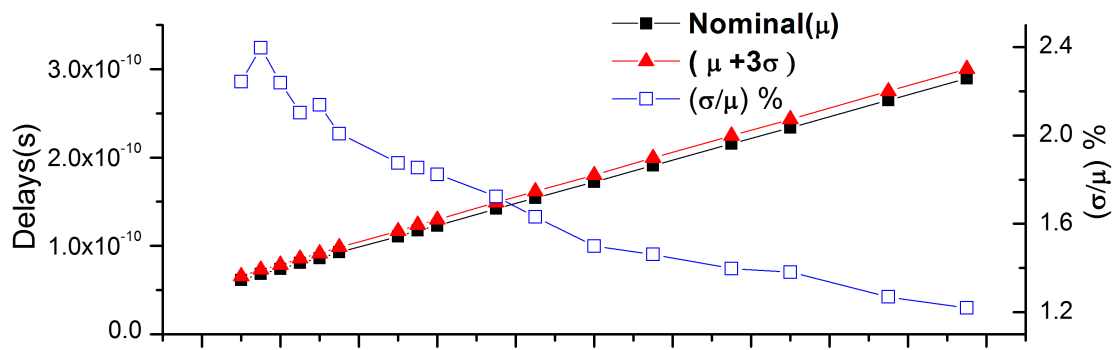

(a)

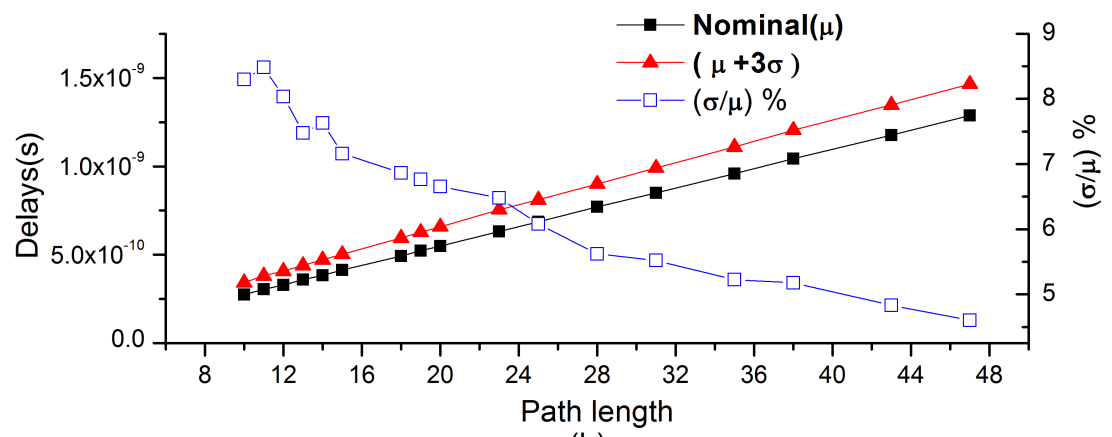

(b)

Figure 2.11: Nominal Delay and Delay variation with different path lengths at 22nm technology at (a) nominal voltage of $V_{D D}=0.8 \mathrm{~V}$, (b) $V_{D D}=0.5 \mathrm{~V}$. 


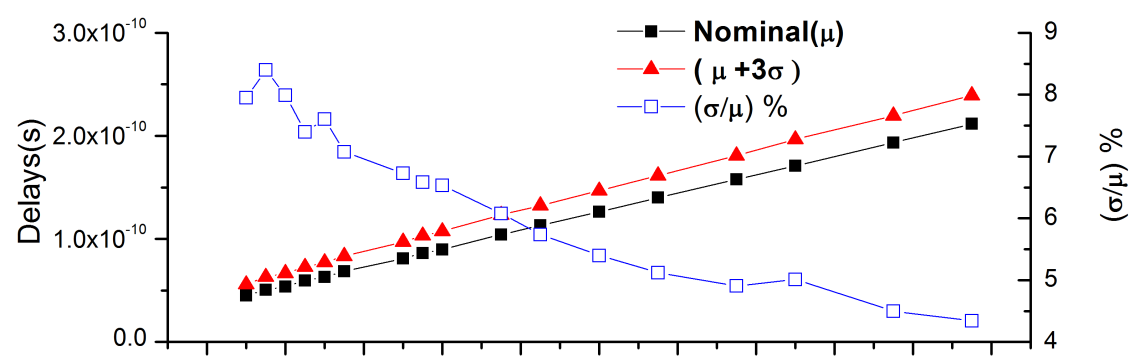

(a)

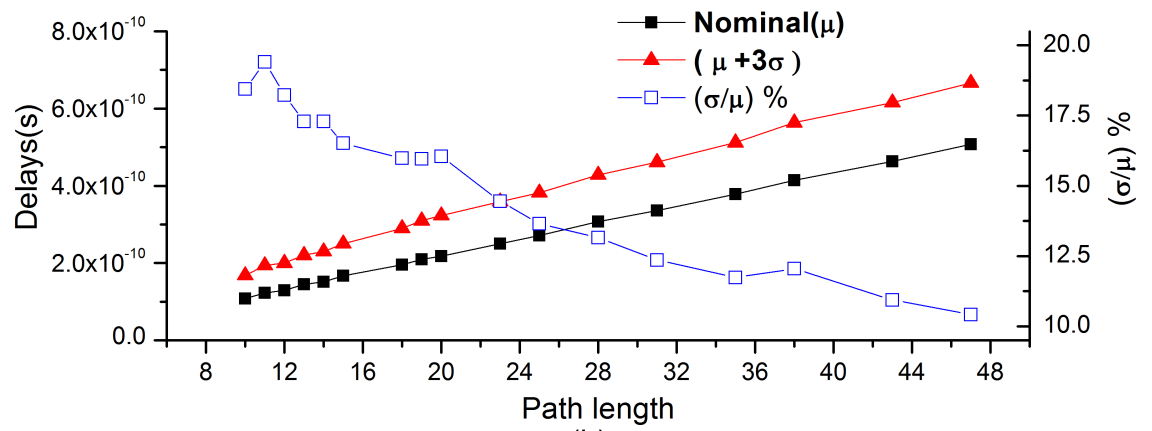

(b)

Figure 2.12: Nominal Delay and Delay variation with different path lengths at $12 \mathrm{~nm}$ technology at (a) nominal voltage of $V_{D D}=0.65 \mathrm{~V}$, (b) $V_{D D}=0.5 \mathrm{~V}$

So overall, the worst case delay, $\mu+3 \sigma$ keeps increasing with increasing path length. But the variation in delay as a percentage of nominal delay, $\sigma / \mu$, keeps decreasing. Figures 2.10 - 2.12 show these trends with increasing path length. Figure 2.10a shows nominal delay, worst case delay $(\mu+3 \sigma)$ and $\sigma / \mu$ of inverter chain at $45 \mathrm{~nm}$ technology node at nominal voltage of $V_{D D}=1.0 \mathrm{~V}$. Figure $2.10 \mathrm{~b}$ plots the same at $V_{D D}=0.5 \mathrm{~V}$. Nominal delay for path length of 31 inverters is $277.9 \mathrm{ps}$ at nominal $V_{D D}=1.0 \mathrm{~V}$ and $2104 \mathrm{ps}$ at $V_{D D}=0.5 \mathrm{~V}$. Delay variation $(\sigma)$ because of threshold voltage variation calculated using the method in [32] is $1.329 \mathrm{ps}$ at nominal $V_{D D}$ and $47.5 \mathrm{ps}$ at $V_{D D}=0.5 \mathrm{~V}$. Thus variability $(\sigma / \mu \%)$ increased from $0.47 \%$ at nominal $V_{D D}$ to $2.25 \%$ at lower $V_{D D}$. This shows that variability becomes increasingly important for low power applications, where supply voltage is reduced. Similar trends are observed for $22 \mathrm{~nm}, 12 \mathrm{~nm}$ at nominal voltage and at $V_{D D}=0.5 \mathrm{~V}$ as shown in Figures 2.11 and 2.12.

As both nominal delay and delay variation increase with increasing number of gates in a path, worst case delay $(\mu+3 \sigma)$ also keeps increasing. This trend can be clearly seen at 12nm technology node in Figure 2.12. At 12nm technology node, for nominal supply voltage, 
when path length is 10 , the difference between nominal and worst case delay curves (that is $3 \sigma$ ) is $10.74 \mathrm{ps}$ and this difference increases to $27.55 \mathrm{ps}$ at path length of 47 . Hence even though random variations average out with increasing path length, path length cannot be increased to reduce delay variation. But the delay variation with respect to nominal delay $(\sigma / \mu \%)$ becomes small with increasing path length. It reduces from $7.94 \%$ for path length of 10 inverters to $4.33 \%$ for path length of 47 at $12 \mathrm{~nm}$ technology node.

Threshold voltage variations across technology nodes keeps increasing with technology scaling. While delay variation increases, the nominal delay decreases and $\sigma / \mu$ increases significantly. At nominal voltages, $\sigma / \mu$ at $45 \mathrm{~nm}$ for path length of 43 inverters is $0.41 \%$, while it is $1.27 \%$ at $22 \mathrm{~nm}$ and $4.5 \%$ at $12 \mathrm{~nm}$. Further $V_{D D}$ scaling at $22 \mathrm{~nm}$ and $12 \mathrm{~nm}$ technologies increases $\sigma / \mu$ to $4.8 \%$ at $22 \mathrm{~nm}$ and $10.93 \% 12 \mathrm{~nm}$. Such high values for even large path lengths makes these circuits unreliable at scaled technologies.

\subsection{Effect of Variation on Logic Style}

Any logic function can be implemented in multiple ways. Figure 2.13 shows how large gates like AND6 can be implemented in multiple ways. We study how variation may be affected by the way a function is implemented using AND6 as an example. The first implementation has 6 NMOS transistors stacked, so width of NMOS is $6 W_{n}$. The second implementation has 3 transistors stacked, so width is $3 W_{n}$. The third implementation has only two transistors in stack and width is $2 W_{n}$. The final implementation has 3 stacked trnansistors and width is $3 W_{n}$. Switching input in a stack is always given to the transistor farthest from output so that maximum delay in the gate is considered. Low to high delay is considered as performance metric because this triggers the stack in both NAND and NOR gates.

Variation in $V_{t h}$ is smallest in the first implementation, because $\sigma_{V_{t h}} \propto \frac{1}{\sqrt{W}}$ and the first implementation has the widest gate. Variation in delay relative to nominal delay is small in the first case when compared to second and third implementations. But nominal delay value is high for that implementation because of large stack. So worst case delay, $\mu+3 \sigma$ is large for first case compared with all other implementations. For the fourth implementation, nominal delay is large because of multiple stages but $\sigma / \mu$ is smaller than second and third implementations because multiple stages average out the effect of random variations. Both second and third 

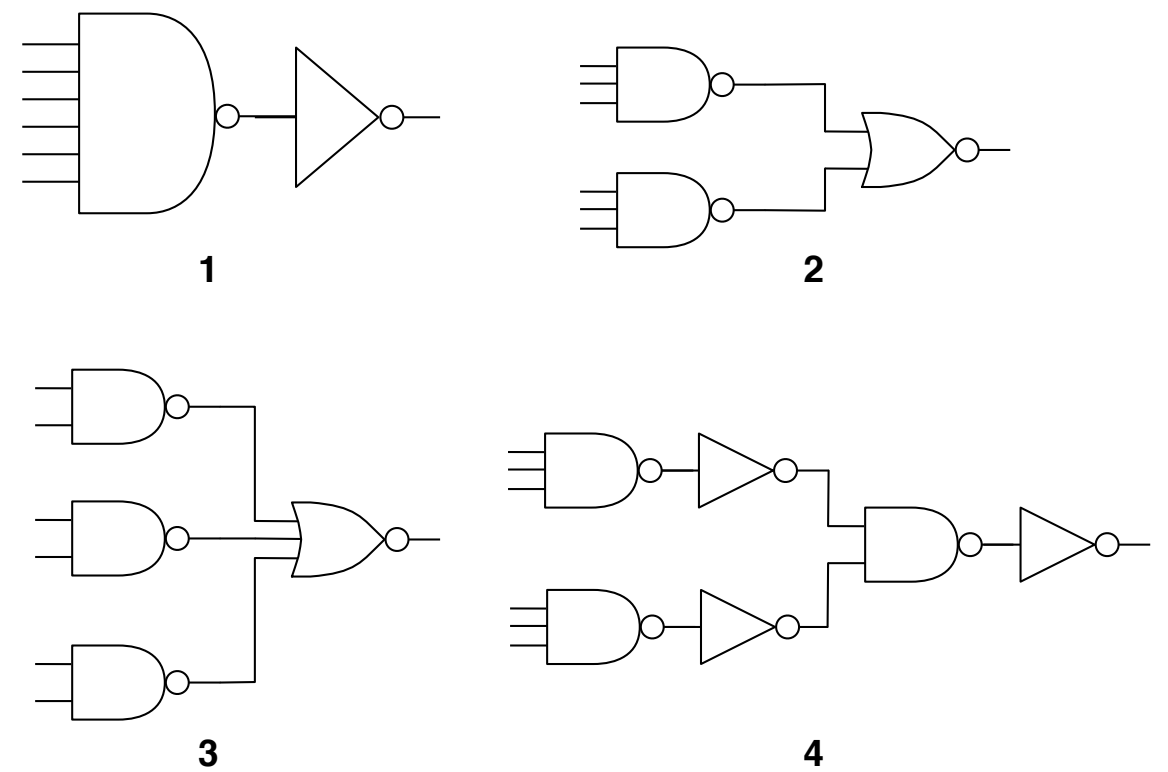

Figure 2.13: Different implementations of AND6 function.

implementations give almost the same delay and delay variability because both have similar stacks and it is not clear as to which is better circuit.

The delay and delay variation results for $45 \mathrm{~nm}$ technology are summarized in Table 2.3. Table 2.4 shows similar trends for $12 \mathrm{~nm}$ technology. So in all the implementations, circuits with lower nominal delay gives lower variability. This is because variability depends on nominal value along with amount of threshold voltage variation. This can be shown as follows. Delay $T_{p} \propto \frac{1}{I_{D}}$, and $I_{D} \propto\left(V_{D D}-V_{t h}\right)$. From [28], we have

$$
\sigma_{T_{p}}=\frac{\partial\left(T_{p h l}\right)}{\partial V_{t h}} \sigma_{V_{t h}}
$$

Substituting we get,

$$
\sigma_{T_{P}}=\sigma_{V_{t h}} \frac{\partial T_{P}}{\partial V_{t h}}=\sigma_{V_{t h}} \frac{T_{P}}{V_{D D}-V_{t h}}
$$

\begin{tabular}{|c|c|c|c|c|}
\hline Implementation & $\mu(\mathrm{ps})$ & $\sigma(\mathrm{ps})$ & $\mu+3 \sigma(\mathrm{ps})$ & $\sigma / \mu \%$ \\
\hline 1 & 18.96 & 0.19 & 19.54 & 1.03 \\
\hline 2 & 11.72 & 0.13 & 12.11 & 1.11 \\
\hline 3 & 12.01 & 0.13 & 12.40 & 1.08 \\
\hline 4 & 18.33 & 0.18 & 18.87 & 0.98 \\
\hline
\end{tabular}

Table 2.3: Nominal delay and delay variation when AND6 is implemented in different styles at $45 \mathrm{~nm}$ technology node. 


\begin{tabular}{|c|c|c|c|c|}
\hline Implementation & $\mu(\mathrm{ps})$ & $\sigma(\mathrm{ps})$ & $\mu+3 \sigma(\mathrm{ps})$ & $\sigma / \mu \%$ \\
\hline 1 & 18.76 & 1.18 & 22.30 & 6.29 \\
\hline 2 & 10.04 & 0.84 & 12.56 & 8.37 \\
\hline 3 & 9.50 & 0.90 & 12.19 & 9.42 \\
\hline 4 & 13.98 & 1.16 & 17.46 & 8.30 \\
\hline
\end{tabular}

Table 2.4: Nominal delay and delay variation when AND6 is implemented in different styles at $12 \mathrm{~nm}$ technology node.

\subsection{Variability and Logical Effort}

A path which is sized according to logical effort can have

- fewer gates with high electrical effort per gate or

- more number of gates with low electrical effort per gate.

Longer paths with slowly increasing gate sizes should have lower variability than small paths with rapidly increasing gate sizes. This is because in both cases, sizes are increasing which decreases variation in $V_{t h}$. But longer paths tend to average out effect of random variations so variation should be less. As an example, consider an inverter chain, loaded with $1 \mathrm{pF}$ capacitance as shown in Figure 2.14. The first stage inverter is fixed to be 8 times minimum size. The buffer stage is designed with different number of stages and gate sizing of each stage is calculated through logical effort. The results are shown in Table 2.5 for $12 \mathrm{~nm}$ technology node.

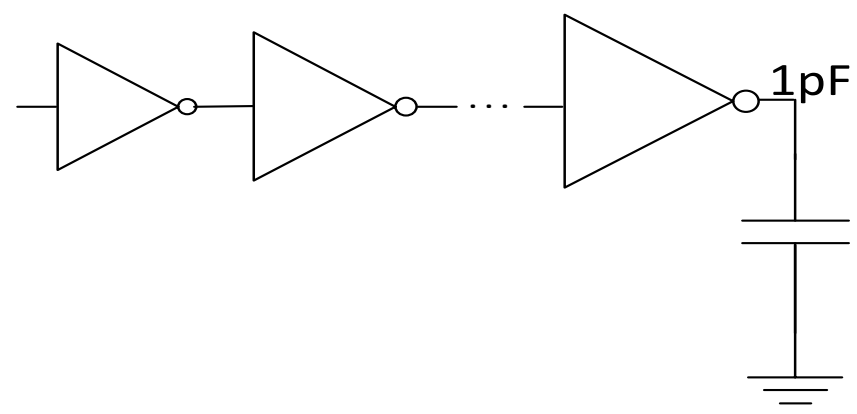

Figure 2.14: Buffer loaded with 1pF capacitance. 


\begin{tabular}{|c|c|c|c|c|}
\hline No. of Stages & Fanout & $\mu(\mathrm{ps})$ & $\sigma(\mathrm{ps})$ & $\mu+3 \sigma(\mathrm{ps})$ \\
\hline 2 & 30 & 34.40 & 3.005 & 43.42 \\
\hline 4 & 5 & 20.40 & 1.362 & 24.49 \\
\hline 4 & 4 & 18.82 & 1.281 & 22.66 \\
\hline 6 & 3 & 27.13 & 1.121 & 30.49 \\
\hline 8 & 2 & 23.69 & 1.237 & 27.40 \\
\hline
\end{tabular}

Table 2.5: Nominal delay and delay variation of buffer stage driving 1pf load with different number of stages at $12 \mathrm{~nm}$ technology node.

From Table 2.5, we see that fanout 4 has the least nominal delay. Variation in delay should decrease when number of stages increases because of averaging out of random variations and also because of increasing gate sizes that decreases $V_{t h}$ variation. But variability depends on nominal delay also and nominal delay increases with increasing number of stages. Because of these opposite trends, the variation for path lengths 4,6 and 8 are almost the same. Increasing nominal delay increases worst case $(\mu+3 \sigma)$ performance for path lengths 4,6 and 8 . For path length of 2 inverters, nominal delay is high because of high load on each inverter and variability is high because it is proportional to nominal delay. So it is best to design circuits with minimum number of gates while keeping the nominal delay low. 


\section{Chapter 3}

\section{ANALYTICAL MODEL FOR NOMINAL DELAY}

An analytical model for nominal delay is developed in this chapter. Delay equations are initially derived for CMOS inverter gate from current equations which consider short channel effects [21] in Section 3.1. The model is quite detailed and accounts for width of gate, loading capacitance and input transition time. The derivation is extended to account for stacked transistors in NAND and NOR gates in Section 3.2. The analytical models are validated using PTM models [4] at 45nm technology and 32nm technology nodes.

\subsection{Nominal Delay Model for Inverter}

The inverter delay models derived with current equations from Shockley's MOSFET model or Sakurai's $\alpha$-power law [27] do not apply as technology scales down below 50nm. This is because channel length modulation becomes important in scaled technologies and saturation current is no longer constant. In fact, saturation current is a function of drain-source voltage $\left(V_{D S}\right)$. The current equation for scaled devices has been derived in [21] and is given below.

$$
I_{D}= \begin{cases}0, & \left(V_{G S} \leq V_{t h}: \text { cutoff }\right), \\ \beta_{l}\left(V_{i n}-V_{t h}\right)^{\alpha} V_{D S}, & \left(V_{D S}<V_{D S A T}: \text { linear }\right), \\ \beta_{S}\left(V_{i n}-V_{t h}\right)^{\alpha}\left[1+\lambda\left(V_{D S}-V_{D D}\right)\right], & \left(V_{D S} \geq V_{D S A T}: \text { saturation }\right),\end{cases}
$$

where $\beta_{s}=\frac{I_{D 0}}{\left(V_{D D}-V_{t h}\right)^{\alpha}}, \beta_{l}=\frac{\beta_{s}\left[1+\lambda\left(V_{D S A T}-V_{D D}\right)\right]}{V_{D S A T}}$. Here $\alpha$ is the velocity saturation index and is taken to be $\alpha=1$ for the technology nodes considered. $\lambda$ is the empirical channel length modulation factor. $I_{D 0}$ is the drain current at $V_{G S}=V_{D S}=V_{D D} . V_{D S A T}$ is the drain saturation voltage at $V_{G S}=V_{D D} . V_{D S A T}$ is also considered to be the saturation voltage for all $V_{G S}$ as in [21], because the range of $V_{D D}$ is very small for technologies under $45 \mathrm{~nm}$ and $V_{D S A T}$ does not vary much in this range.

The $I_{D S}$ vs $V_{D S}$ curves for different values of $V_{G S}$ based on the current equation (3.1) are plotted for $45 \mathrm{~nm}$ technology in Figure 3.1. The estimated saturation current matches the HSPICE simulated current within 5\% error. Next the method to estimate delay using the above current equations is described. 


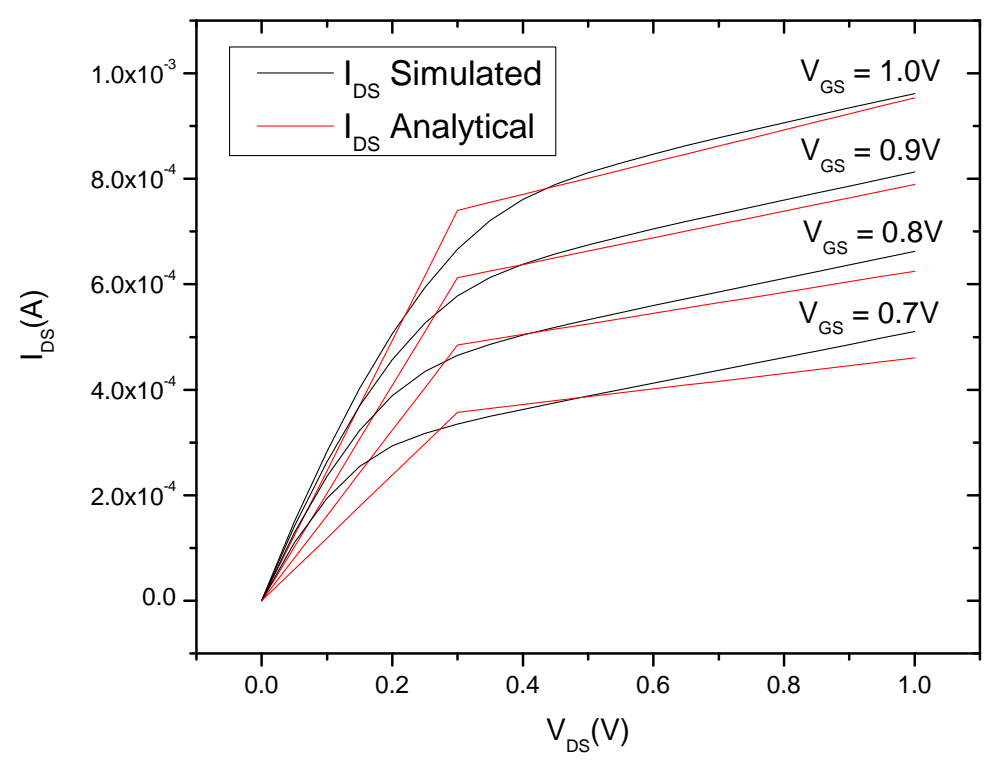

Figure 3.1: NMOS characteristics - Simulated and Analytical

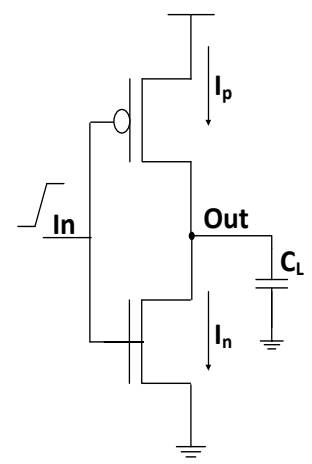

Figure 3.2: Schematic of CMOS Inverter circuit.

\subsubsection{Model derivation}

Input $V_{\text {in }}$ is considered to be a linear rising ramp input with transition time First, the delay equation is derived for high to low delay, $T_{p h l}$. The same equation is applicable to low to high delay. The input $V_{i n}$ is considered to be a linear rising ramp input with transition time $t_{r}$. So at time $\mathrm{t}, V_{i n}(t)=V_{D D} \times t / t_{r}$. As input ramps up, the region of operation of NMOS changes as shown in Figure 3.3. So, output voltage is derived based on the characteristics of the specific region. 


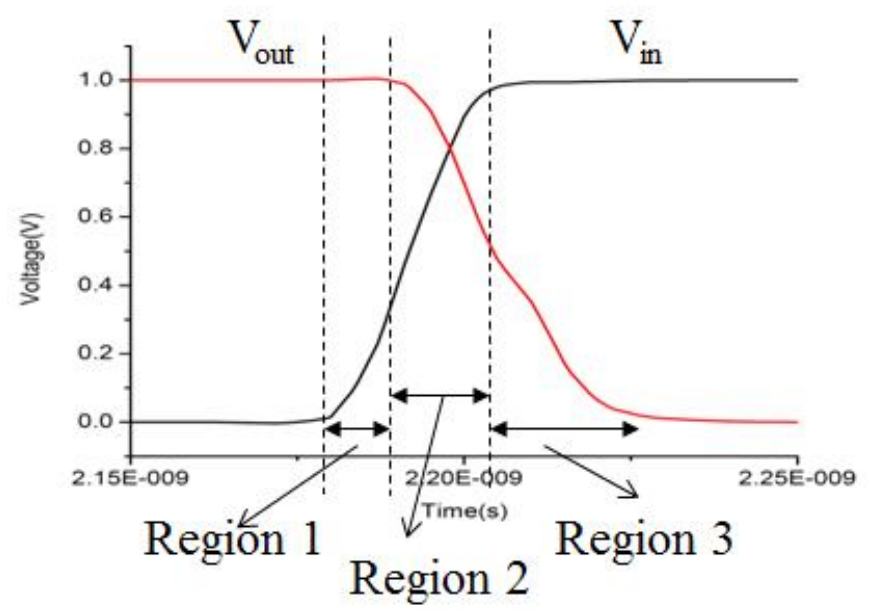

Figure 3.3: Regions of operation of NMOS transistor as input rises.

Region 1. $V_{\text {in }}<V_{t h}$ : Here NMOS is in cutoff region and no current flows through it. So output voltage is at $V_{D D}$.

Region 2. $V_{t h}<V_{i n} \leq V_{D D}$ : NMOS is in saturation and the output node starts discharging. Our derivation is different from [21] since we do not consider coupling capacitance. The contribution of coupling capacitance to delay is significant only if input has short transition times. But most of the gates derive their input from previous stage and do not have sharp edges. So it is unnecessary to consider coupling capacitance and make the derivation more complicated. The current to voltage relation in an inverter is

$$
\frac{d V_{\text {out }}}{d t} C_{L}=-I_{n}+I_{p}
$$

where $C_{L}$ is the load capacitance at output node, $I_{n}$ is current through the NMOS and $I_{p}$ is current through the PMOS, as shown in Figure 3.2. In this region, while $V_{i n}<V_{D D}-V_{t h p}$, where $V_{t h p}$ is the threshold voltage of the PMOS, PMOS is in the linear region. The time during which both NMOS and PMOS are on is when $V_{t h}<V_{i n}<V_{D D}-V_{t h p}$. For scaled technologies, this period is very small because $V_{D D}$ is small and $V_{D D}-V_{t h p}-V_{t h}$ approaches zero. This is different compared to previous technologies where $V_{D D}$ was large enough to keep both PMOS and NMOS on for sufficient time to affect propagation delay. So here PMOS current is ignored unlike [21].

With these new conditions, a new set of equations are derived. The above differential 
equation (3.2) is solved for $V_{\text {out }}$ by substituting saturation current equation from (3.1) to get

$$
V_{\text {out }}=\left(V_{D D}-\frac{1}{\lambda}\right)\left(e^{K_{y}\left(V_{\text {in }}-V_{t h}\right)^{\alpha+1}}\right)+K
$$

where $K_{y}=\frac{\beta_{s} t_{r} \lambda}{C_{L}(\alpha+1) V_{D D}}$. Constant $K$ is found from the boundary condition when $V_{i n}=V_{t h}$. The corresponding $V_{\text {out }}$ is $V_{D D}$ and $K=1 / \lambda$. So the final equation for $V_{\text {out }}$ is

$$
V_{\text {out }}=\left(V_{D D}-\frac{1}{\lambda}\right)\left(e^{K_{y}\left(V_{\text {in }}-V_{\text {th }}\right)^{\alpha+1}}\right)+\frac{1}{\lambda}
$$

Region 3. $t>t_{r}, V_{i n}=V_{D D}$ : In this region, NMOS is still in saturation and PMOS is in cutoff. The output node continues to discharge and reaches $V_{D D} / 2$. Saturation current equation from (3.1) with $V_{i n}=V_{D D}$ is applied to equation (3.2) to get

$$
V_{\text {out }}=\left(V_{D D}-\frac{1}{\lambda}\right)\left(e^{K_{z} t}\right)+K_{2}
$$

where $K_{z}=\frac{\beta_{s} \lambda\left(V_{D D}-V_{t h}\right)^{\alpha}}{C_{L}}$. Constant $K_{2}$ is found from the boundary condition when $t=t_{r}$. By equating $V_{\text {out }}$ from equation (3.5) to that from equation (3.4), we get

$$
K_{2}=\left(V_{D D}-\frac{1}{\lambda}\right)\left(\left(e^{K_{y}\left(V_{D D}-V_{t h}\right)^{\alpha+1}}\right)-e^{K_{z} t_{r}}\right)+\frac{1}{\lambda}
$$

With technology scaling, propagation delays have reduced to the order of transition times. So transition times can no longer be ignored in the delay equations. Propagation delay, $T_{p h l}$ is defined by the time between when $V_{i n}=V_{D D} / 2$ (that is $t_{r} / 2$ ) and when $V_{\text {out }}=V_{D D} / 2$. $V_{\text {out }}$ reaches $V_{D D} / 2$ in either Region 2 or Region 3, depending on the input transition time and output load capacitance. Thus the expression for $T_{p h l}$ depends on whether the input transition time is small or large, or whether the output load capacitance is small or large.

- For slow input or small load capacitance, $V_{\text {out }}$ reaches $V_{D D} / 2$ in Region 2. When $V_{\text {out }}$ is $V_{D D} / 2$, from equation (3.4), $V_{D D} / 2=\left(V_{D D}-\frac{1}{\lambda}\right)\left(e^{K_{y}\left(V_{D D} t / t_{r}-V_{t h}\right)^{\alpha+1}}\right)+\frac{1}{\lambda}$. For $\alpha=1$, solving for $\mathrm{t}$, we get $t=\frac{t_{r}}{V_{D D}}\left[\sqrt{K_{l o g}}+V_{t h}\right]$.

$T_{p h l}=t-t_{r} / 2$. So,

$$
T_{p h l}=\frac{t_{r}}{V_{D D}}\left[\sqrt{K_{l o g}}+V_{t h}\right]-\frac{t_{r}}{2}
$$

where $K_{l o g}=\frac{1}{K_{y}} \ln \left[\frac{0.5 V_{D D}-\frac{1}{\lambda}}{V_{D D}-\frac{1}{\lambda}}\right]$. 
- For fast input or large load capacitance, $V_{\text {out }}$ reaches $V_{D D} / 2$ in Region 3. $T_{p h l}$ is obtained in a similar way but now using equation (3.5) and is given by

$$
T_{p h l}=\frac{1}{K_{z}} \ln \left[\frac{0.5 V_{D D}-K_{2}}{V_{D D}-\frac{1}{\lambda}}\right]-\frac{t_{r}}{2}
$$

\subsubsection{Model Validation}

The model is validated for a wide range of widths, load capacitances and transition times with HSPICE simulations. First, width is varied from twice minimum length to 20 times minimum length and for this case, fanin and fanout are fixed at FO4. Next load capacitance is varied by sweeping fanout from FO4 to FO20 and keeping fanin to be FO4. Here width of inverter is fixed at 4 times minimum length. Then input transition time is varied by sweeping fanin of the gate with fanout fixed at 10 . Here too width of inverter is fixed to be 4 times minimum length.

Figures 3.4 and 3.5 plot high to low (HL) delay values predicted by the model and HSPICE simulation results for $45 \mathrm{~nm}$ and $32 \mathrm{~nm}$ technologies, respectively. As seen from Figures 3.4 and 3.5, delay is almost constant with varying width, as expected. Delay is proportional to load capacitance and it is also proportional to transition time for small transition times but saturates for large transition times. Figure 3.4 also shows that model is continuous between Region 2 and Region 3. The analytical model for nominal delay matches the simulated values with average error of $1.08 \%$ when varying width, $2.95 \%$ error when varying load capacitance and $1.83 \%$ when varying transition time for $45 \mathrm{~nm}$ technology. For $32 \mathrm{~nm}$ technology, average errors are $0.71 \%, 4.58 \%$ and $3.15 \%$ with varying width, load capacitance and input transition times, respectively.

Next for low to high (LH) delay, the equation for $T_{p l h}$, is similar to that of $T_{p h l}$; the NMOS parameters such as $V_{t h}, I_{D 0}$, width and $V_{D S A T}$ are replaced by the corresponding PMOS parameter. LH delay plots are generated for varying widths, load capacitance and transition times. They are shown in Figures 3.6 and 3.7 for $45 \mathrm{~nm}$ and 32nm technology nodes, respectively. As seen from Figures 3.6 and 3.7, the model matches the predicted value closely. The average error in $45 \mathrm{~nm}$ technology when sweeping width is $-1.23 \%$, when sweeping $C_{L}$ is $-5.23 \%$ and when sweeping $t_{r}$ is $2.9 \%$. The average error in $32 \mathrm{~nm}$ technology when sweeping width is 


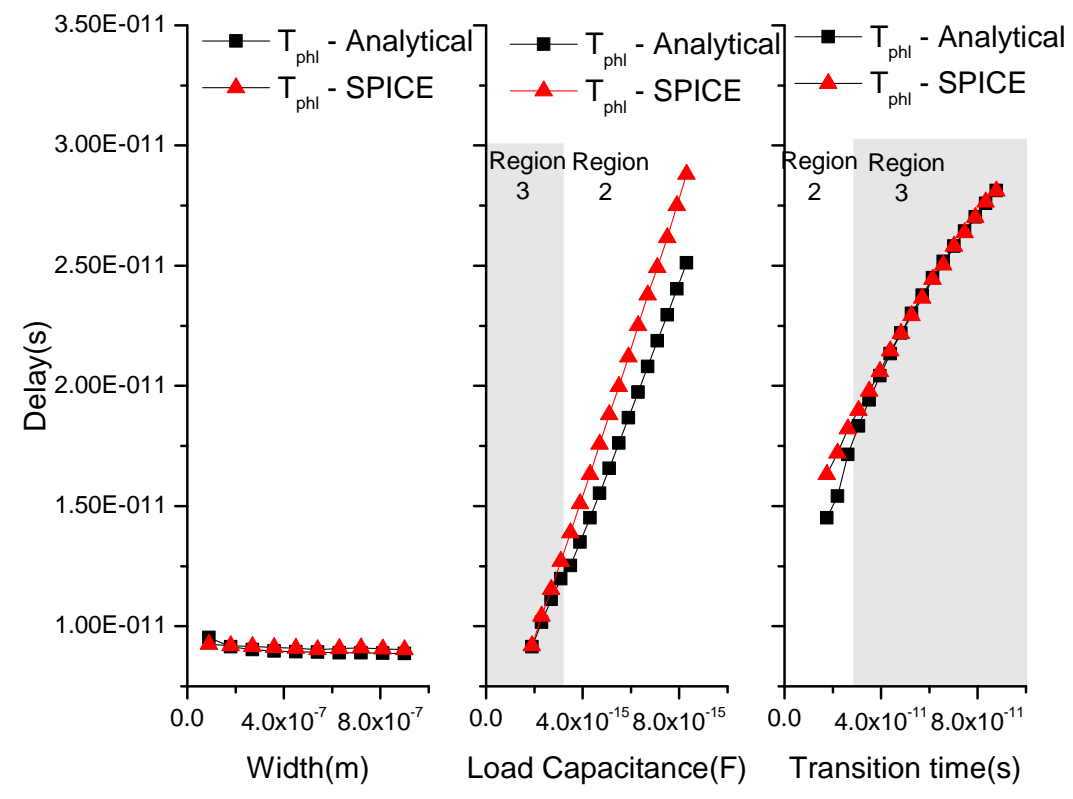

Figure 3.4: Inverter HL delay with varying width, capacitance, transition time at $45 \mathrm{~nm}$ technology node.

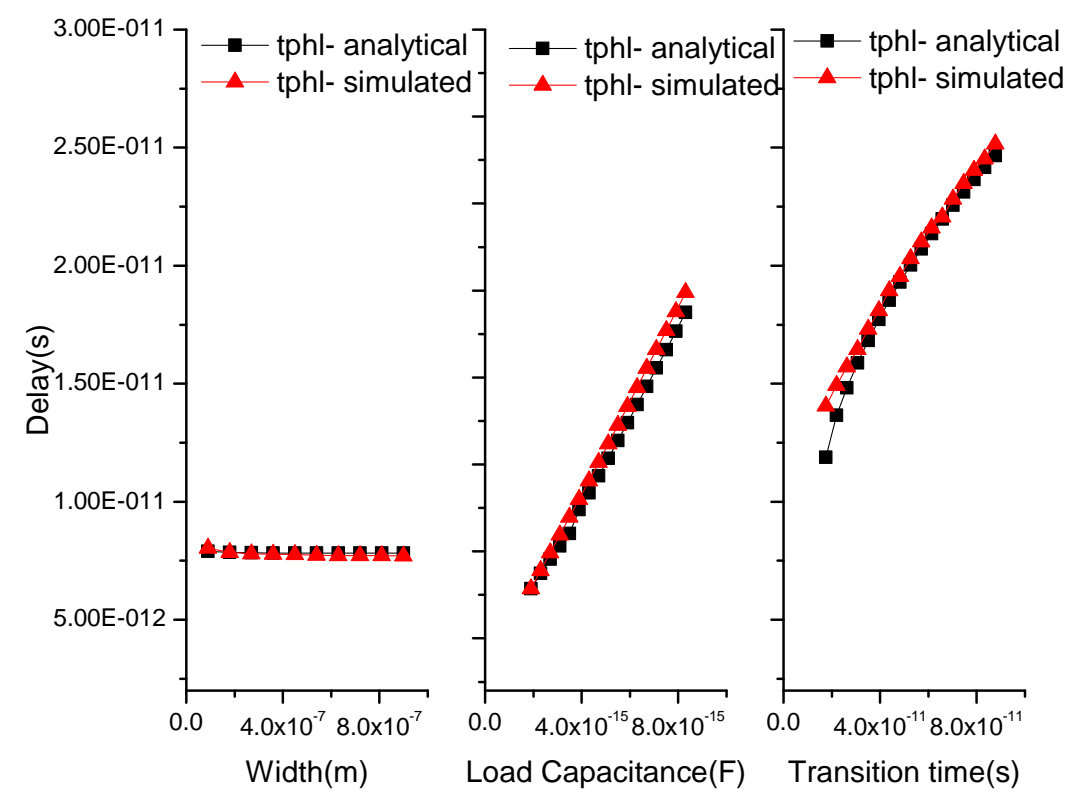

Figure 3.5: Inverter HL delay with varying width, capacitance, transition time at 32nm technology node. 
$-4.0 \%$, when sweeping $C_{L}$ is $-4.3 \%$ and when sweeping $t_{r}$ is $1.4 \%$. Thus the proposed model is accurate for predicting nominal delay of inverter.

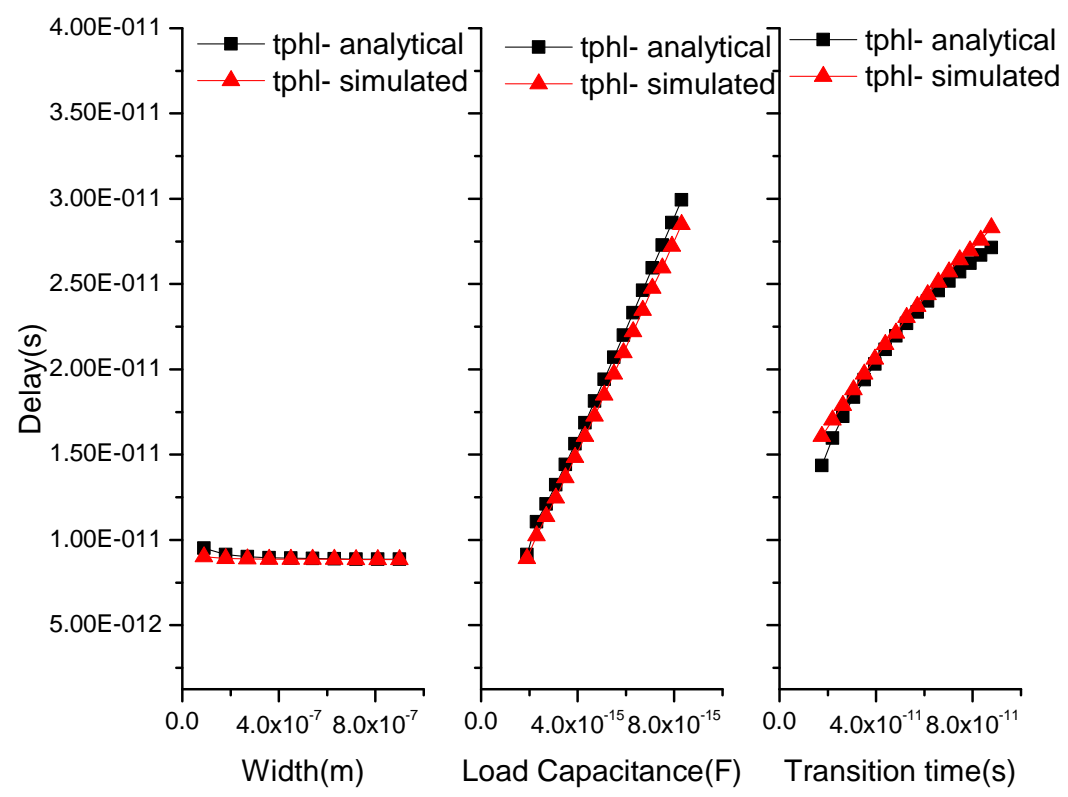

Figure 3.6: Inverter LH delay with varying width, capacitance, transition time at $45 \mathrm{~nm}$ technology node.

\subsection{Nominal Delay Model for NAND and NOR gates}

The delay model derived for an inverter is extended to handle stacked transistors in NAND and NOR gates. First the output voltage behavior is modeled according to region of operation of NMOS and PMOS transistors and then the $T_{p h l}$ delay is found by the time between $t_{r} / 2$ and the time when $V_{\text {out }}$ reaches $V_{D D} / 2$. The $T_{p h l}$ delay equations for NAND2 gate are derived, and the same equations can be applied to NOR2 gate also. Delay equations for NAND3 are also given at the end of this section with supporting simulation results and plots.

\subsubsection{NAND2 Delay Model}

In stacked transistors, output voltage discharge characteristics depends on state of the transistors placed between the transistor with switching input and the output. Transistors placed between switching input and supply nodes do not affect output and hence delay. For instance, in Figure 3.8, when input is given to A1, output depends only on transistor M1. But when input is given to A2, output depends on both M1 and M2 transistors. For the NAND2 gate the two cases 


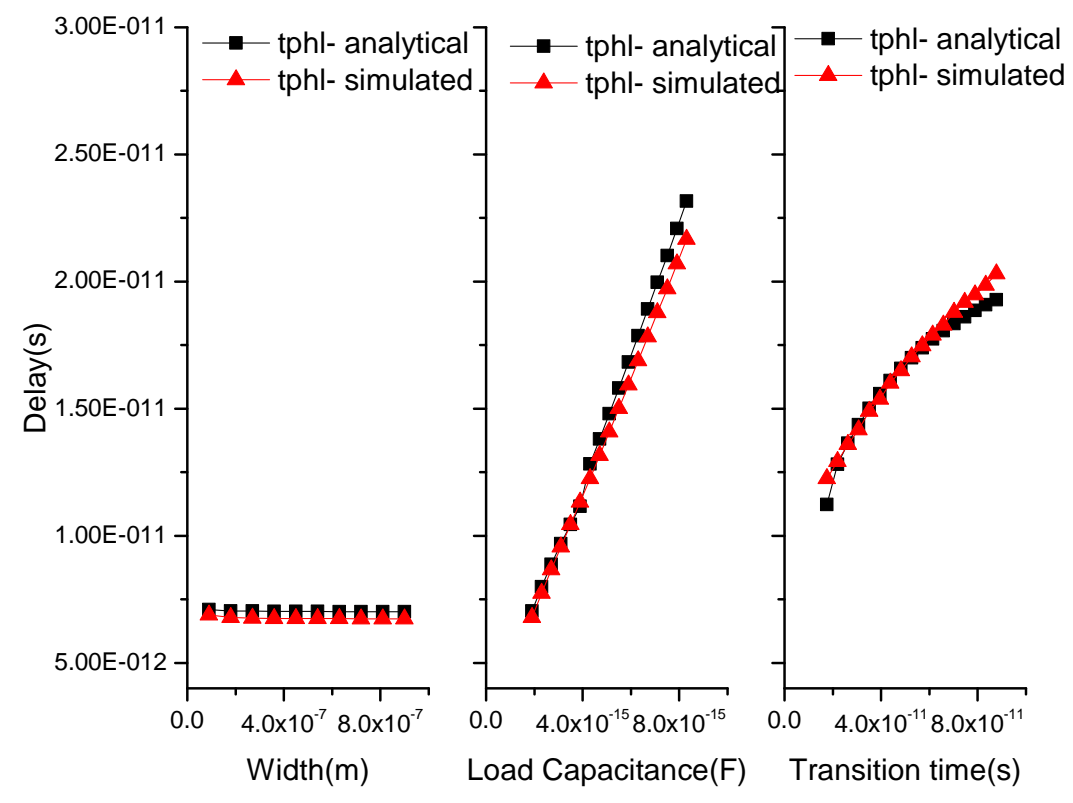

Figure 3.7: Inverter LH delay with varying width, capacitance, transition time at 32nm technology node.

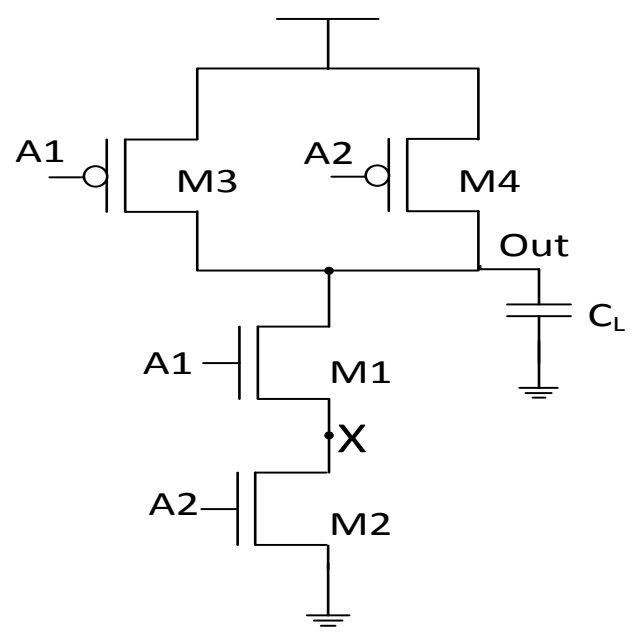

Figure 3.8: NAND2 gate schematics.

are considered separately:

Case 1. Input given to bottom transistor: We assume that input voltage rises from 0 to $V_{D D}$ in transition time $t_{r}$. Initially when input voltage is at $0 \mathrm{~V}$, output voltage is at $V_{D D}$. The voltage at node $X$ in Figure 3.8 is at $V_{D D}-V_{t h, M 1}$, where $V_{t h, M 1}$ is the threshold voltage of M1. According 


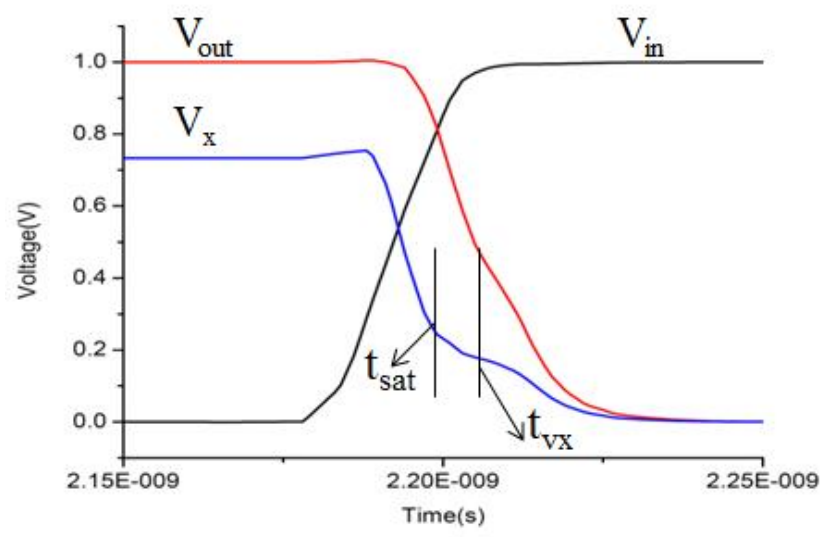

Figure 3.9: NAND2 gate discharge behavior when input is given to bottom transistor.

to Elmore's law, delay is proportional to

$$
R_{2}\left(C_{L}+C_{X}\right)+R_{1} C_{L}
$$

where $C_{L}$ is the load capacitance and $C_{X}$ is the capacitance at node $X$. The first term, in equation (3.9), $t_{v x}=R_{2}\left(C_{L}+C_{X}\right)$, is the time to discharge $C_{L}$ and $C_{X}$ through M2. The second term, $t_{\text {vout }}=R_{1} C_{L}$, is the time to discharge load capacitance through M1. So, $T_{p h l}$ of NAND2 gate when input is give to bottom transistor is

$$
T_{p h l}=t_{v x}+t_{v o u t}-\frac{t_{r}}{2}
$$

- Derivation of $t_{v x}$ : As input to M2 increases, M2 shifts from cut-off region to saturation. It moves to linear region when $V_{x}$ discharges below $V_{D S A T}$. Let $V_{x f}$ be the final voltage at $X$ when $V_{\text {out }}$ reaches $V_{D D} / 2$. So total time taken to discharge $C_{L}+C_{X}$ through M2 can be split into two:

1. Time taken for $V_{X}$ to discharge from $V_{D D}-V_{t h, M 1}$ to $V_{D S A T}, t_{s a t}$. Here M2 is in saturation.

2. Time taken to discharge from $V_{D S A T}$ to $V_{x f}$. Here $\mathrm{M} 2$ is in linear region.

These times are shown in Figure 3.9. From equation (3.7),

$$
t_{s a t}=\frac{t_{r}}{V_{D D}}\left[\sqrt{K_{l o g}}+V_{t h}\right]
$$


where $K_{\log }=\frac{1}{K_{y}} \ln \left[\frac{V_{D S A T}-\frac{1}{\lambda}}{V_{D D}-\frac{1}{\lambda}}\right]$. Depending on input transition time, $t_{s a t}$ and $t_{v x}$ can be less than $t_{r}$ or more than $t_{r}$.

$\mathbf{t}_{\mathbf{v x}}<\mathbf{t}_{\mathbf{r}}$ : In this case input is still rising when $V_{x}$ reached $V_{x f}$ and M2 is in linear region. Using equation (3.2), $V_{\text {out }}$ is solved with $I_{n}$ represented by linear current equation.

$$
V_{\text {out }}=e^{\left[-K_{x}\left(\frac{V_{D D^{t}}}{t_{r}}-V_{t h}\right)^{\alpha+1}-C\right]},
$$

where $K_{x}=\frac{\beta_{s} t_{r}\left[1+\lambda\left(V_{D S A T}-V_{D D}\right)\right]}{V_{D D} C_{L} V_{D S A T}(\alpha+1)}$. The constant $\mathrm{C}$ is found using the boundary condition when $V_{\text {out }}$ is equal to $V_{D S A T}$ at $t=t_{\text {sat }}$.

Time when $V_{\text {out }}$ reaches $V_{x f}$ is

$$
t_{v x}=\frac{t_{r}}{V_{D D}}\left[\sqrt{\frac{\ln \left(V_{x f}\right)+C}{-K_{x}}}+V_{t h}\right],
$$

where $C=-K_{x}\left(\frac{V_{D D} t_{s a t}}{t_{r}}-V_{t h}\right)^{\alpha+1}-\ln \left(V_{D S A T}\right)$.

$\mathbf{t}_{\mathbf{s a t}} \leq \mathbf{t}_{\mathbf{r}}, \mathbf{t}_{\mathbf{v x}} \geq \mathbf{t r}:$ During the time from $t_{s a t}$ to $t_{r}$, M2 is in linear region with rising input and voltage at $V_{x}$ is given by equation (3.12). Let the voltage at $V_{x}$ reach $V_{x, t r}$ when $t=t_{r}$. The time taken to discharge $V_{x}$ from $V_{x, t r}$ to $V_{x f}$ is $\ln \left(\frac{V_{x, t r}}{V_{x f}}\right) R_{2} C_{x}$, where $R_{2}=\frac{V_{D S}}{I_{D 0}}=\frac{V_{D S A T}}{\left[\beta_{s}\left(1+\lambda\left(V_{D S A T}-V_{D D}\right)\right)\right]\left(V_{D D}-V_{t h}\right)}$. So total $t_{v x}$ is given by equation (3.14).

$$
t_{v x}=t_{r}+\ln \left(\frac{V_{x f}}{V_{x, t r}}\right) R_{2} C_{x}
$$

$\mathbf{t}_{\text {sat }}>\mathbf{t}_{\mathbf{r}}:$ Here input voltage has already reached $V_{D D}$. So time taken to discharge from $V_{D S A T}$ to $V_{x f}$ is given by $\ln \left(\frac{V_{D S A T}}{V_{x f}}\right) R_{2} C_{x}$. The total $t_{v x}$ is given by equation (3.15).

$$
t_{v x}=t_{s a t}+\ln \left(\frac{V_{D S A T}}{V_{x f}}\right) R_{2} C_{x}
$$

where $R_{2}=\frac{V_{D S}}{I_{D 0}}=\frac{V_{D S A T}}{\left[\beta_{s}\left(1+\lambda\left(V_{D S A T}-V_{D D}\right)\right)\right]\left(V_{D D}-V_{t h}\right)}$ and $t_{s a t}$ is given by (3.11).

- Derivation of $t_{\text {vout }}$ : During the discharge of output as well as $X$ nodes, M1 is always in linear region. So it acts as a simple resistor whose resistance can be derived from linear current equation in (3.1).

$$
R_{1}=\frac{V_{D S}}{I_{D 0}}=\frac{V_{D S A T}}{\left[\beta_{s}\left(1+\lambda\left(V_{D S A T}-V_{D D}\right)\right)\right]\left(V_{D D}-V_{t h}^{\prime}\right)}
$$

Here $V_{t h}^{\prime}$ is threshold voltage of M1 or M2 depending on if the input has fast or slow transition time. When input has fast transition edge $\left(t_{r}<t_{\text {sat }}\right)$, current through M2 is 
large, current through M1 is limited by M1 itself and $V_{t h}^{\prime}=V_{t h, M 1}$. If input has slow transition edge, current through M2 is small and current through M1 is limited by M2. So $V_{t h}^{\prime}=V_{t h}$. The time to discharge $C_{L}$ from $V_{D D}$ to $V_{D D} / 2$ is given by

$$
t_{\text {vout }}=0.69 R_{1} C_{L} \text {. }
$$

Case 2. Input given to top transistor: When input is given to top transistor, $V_{X}$ is already discharged. So only $V_{\text {out }}$ has to discharge from $V_{D D}$ to $V_{D D} / 2$ through the stack. This is equivalent to an inverter where M1 and M2 are together and represented by a single transistor of almost half the width. The delay is given by the equations (3.7) or (3.8) depending on whether the input is fast or slow.

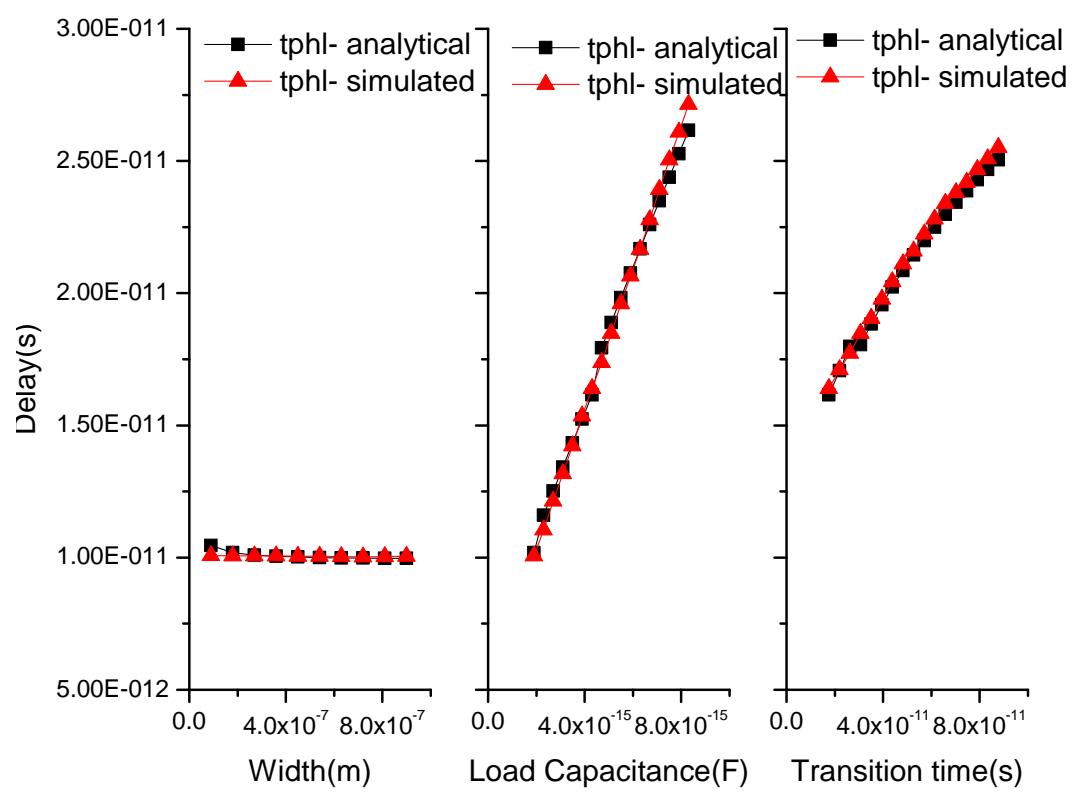

Figure 3.10: NAND2 gate HL delay with varying width, capacitance, transition time when input is given to M2 at $45 \mathrm{~nm}$ technology node.

\subsubsection{Model Validation}

The plots in Figures 3.10 and 3.11 show the results using the proposed model and HSPICE simulations for NAND2 gate when input is given to M2(bottom) and M1(top) respectively. Delay values are plotted for varying widths, load capacitances and transition times. Similar to inverter plots, fanin and fanout are kept constant at FO4 while sweeping width from 2 times 


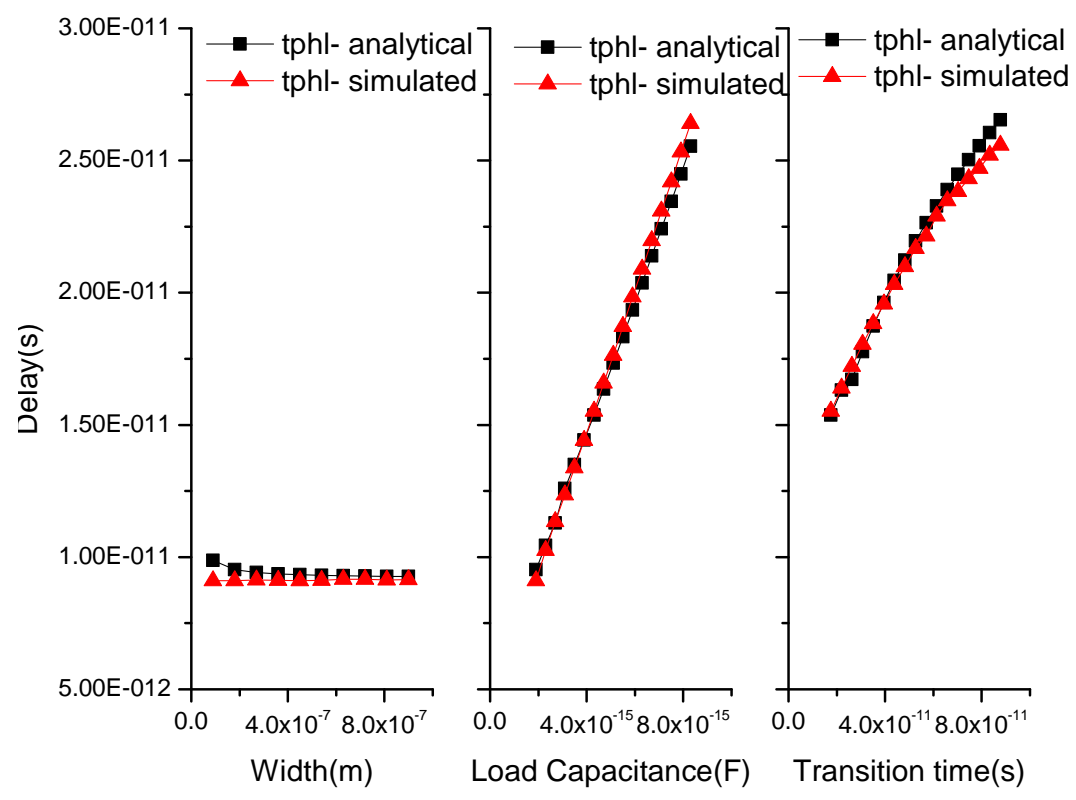

Figure 3.11: NAND2 gate HL delay with varying width, capacitance, transition time when input is given to $\mathrm{M} 1$ at $45 \mathrm{~nm}$ technology node.

minimum width to 20 times minimum width. For varying load capacitance, fanout is swept from $\mathrm{FO} 4$ to FO20, while fanin is kept at FO4 and width set at 4 times minimum length. For varying input transition time, fanin is swept from FO4 to FO20, while fanout is fixed at FO10 and width is fixed at 4 times minimum width.

Similar to INV delay characteristics, delay in NAND2 gate is also almost invariant to width, varies linearly with $C_{L}$ and varies linearly with $t_{r}$ for low transition times and saturates for higher values. When input is given to M2 the average error when varying width is $-0.16 \%$, when varying $C_{L}$ is $-0.27 \%$ and when varying $t_{r}$ is $1.17 \%$. When input is given to M1 the average error when varying width is $-2.92 \%$, when varying $C_{L}$ is $1.02 \%$ and when varying $t_{r}$ is $-1.12 \%$.

Similar plots are generated for NOR2 gate but for low to high delays. The average error when input is given to M2(top) when varying width is $-5.15 \%$, when varying $C_{L}$ is $-0.66 \%$ and when varying $t_{r}$ is $-0.31 \%$. The average error when input is given to M1(bottom) when varying width is $-2.45 \%$, when varying $C_{L}$ is $-1.29 \%$ and when varying $t_{r}$ is $1.36 \%$. 


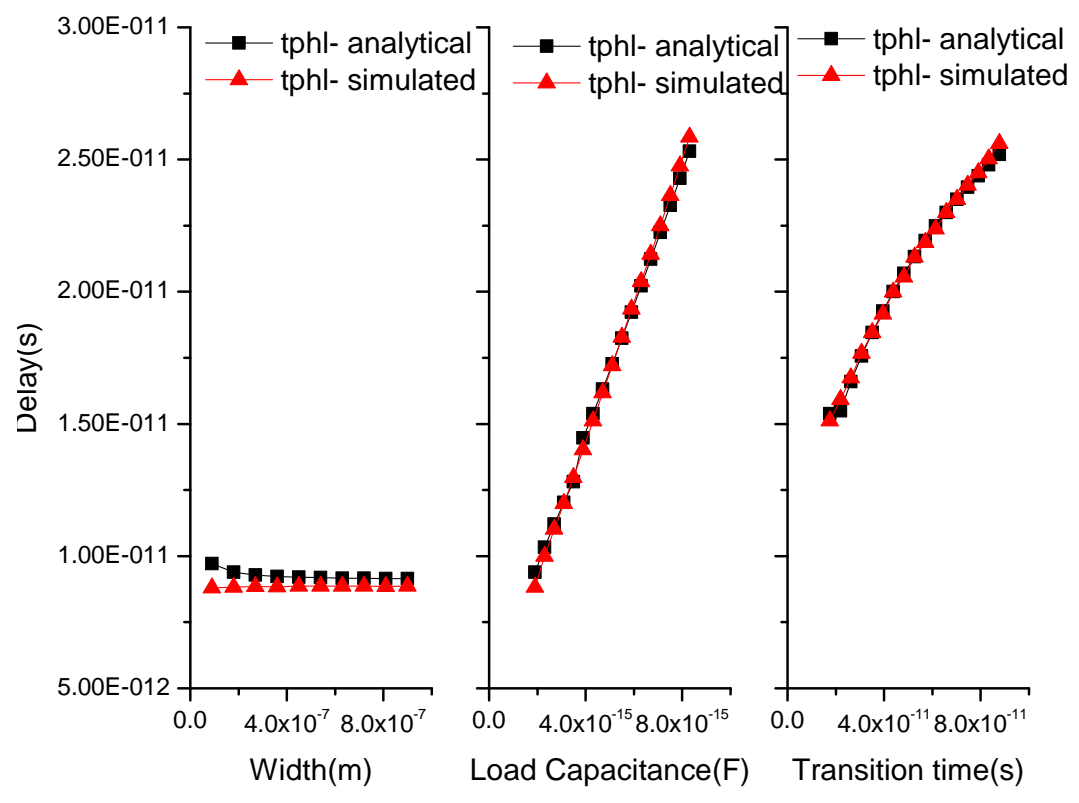

Figure 3.12: NOR2 gate LH delay with varying width, capacitance, transition time when input is given to bottom PMOS at $45 \mathrm{~nm}$ technology node.

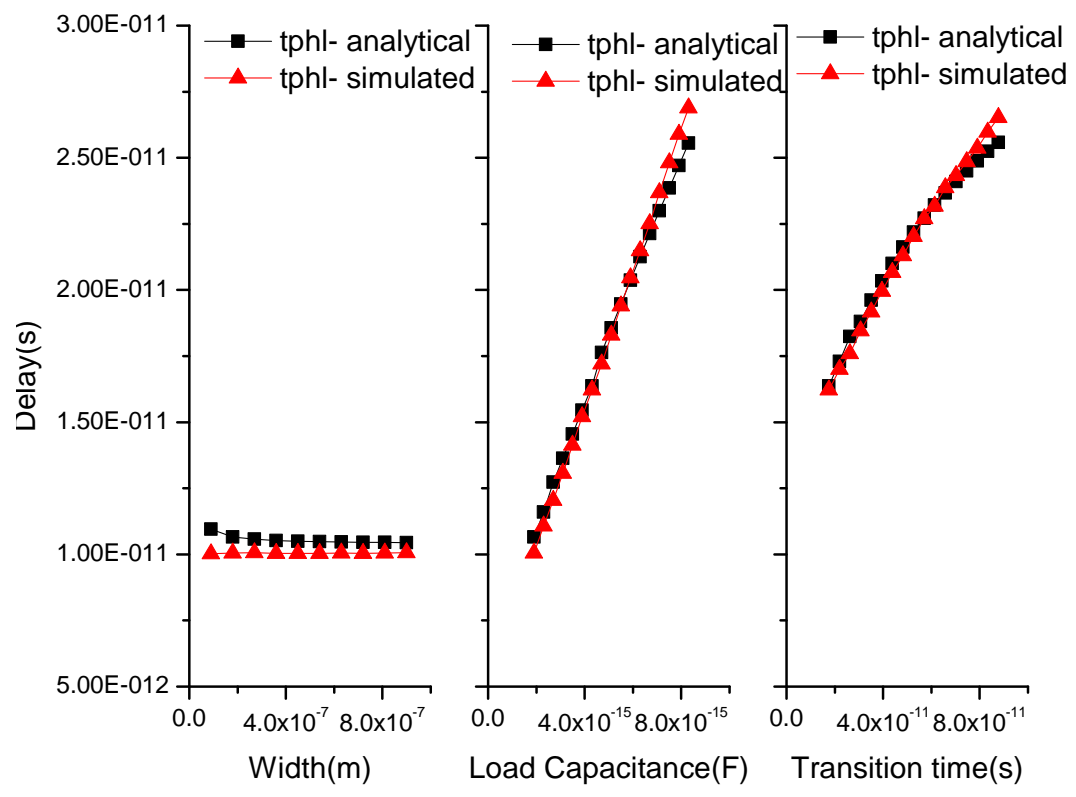

Figure 3.13: NOR2 gate LH delay with varying width, capacitance, transition time when input is given to top PMOS at $45 \mathrm{~nm}$ technology node. 
The low to high delays for NAND gates and high to low delays for NOR gates follow the exact same equations for inverter because transistors are not stacked here and are equivalent to inverters.

\subsubsection{NAND3 Delay Model}

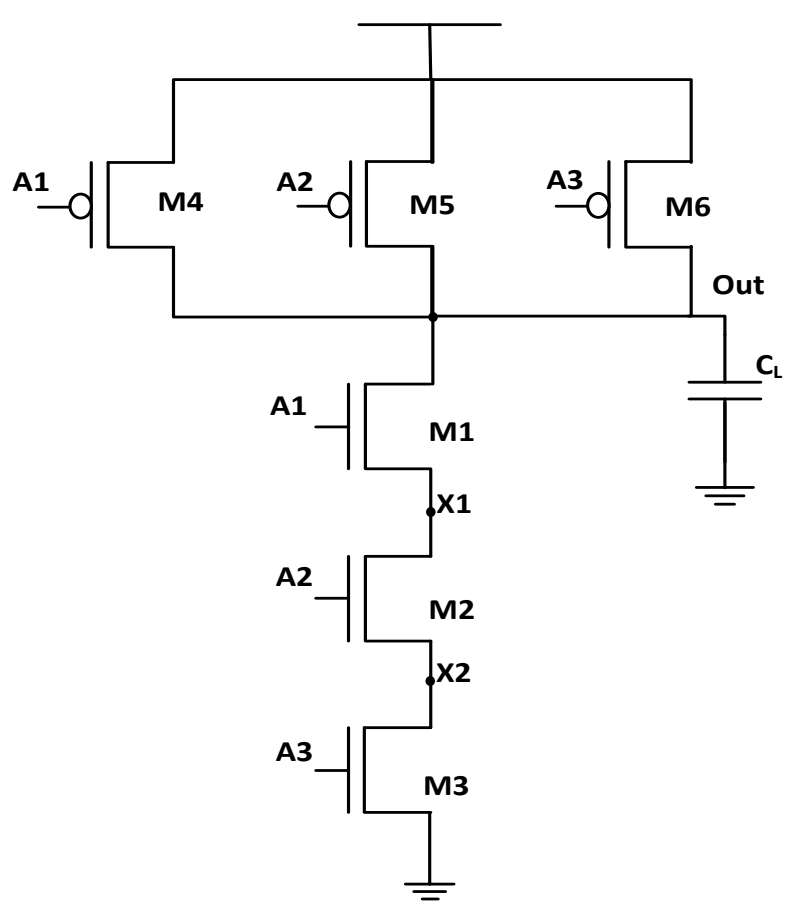

Figure 3.14: NAND3 gate schematics.

Delay equations for NAND3 are derived using a similar procedure. Figure 3.14 shows a NAND3 gate where M1 is the top transistor, M2 is the middle transistor and M3 is the bottom transistor.

Case 1. Input given to M1: This is the simplest case where nodes $\mathrm{X} 1$ and $\mathrm{X} 2$ are already discharged and the voltage at the output node has to discharge through the three transistors. M1, M2 and M3 are reduced to an equivalent transistor of almost one-third the width of NAND3 gate NMOS. The delay equation is similar to inverter delay given by equation (3.7) or (3.8) depending on input slew rate.

Case 2. Input given to M2: In this case, $\mathrm{X} 2$ is already discharged but $\mathrm{X} 1$ and output node have 
to be discharged. Delay depends on M2 and M1. It is given by sum of $t_{v x 1}$ and $t_{v o u t}$.

$$
T_{p h l}=t_{v x 1}+t_{v o u t}-\frac{t_{r}}{2}
$$

$t_{v x 1}$ is given by one of the equations (3.13), (3.14) and (3.15) depending on the input slew rate. $t_{\text {vout }}$ is given by

$$
t_{\text {vout }}=0.69 R_{1} C_{L}
$$

where $R_{1}=\frac{V_{D S}}{I_{D 0}}=\frac{V_{D S A T}}{\left[\beta_{s}\left(1+\lambda\left(V_{D S A T}-V_{D D}\right)\right)\right]\left(V_{G S}-V_{t h}\right)}$

Case 3. Input given to M3: In this case both $\mathrm{X} 1$ and $\mathrm{X} 2$ are charged and delay depends on all the three transistors M1, M2 and M3.

$$
T_{p h l}=t_{v x 1}+t_{v x 2}+t_{v o u t}-\frac{t_{r}}{2}
$$

$t_{v x 2}$ is given by one of the equations (3.13), (3.14) and (3.15) depending on the input slew rate. $t_{v x 1}$ is similar to $t_{\text {vout }}$ because $\mathrm{M} 2$ is also in linear region all through the discharge of $V_{\text {out }} . R C$ constant is multiplied by 0.4 because there is only around $30 \%$ discharge. Thus $t_{v \times 2}$ is given by

$$
t_{v x 2}=0.4 R_{2}\left(C_{L}+C_{x 1}\right)
$$

where $R_{2}=\frac{V_{D S}}{I_{D 0}}=\frac{V_{D S A T}}{\left[\beta_{s}\left(1+\lambda\left(V_{D S A T}-V_{D D}\right)\right)\right]\left(V_{G S 2}-V_{t h}\right)}$

Finally $t_{\text {vout }}$ is given by

$$
t_{\text {vout }}=0.69 R_{1} C_{L}
$$

where $R_{1}=\frac{V_{D S}}{I_{D 0}}=\frac{V_{D S A T}}{\left[\beta_{s}\left(1+\lambda\left(V_{D S A T}-V_{D D}\right)\right)\right]\left(V_{G S 1}-V_{t h}\right)}$

\subsubsection{NAND3 Validation}

The plots for NAND3 gate when input is given to top, middle and bottom transistors are given in Figures 3.15, 3.16 and 3.17, respectively. The average error when input is given to M1(top) when varying width is $-3.16 \%$, when varying $C_{L}$ is $1.01 \%$ and when varying $t_{r}$ is $0.44 \%$. The average error when input is given to M2(middle) when varying width is $-0.10 \%$, when varying $C_{L}$ is $2.69 \%$ and when varying $t_{r}$ is $0.58 \%$. The average error when input is given to M3(bottom) when varying width is $1.71 \%$, when varying $C_{L}$ is $1.91 \%$ and when varying $t_{r}$ is $1.01 \%$. 


\subsubsection{Summary:}

In this chapter we derived nominal delay models for inverter and stacked transistors such as NAND2, NOR2 and NAND3. Delay predicted is in good agreement with simulated results. Hence this approach can be extended to any complex circuit considering input transition time, load capacitance and stacking effect.

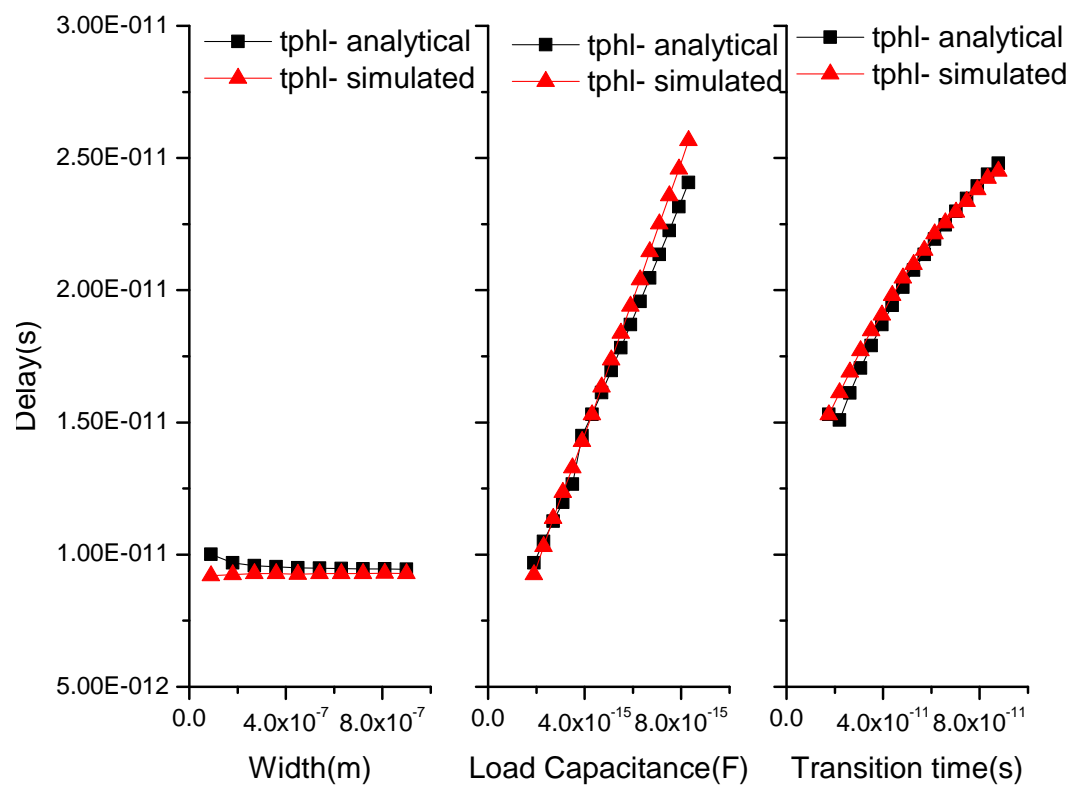

Figure 3.15: NAND3 gate HL delay with varying width, capacitance, transition time when input is given to M1 at $45 \mathrm{~nm}$ technology node. 


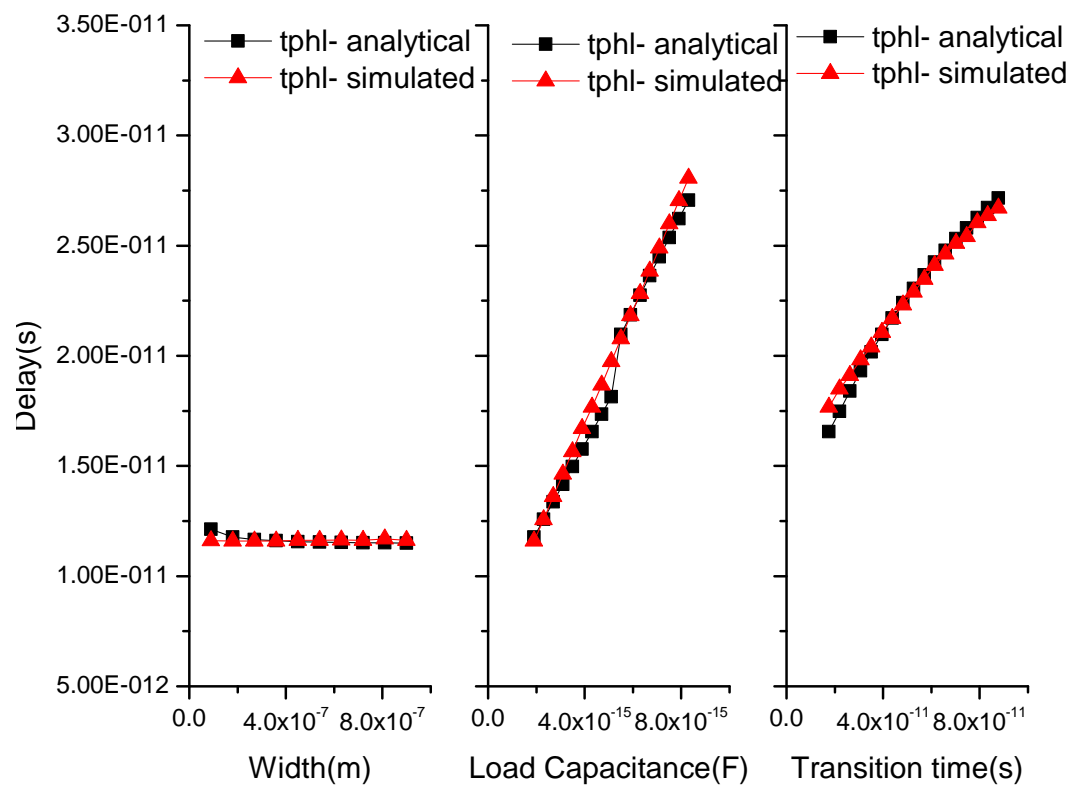

Figure 3.16: NAND3 gate HL delay with varying width, capacitance, transition time when input is given to $\mathrm{M} 2$ at $45 \mathrm{~nm}$ technology node.

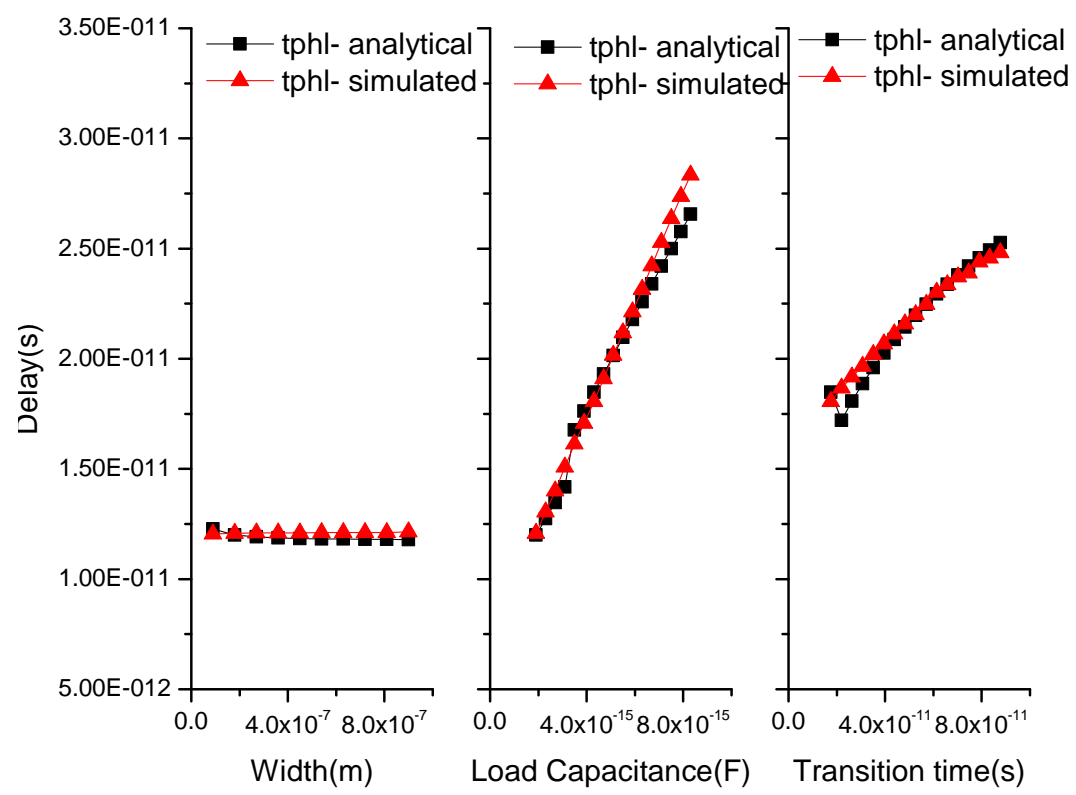

Figure 3.17: NAND3 gate HL delay with varying width, capacitance, transition time when input is given to M3 at $45 \mathrm{~nm}$ technology node. 
The parameters required in the model are given in Table 3.1. The parameters $\alpha, \lambda$, $V_{t h}, I_{D 0}$ and $V_{D S A T}$ are extracted from device characteristics. The parameters load capacitance and final voltage, $V_{x f}$, that node $\mathrm{X}$ reaches are parameters from the circuit level. All other parameters like $K_{y}, K_{z}, K_{2}, K_{l o g} C$ and $K_{x}$ are derived from the parameters in Table 3.1.

\begin{tabular}{|c|c|}
\hline Parameter & $\begin{array}{c}\text { Extraction } \\
\text { Information }\end{array}$ \\
\hline$\alpha$ & 1 for technologies considered \\
\hline$\lambda$ & Device characteristics \\
\hline$V_{t h}$ & Device characteristics \\
\hline$I_{D 0}$ & Device characteristics \\
\hline$V_{D S A T}$ & Device characteristics \\
\hline$C_{L}$ & Circuit characteristics \\
\hline$V_{x f}$ & Circuit characteristics \\
\hline
\end{tabular}

Table 3.1: Parameters used in the model and their extraction information. 


\section{Chapter 4}

\section{ANALYTICAL MODEL FOR DELAY VARIABILITY}

This chapter analyzes variation in delay due to variations in threshold voltage. The delay equations derived in Chapter 3 are used to predict delay variability. Delay variability of inverter is derived in Section 4.1. In Section 4.2 variability model is extended to NAND2 and NOR2 gates and later to NAND3 gates in Section 4.3. This model is validated with PTM models [4] at $45 \mathrm{~nm}$ technology and $32 \mathrm{~nm}$ technology nodes.

\subsection{Delay Variability in Inverter}

Threshold voltage variation in transistors is assumed to follow Gaussian distribution. So delay variation should also follow Gaussian distribution with standard deviation, $\sigma_{T_{p}}$ [28].

$$
\sigma_{T_{p}}=\frac{\partial\left(T_{p h l}\right)}{\partial V_{t h}} \sigma_{V_{t h}}
$$

Equation (4.1) can be applied on the delay equation of any gate to get delay variability due to $V_{t h}$ variation. For an inverter with slow rising input or small load capacitance, inverter delay follows equation (3.7) and variability in such case is given by

$$
\sigma_{T_{p}}=\frac{t_{r}}{V_{D D}} \sigma_{V_{t h}}
$$

For an inverter with fast rising inputs or large load capacitance, inverter delay follows equation (3.8) and variability in such case is given by

$$
\sigma_{T_{p}}=[S 1+S 2(S 3-S 4)] \sigma_{V_{t h}}
$$

where $S 1=\frac{1}{K_{z}\left(V_{D D}-V_{t h}\right)} \ln \left[\frac{0.5 V_{D D}-K_{2}}{V_{D D}-\frac{1}{\lambda}}\right], S 2=\frac{1}{K_{z}}\left[\frac{V_{D D}-\frac{1}{\lambda}}{0.5 V_{D D}-K_{2}}\right], S 3=\left(e^{K_{y}\left(V_{i n}-V_{t h}\right)^{\alpha+1}}\right)(\alpha+1) K_{y}\left(V_{D D}-\right.$ $\left.V_{t h}\right)^{\alpha}$ and $S 4=\frac{e^{K_{z} t_{r}} \lambda \beta_{s} t_{r}}{C_{L}}$. Here $\alpha$ is velocity saturation index and is taken to be $\alpha=1$ for technologies considered, $\lambda$ is the empirical channel length modulation factor, $\beta_{s}=\frac{I_{D 0}}{\left(V_{D D}-V_{t h}\right)^{\alpha}}$, $K_{y}=\frac{\beta_{s} t_{r} \lambda}{C_{L}(\alpha+1) V_{D D}}, K_{z}=\frac{\beta_{s} \lambda\left(V_{D D}-V_{t h}\right)^{\alpha}}{C_{L}}, K_{2}=\left(V_{D D}-\frac{1}{\lambda}\right)\left(\left(e^{K_{y}\left(V_{D D}-V_{t h}\right)^{\alpha+1}}\right)-e^{K_{z} t_{r}}\right)+\frac{1}{\lambda}$.

Delay variability obtained analytically is compared with HSPICE Monte Carlo simulations. Figures 4.1 and 4.2 show the variation in high to low(HL) delay with varying widths, load capacitances and input transition times for $45 \mathrm{~nm}$ and $32 \mathrm{~nm}$ technology nodes, respectively. The width of inverter is varied from two times minimum length to 20 times minimum length, 
keeping fanin and fanout constant at FO4. To study the effect of load capacitance, fanin and width are kept constant while varying load capacitance. To study the effect of input slew rate, fanout and width are constant while varying input transition times. Here the baseline voltage variation is $50 \mathrm{mV}$ corresponding to twice minimum width and the threshold voltage varies with width as $\sigma_{V_{t h}} \propto \frac{1}{\sqrt{W}}$, where $W$ is width of the transistor.

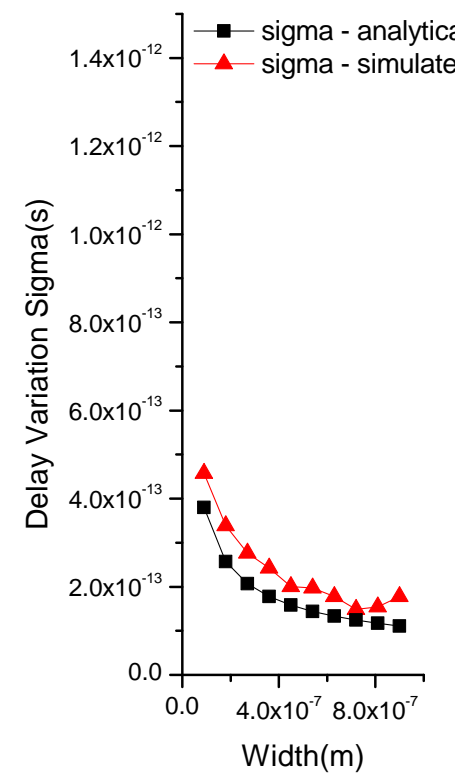

(a)

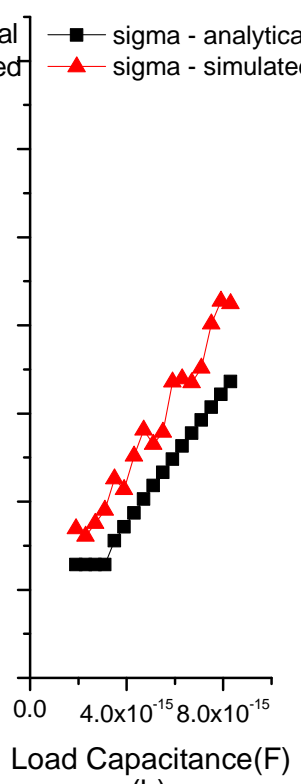

(b)

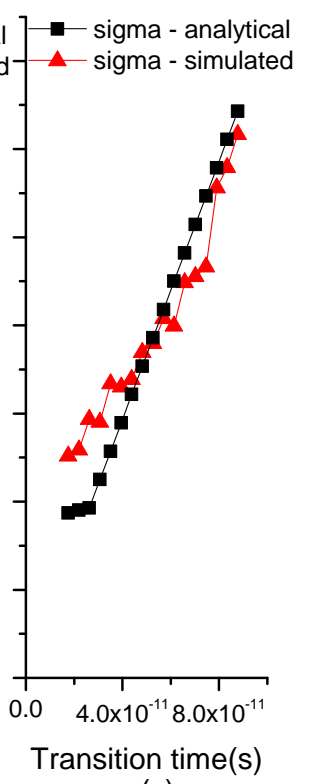

(c)

Figure 4.1: Inverter HL delay variation with varying (a) width, (b) capacitance, (c) transition time at $45 \mathrm{~nm}$ technology node.

Our observations are as follows.

- Since varying width does not have any effect on nominal delay (see Figure 3.4 ), $\sigma_{T_{p}}$ varies in proportion to $\sigma_{V_{t h}}$, as shown in Figures 4.1a and 4.2a.

- Increasing load capacitance makes equation (4.3) applicable. According to this equation, $S 1$ and $S 2$ are proportional to $\frac{1}{K_{z}}$, where $K_{z}$ is proportional to $\frac{1}{C_{L}}$. $S 3$ and $S 4$ vary almost similarly canceling out each others effect. So $\sigma_{T_{p}}$ varies linearly with $C_{L}$, as shown in Figures $4.1 \mathrm{~b}$ and $4.2 \mathrm{~b}$.

- Increasing input transition time is modeled by equation (4.2), according to which $\sigma_{T_{p}}$ is proportional to $t_{r}$. The trend is also very clearly seen in Figures 4.1c and 4.2c. 


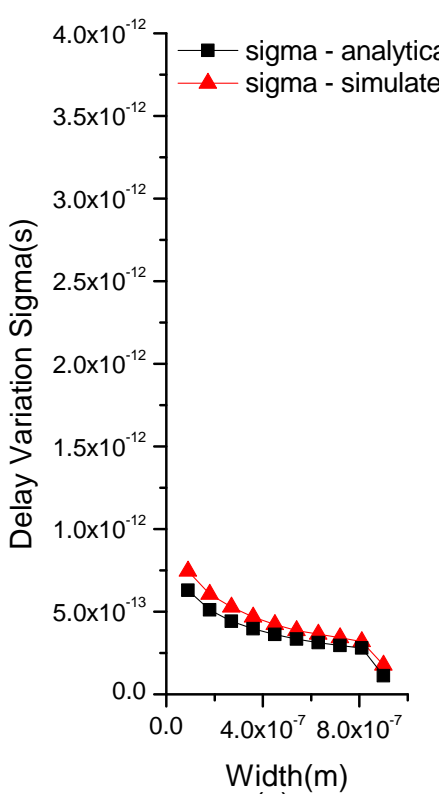

(a)

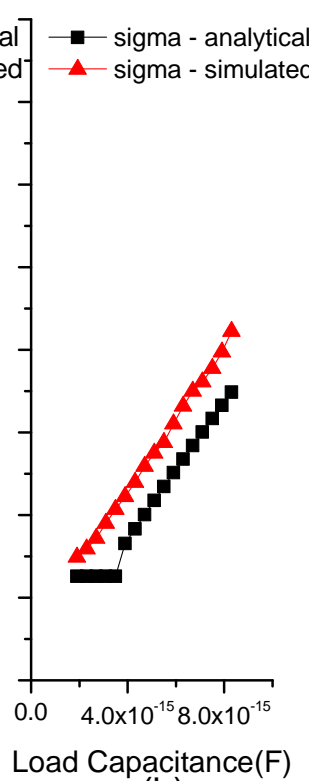

(b)

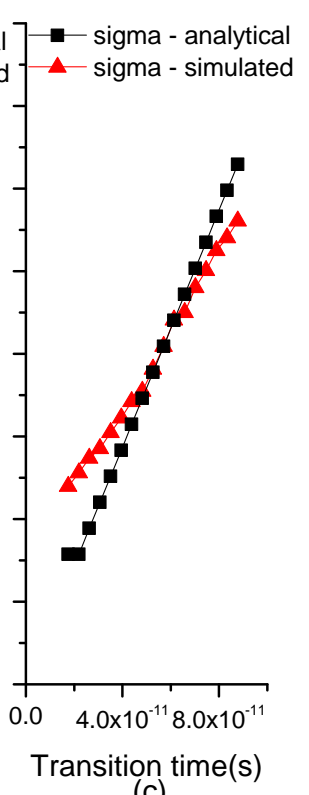

(c)

Figure 4.2: Inverter HL delay variation with varying (a)width, (b)capacitance, (c)transition time at $32 \mathrm{~nm}$ technology node.

The analysis for inverter low to high(LH) delay, $T_{p l h}$, is the same. Figures 4.3 and 4.4 show the variation in $T_{p l h}$ for varying widths, load capacitances and input transition times for $45 \mathrm{~nm}$ and $32 \mathrm{~nm}$ nodes respectively. As can be seen from Figures $4.1-4.4$, for the same amount of variation, delay variability in $32 \mathrm{~nm}$ is much higher than $45 \mathrm{~nm}$. According to ITRS [2], variation in $V_{t h}$ increases and nominal delay decreases with technology scaling. Thus $\sigma / \mu$ of delay only gets amplified.

The maximum difference in simulated and model estimated $\sigma / \mu$ is $0.68 \%$ while varying width, $0.47 \%$ while varying $C_{L}$ and $1.09 \%$ while varying $t_{r}$ for HL delays at $45 \mathrm{~nm}$ technology. The maximum difference in simulated and model estimated $\sigma / \mu$ is $1.7 \%$ while varying width, $2.34 \%$ while varying $C_{L}$ and $2.01 \%$ while varying $t_{r}$ for LH delays at $45 \mathrm{~nm}$ technology. The differences are higher in $32 \mathrm{~nm}$ technology. The maximum difference in simulated and model estimated $\sigma / \mu$ is $1.93 \%$ while varying width, $2.9 \%$ while varying $C_{L}$ and $2.8 \%$ while varying $t_{r}$ for HL delays at $32 \mathrm{~nm}$ technology. The maximum difference in simulated and model estimated $\sigma / \mu$ is $2.18 \%$ while varying width, $3.1 \%$ while varying $C_{L}$ and $2.8 \%$ while varying $t_{r}$ for LH delays at 32nm technology. 


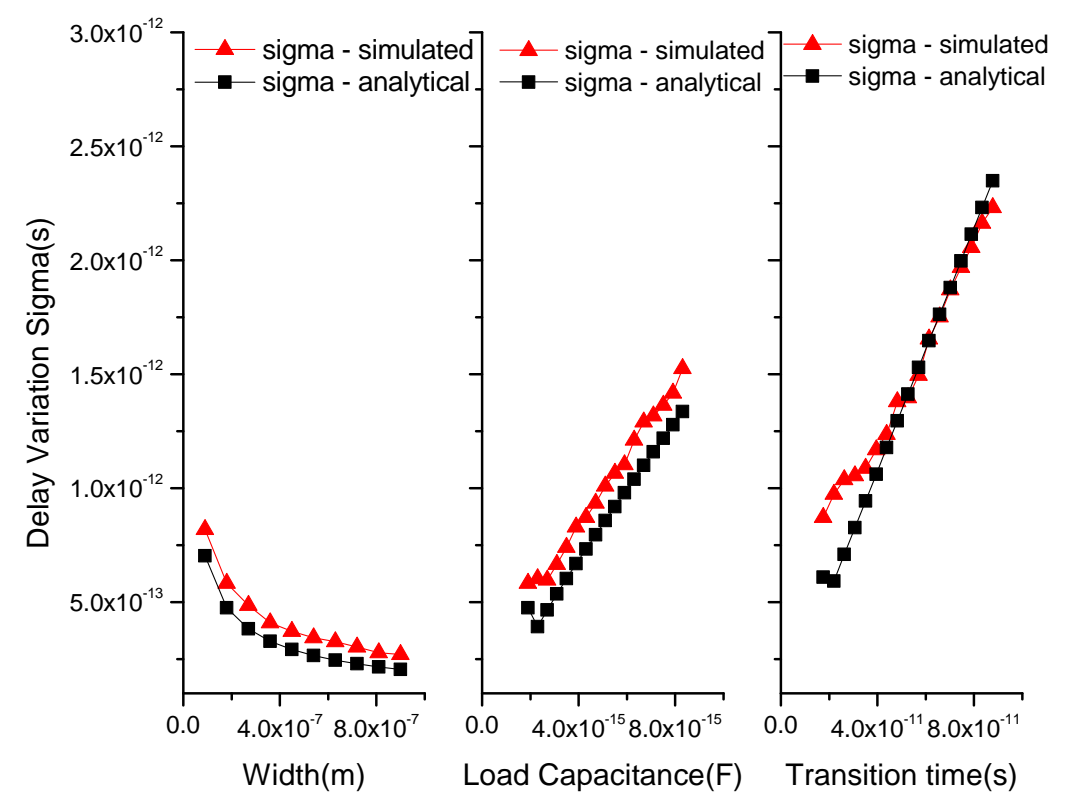

Figure 4.3: Inverter LH delay variation with varying width, capacitance, transition time at $45 \mathrm{~nm}$ technology node.

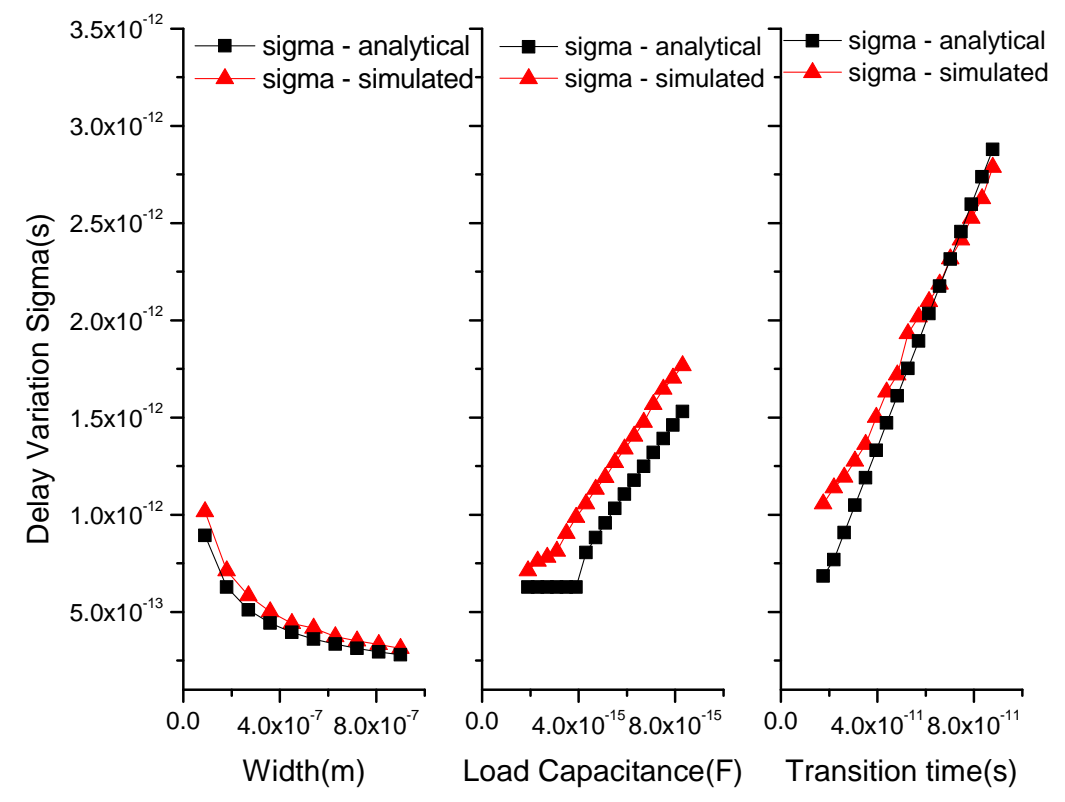

Figure 4.4: Inverter LH delay variation with varying width, capacitance, transition time at $32 \mathrm{~nm}$ technology node. 


\subsection{Delay Variability in NAND2 and NOR2 gates}

Delay variations in NAND and NOR gates can also be derived using equation (4.1). Delay variation for NAND2 high to low delay is given below. The variation depends on whether input is given to top transistor or bottom transistor of the stack.

Case 1. Input given to bottom transistor: When input is given to bottom transistor (M2 of Figure 3.8), $V_{t h}$ variation in any of the transistors in the stack affects delay variation.

1. Variation in $V_{t h}$ of bottom transistor: Partial derivative of NAND2 delay equation (3.10) with respect to $V_{t h}$ gives three solutions according to the input slew rate. $t_{\text {sat }}$ is time taken for the intermediate node voltage $V_{x}$ to discharge from initial value of $V_{D D}-V_{t h, M 1}$ to $V_{D S A T}$ and is given in equation (3.11). $t_{v x}$ is the time taken for $V_{x}$ to discharge from initial value of $V_{D D}-V_{t h, M 1}$ to $V_{x f}$, where $V_{x f}$ is the final voltage at node $X$ when $V_{o u t}$ is discharged to $V_{D D} / 2$. It is given in equations (3.13), (3.14) and (3.15).

$\mathbf{t}_{\mathbf{v x}}<\mathbf{t}_{\mathbf{r}}$ : For this case, input is very slow and $V_{x}$ reaches its final value before input reaches $V_{D D}$. The variation in delay is given as:

$$
\sigma_{T_{p}}=\left(\frac{t_{r}}{V_{D D}}+\frac{0.69 R_{1} C_{L}}{V_{D D}-V_{t h}}\right) \sigma_{V_{t h}}
$$

$\mathbf{t}_{\mathbf{s a t}}<\mathbf{t}_{\mathbf{r}}, \mathbf{t}_{\mathbf{v x}}>\mathbf{t}_{\mathbf{r}}$ : For this case, input is slow and $V_{x}$ reaches $V_{D S A T}$ before input reaches $V_{D D}$, but $V_{x}$ reaches its final value after input reaches $V_{D D}$. The variation in delay is given as:

$$
\sigma_{T_{p}}=\left(2 K_{x}\left(V_{D D}-V_{t h}\right) R_{1}\left(C_{L}+C_{x}\right)+\frac{\ln \left(V_{x, t r} / V_{x f}\right) R_{1}\left(C_{L}+C_{x}\right)}{\left(V_{D D}-V_{t h}\right)}\right) \sigma_{V_{t h}}
$$

where $K_{x}=\frac{\beta_{s} t_{r}\left[1+\lambda\left(V_{D S A T}-V_{D D}\right)\right]}{V_{D D} C_{L} V_{D S A T}(\alpha+1)}$.

$\mathbf{t}_{\mathbf{v x}}>\mathbf{t}_{\mathbf{r}}$ : For this case, input is fast and input reaches $V_{D D}$ before even $V_{x}$ reaches $V_{D S A T}$. The variation in delay is given as:

$$
\sigma_{T_{p}}=\left(\frac{t_{r}}{V_{D D}}+\frac{\ln \left(V_{D S A T} / V_{x f}\right) R_{1} C_{L}}{V_{D D}-V_{t h}}\right) \sigma_{V_{t h}}
$$

2. Variation in $V_{t h}$ of top transistor: The partial derivative of equation (3.10) with respect to $V_{t h, M 1}$ gives three possible solutions depending on the input transition time. 
$\mathbf{t}_{\mathbf{v x}}<\mathbf{t}_{\mathbf{r}}$ : For this case, input is very slow and $V_{x}$ reaches its final value before input reaches $V_{D D}$. The variation in delay is given as:

$$
\sigma_{T_{p}}=\left(\frac{t_{r}}{V_{D D} K_{y} \sqrt{\frac{\ln \left(V_{x f}\right)+C}{-K_{x}}}\left(V_{D D}-1 / \lambda+V_{t h, M 1}\right)}\right) \sigma_{V_{t h}} .
$$

where $K_{x}=\frac{\beta_{s} t_{r}\left[1+\lambda\left(V_{D S A T}-V_{D D}\right)\right]}{V_{D D} C_{L} V_{D S A T}(\alpha+1)}, K_{y}=\frac{\beta_{s} t_{r} \lambda}{C_{L}(\alpha+1) V_{D D}}, C=-K_{x}\left(\frac{V_{D D} t_{s a t}}{t_{r}}-V_{t h}\right)^{\alpha+1}-\ln \left(V_{D S A T}\right)$. $\mathbf{t}_{\text {sat }}<\mathbf{t}_{\mathbf{r}}, \mathbf{t}_{\mathbf{v x}}>\mathbf{t}_{\mathbf{r}}$ : For this case, input is slow and $V_{x}$ reaches $V_{D S A T}$ before input reaches $V_{D D}$, but reaches its final value after input reached $V_{D D}$. The variation in delay is given as:

$$
\sigma_{T_{p}}=\left(\frac{R_{1} C_{L} K_{x}}{K_{y} *\left(V_{D D}-1 / \lambda+V_{t h, M 1}\right)}\right) \sigma_{V_{t h}}
$$

$\mathbf{t}_{\mathbf{v x}}>\mathbf{t}_{\mathbf{r}}$ : For this case, input is fast and input reaches $V_{D D}$ before even $V_{x}$ reaches $V_{D S A T}$. The variation in delay is given as:

$$
\sigma_{T_{p}}=\left(\frac{t_{r}}{V_{D D} * \sqrt{K_{l o g}} K_{y}\left(V_{D D}-1 / \lambda+V_{t h, M 1}\right)}+\frac{0.69 R_{1} C_{L}}{V_{D D}-V_{t h, M 1}}\right) \sigma_{V_{t h}} .
$$

where where $K_{l o g}=\frac{1}{K_{y}} \ln \left[\frac{0.5 V_{D D}-\frac{1}{\lambda}}{V_{D D}-\frac{1}{\lambda}}\right]$.

Case 2. Input given to top transistor: When input is given to top transistor, delay depends only on $V_{t h}$ of top transistor. So variation in delay is given by inverter delay variation as in equations (4.2) or (4.3) depending on the region or operation when $V_{\text {out }}$ reaches $V_{D D} / 2$. So variation in bottom transistor should have almost no effect on the delay in this case.

Model Validation: Table 4.1 shows variation when input is given to top(M1) and bottom(M2) transistors. In each case $V_{t h}$ of only M1 or M2 is varied. NAND2 gate is loaded with FO10 and fanin is set at FO4. Width of gate is taken to be 4 times minimum size, that is width of PMOS transistors is 4 times minimum size and width of NMOS transistors is 8 times the minimum width. Amount of $V_{t h}$ variation added is calculated using the method in [32]. Simulated and model estimated results show that, when input is given to M1, delay variability depends only on $V_{t h}$ of M1 alone. But when input is given to M2, $V_{t h}$ of both M1 and M2 affect delay variability. Simulation results closely match with model estimated values. 


\begin{tabular}{|c|c|c|c|c|c|c|c|}
\hline Input transistor & Variation transistor & \multicolumn{3}{|c|}{ Simulated } & \multicolumn{3}{|c|}{ Analytical } \\
\hline & & $\mu(\mathrm{ps})$ & $\sigma(\mathrm{ps})$ & $\sigma / \mu \%$ & $\mu(\mathrm{ps})$ & $\sigma(\mathrm{ps})$ & $\sigma / \mu \%$ \\
\hline \multirow{3}{*}{ M1 } & M1 & 15.60 & 1.68 & 10.77 & 13.86 & 1.17 & 8.43 \\
& M2 & 15.50 & 0.34 & 2.19 & 13.86 & 0 & 0 \\
\hline \multirow{2}{*}{ M2 } & M1 & 16.50 & 0.91 & 5.52 & 16.14 & 0.97 & 6.01 \\
& M2 & 16.50 & 1.49 & 9.03 & 16.14 & 1.52 & 9.42 \\
\hline
\end{tabular}

Table 4.1: Variation numbers when input is given to top(M1) and bottom(M2) transistors of NAND2 gate, with $V_{t h}$ of one of them varying.

NAND2 HL delay variations when input is given to top and bottom transistors are plotted for 45nm technology in Figures 4.5 and 4.6. Figures show NAND2 delay variations with varying widths, load capacitances and transition times. The maximum difference in predicted and HSPICE simulated $\sigma / \mu$ is $1.7 \%$ when varying width, $1.4 \%$ when varying load capacitance and $1.7 \%$ when varying input transition time when input is given to top(M1) transistor. When input is given to bottom(M2) transistor, maximum difference between model estimated and HSPICE simulated $\sigma / \mu$ is $1.3 \%$ while varying width, $0.90 \%$ while varying load capacitance and $0.69 \%$ while varying input transition time. Thus in all cases the difference between estimated results and HSPICE simulation results is very small.

\subsection{Delay Variability in NAND3}

Similar analysis is used to derive equations for delay variability of NAND3. Delay variation depends on whether the input is given to the bottom, middle or top transistor.

Case 1. Input given to bottom transistor: When input is given to bottom transistor, $V_{t h}$ variation in top most transistor and bottom most transistor affects delay variability. Middle transistor variation by itself does not have significant effect on delay variability because its variation is compensated either by top or bottom transistors.

1. Variation in $V_{t h}$ of bottom transistor: Partial derivative of equation (3.20) with respect to $V_{t h}$ gives equations (4.6), (4.5) and (4.4) according to input transition time.

2. Variation in $V_{t h}$ of top transistor: Partial derivative of equation (3.20) with respect to 


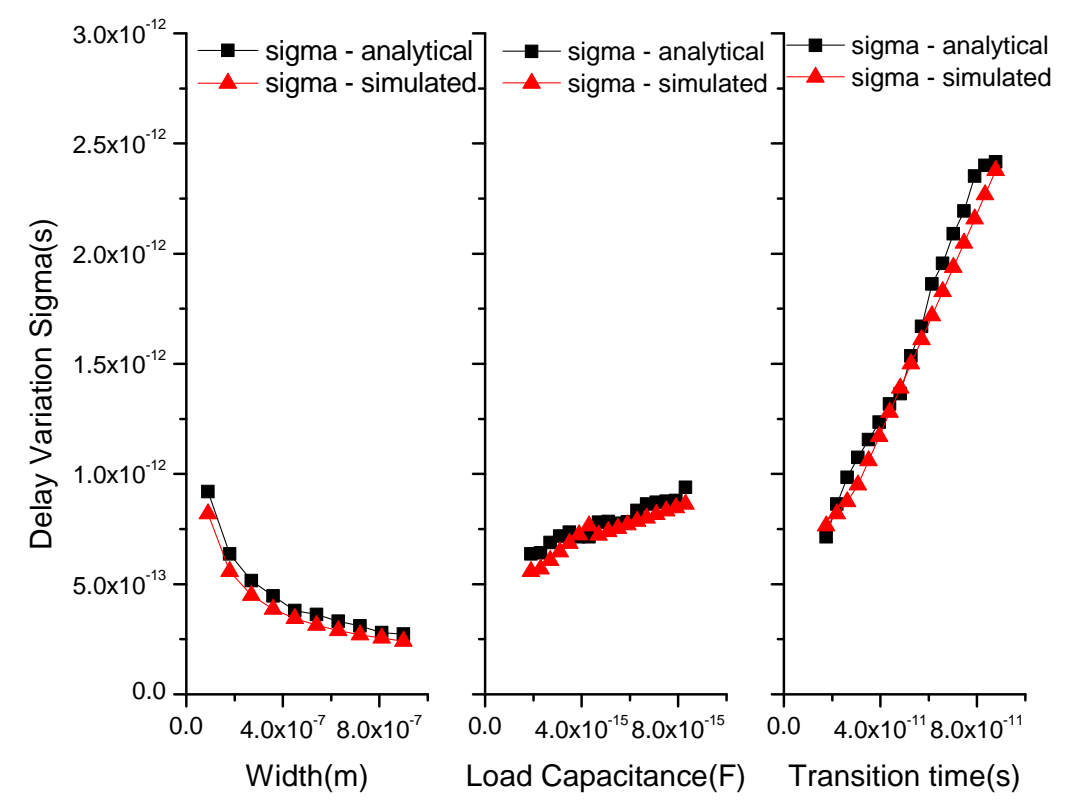

Figure 4.5: NAND2 gate HL delay variation with varying width, capacitance, transition time when input is given to $\mathrm{M} 2$ at $45 \mathrm{~nm}$ technology node.

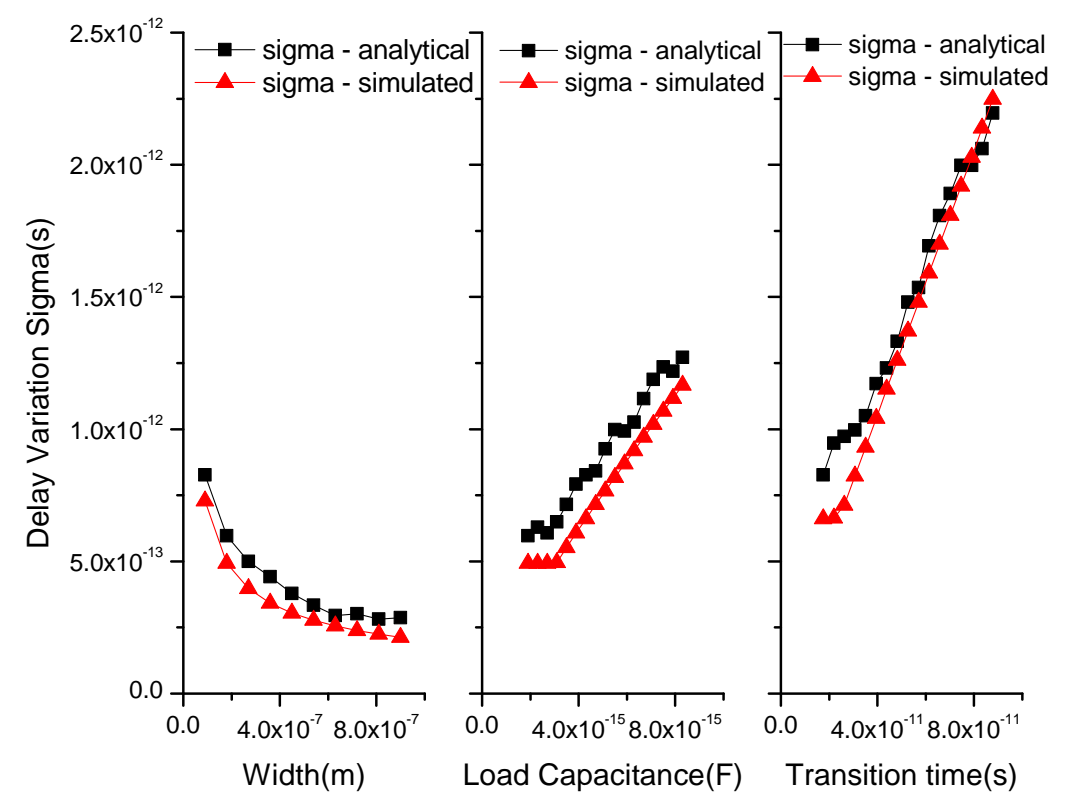

Figure 4.6: NAND2 gate HL delay variation with varying width, capacitance, transition time when input is given to M1 at $45 \mathrm{~nm}$ technology node. 
$V_{t h, M 1}$ for the case when $t_{s a t}>t_{r}$ gives

$$
\sigma_{T_{p}}=\left(\frac{t_{r}}{V_{D D} * \sqrt{K_{l o g}} K_{y}\left(V_{D D}-1 / \lambda+V_{t h, M 1}\right)}+\frac{0.69 R_{1}\left(C_{L}+C_{x}\right)}{V_{D D}-V_{t h, M 1}}+\frac{0.4 R_{2} C_{L}}{V_{D D}-V_{t h, M 1}}\right) \sigma_{V_{t h}} .
$$

For the other two cases when $t_{s a t}<t_{r}$ and $t_{v x}<t_{r}$, equations (4.5) and (4.4) hold true.

Case 2. Input given to middle transistor: When input is given to middle transistor, $V_{t h}$ variation in any of the top or middle transistors affects delay variation. Bottom most transistor does not affect output delay hence does not affect variability.

1. Variation in $V_{t h}$ of middle transistor: Partial derivative of equation (3.20) with respect to $V_{t h}$ gives equations similar to (4.4), (4.5) and (4.6) depending on input slew rate.

2. Variation in $V_{t h}$ of top transistor: Partial derivative of equation (3.20) with respect to $V_{t h, M 1}$ gives equations similar to (4.7), (4.8) and (4.9) according to input transition time.

Case 3. Input given to top transistor: When input is given to top transistor, delay depends only on $V_{t h}$ of top transistor. So variation in delay is given by inverter delay variation equation as in (4.2) or (4.3) depending on the region when $V_{\text {out }}$ reaches $V_{D D} / 2$. So variation in middle or bottom transistor should have almost no effect on the delay variation.

Model Validation: Table 4.2 shows variation when input is given to top(M1), middle(M2) and bottom(M3) transistors. In each case $V_{t h}$ of only M1, M2 or M3 transistor is varied. NAND3 gate considered is 4 times minimum length, that is PMOS transistor is 4 times minimum length and NMOS is 12 times minimum length. It is loaded with FO10 and fanin is FO4. Amount of $V_{t h}$ variation to be added is calculated using method from [32]. As expected, when input is given to M3 transistor, $V_{t h}$ variation in M1 and M3 results in considerable delay variability but variation in M2 transistor has almost no effect. When input is given to M2 transistor, it is similar to NAND2 gate. So variation in M1 and M2 transistors add to variability, but bottom transistor does not have any effect. When input is given to M1 transistor, variation in only M1 affects delay. Simulation results match very well with model estimated values. 


\begin{tabular}{|c|c|c|c|c|c|c|c|}
\hline Input transistor & Variation transistor & \multicolumn{3}{|c|}{ Simulated } & \multicolumn{3}{c|}{ Analytical } \\
\hline & & $\mu(\mathrm{ps})$ & $\sigma(\mathrm{ps})$ & $\sigma / \mu \%$ & $\mu(\mathrm{ps})$ & $\sigma(\mathrm{ps})$ & $\sigma / \mu \%$ \\
\hline \multirow{3}{*}{ M1 } & M1 & 15.29 & 0.63 & 4.14 & 15.29 & 0.51 & 3.34 \\
& M2 & 15.22 & 0.16 & 1.06 & 15.29 & $\sim$ & $\sim$ \\
& M3 & 15.19 & 0.14 & 0.94 & 15.29 & $\sim 0$ & $\sim$ \\
\hline \multirow{3}{*}{ M2 } & M1 & 17.66 & 0.32 & 1.83 & 16.57 & 0.64 & 3.86 \\
& M2 & 17.68 & 0.58 & 3.26 & 16.57 & 0.64 & 3.86 \\
& M3 & 17.62 & 0.11 & 0.62 & 16.57 & 0 & $\sim$ \\
\hline \multirow{3}{*}{ M3 } & M1 & 18.02 & 0.34 & 1.90 & 18.47 & 0.64 & 3.47 \\
& M2 & 18.00 & 0.21 & 1.16 & 18.47 & $\sim 0$ & $\sim$ \\
& M3 & 18.06 & 0.63 & 3.47 & 18.47 & 0.64 & 3.47 \\
\hline
\end{tabular}

Table 4.2: Variation numbers when input is given to top(M1), middle(M2) and bottom(M3) transistors of NAND3 gate, with $V_{t h}$ of one of them varying.

NAND3 delay variations at 45nm are plotted in Figures 4.7, 4.8 and 4.9 when input is given to M1, M2 and M3 transistors, respectively. Figures show delay variations for varying widths, load capacitances and input transition times. The maximum difference in predicted and HSPICE simulated $\sigma / \mu$ is $0.75 \%$ while varying width, $0.97 \%$ while varying load capacitance and $0.82 \%$ while varying input slew rate when input is given to M1. The maximum difference in predicted and HSPICE simulated $\sigma / \mu$ is $0.42 \%$ while varying width, $0.63 \%$ while varying load capacitance and $0.63 \%$ while varying input slew rate when input is given to M2. The maximum difference in predicted and HSPICE simulated $\sigma / \mu$ is $0.15 \%$ while varying width, $0.48 \%$ while varying load capacitance and $0.6 \%$ while varying input slew rate when input is given to M3.

Interestingly for large load capacitances, delay variability is high when input is given to M1 transistor than when given to M2 and M3 transistors for NAND3. Similar is the trend for NAND2. This is because, discharge rate of M1, M2 and M3 affect delay when input is given to M3 and discharge rate of M1 and M2 affect delay when input is given to M2. But when input is given to M1, output depends on discharge rate of only M1. When load is high, the current reaches maximum value in M1 increasing nominal delay to a large extent. As variability depends on nominal delay also, large loads result in high delay variability. 


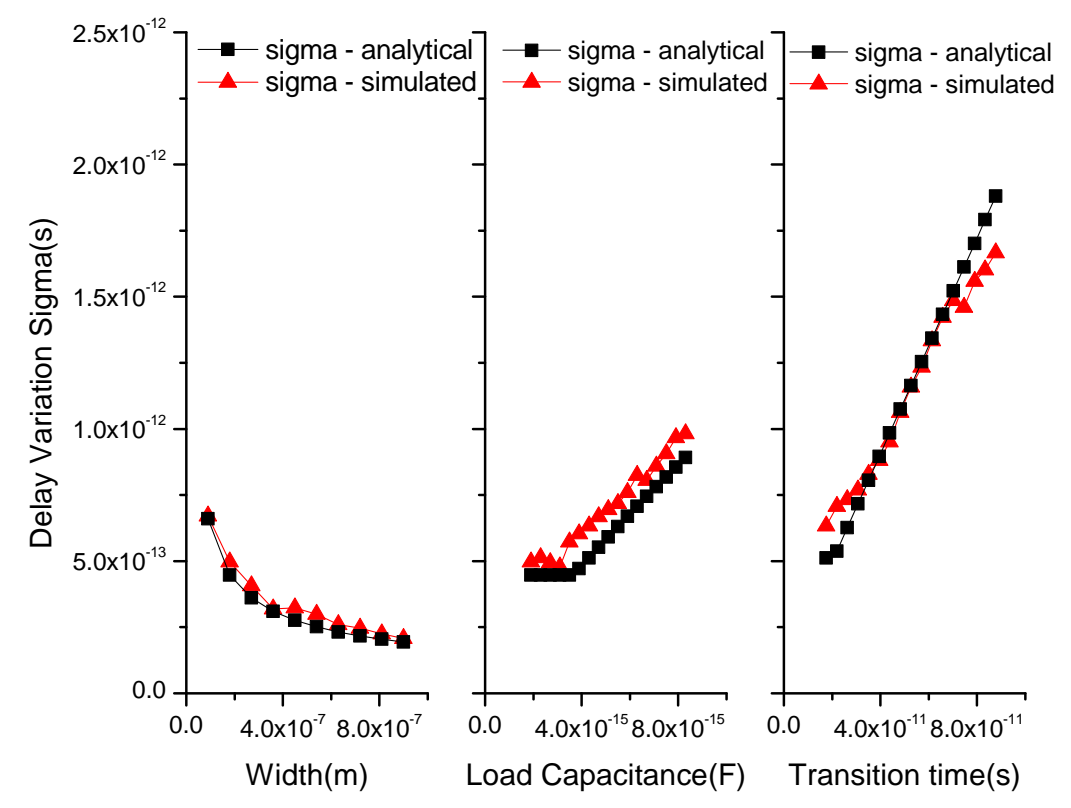

Figure 4.7: NAND3 gate HL delay variation with varying width, capacitance, transition time when input is given to M1 at $45 \mathrm{~nm}$ technology node.

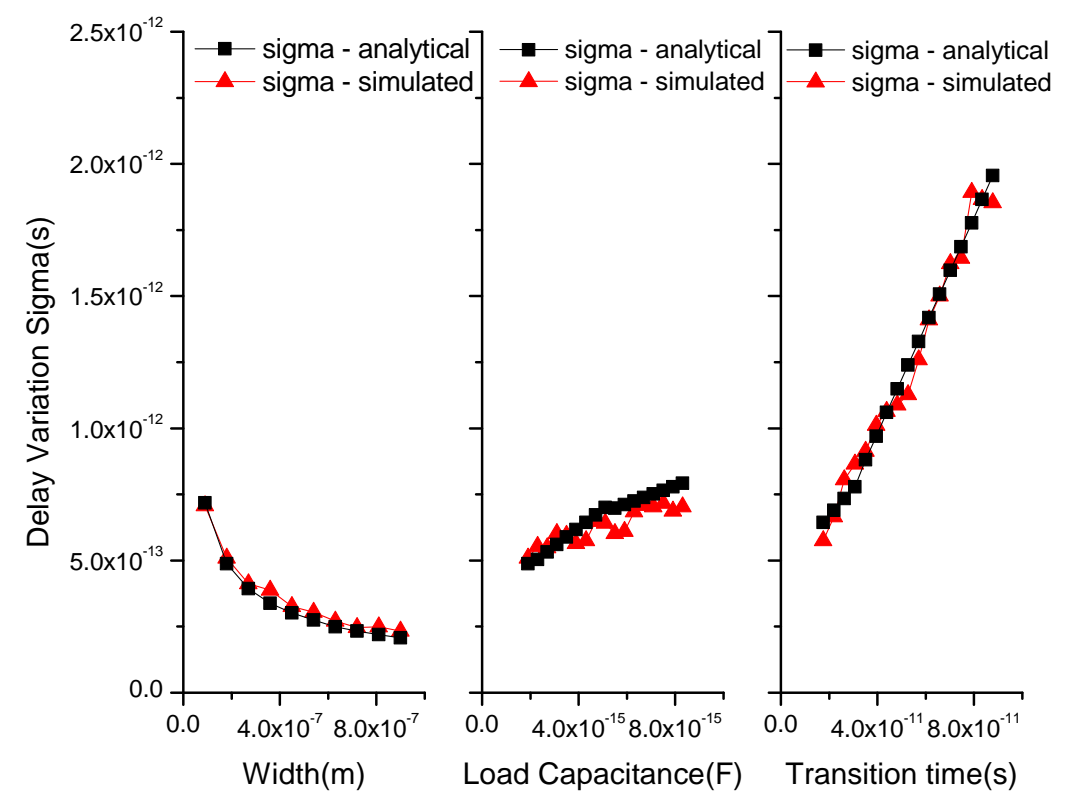

Figure 4.8: NAND3 gate HL delay variation with varying width, capacitance, transition time when input is given to M2 at $45 \mathrm{~nm}$ technology node. 


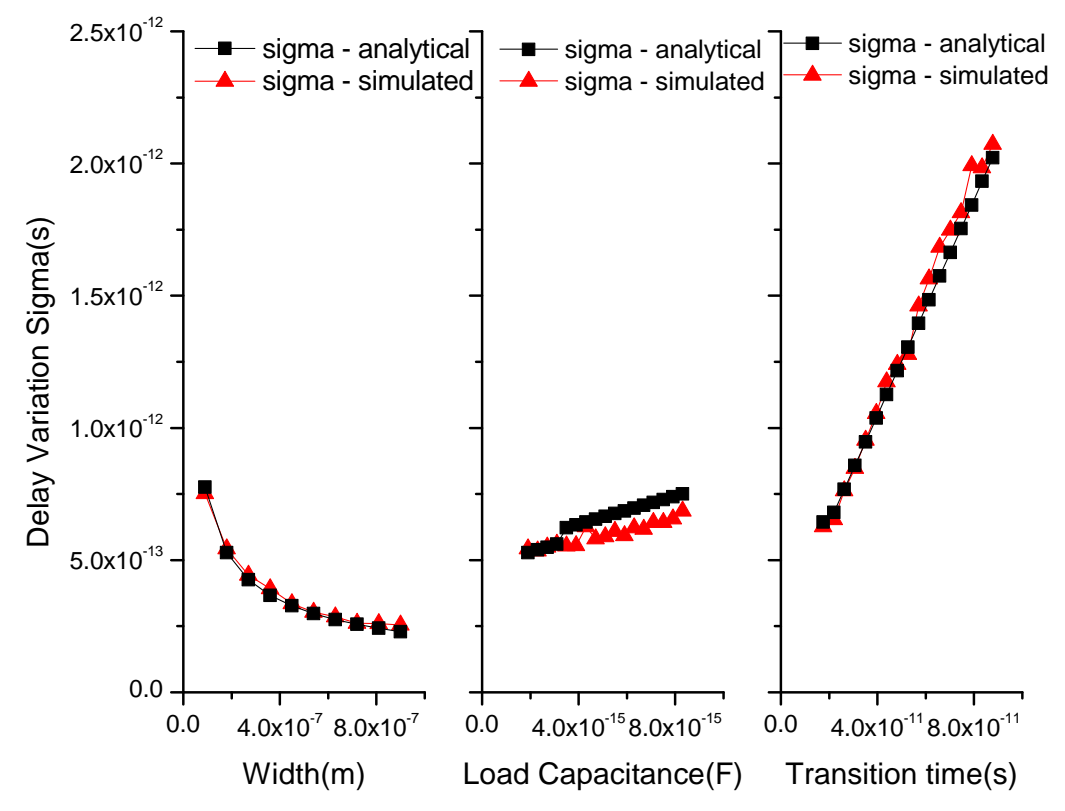

Figure 4.9: NAND3 gate HL delay variation with varying width, capacitance, transition time when input is given to M3 at $45 \mathrm{~nm}$ technology node. 


\section{Chapter 5}

\section{MODEL VALIDATION}

The proposed analytical model is applied to small circuits like XOR2 and Full Adder to estimate nominal delay and delay variability in Section 5.1. In Section 5.2, the model is tuned to match Nangate 45nm library [3] and applied to ISCAS' 85 benchmark circuits. The nominal delay estimated is in good agreement with simulated results; the maximum difference between analytically estimated delay and simulated delay for critical paths is less than $4 \%$. Also delay variability in critical paths is predicted for the ISCAS' 85 benchmark circuits.

\subsection{Small Circuits}

Delays are estimated using the proposed analytical model for XOR2 and Full Adder circuits and compared with HSPICE simulated values. The approach followed is to estimate the nominal delays of individual stages of these circuits and add them to get total circuit nominal delay. Variability is also found for each gate and since variations in each transistor is considered to be independent, equation (5.1) is used to estimate total circuit variability.

$$
\sigma_{\text {path }}=\sqrt{\sum \sigma_{\text {gate }}^{2}}
$$

Example 1. XOR2 gate: The circuit of an XOR2 gate is shown in Figure 5.1. The width of the NMOS device, $W_{n}$ is 4 times the minimum length. The width of PMOS device, $W_{p}$ is $W_{n}$ multiplied by the $p-n$ ratio given in Table 2.1. If NMOS or PMOS transistors are stacked, then their width is scaled according to size of the stack. This XOR2 gate is connected to inverters at both input and output as shown in Figure 5.2. The input patterns considered are, $A=0, B$ switching ; $B=0, A$ switching, since these patterns activate both the stages. The results are shown in Table 5.1 for $45 \mathrm{~nm}$ technology node.

\begin{tabular}{|c|c|c|c|c|c|c|}
\hline & \multicolumn{2}{|c|}{ Simulated Results } & \multicolumn{2}{|c|}{ Model Results } & & \\
\hline & $\mu(\mathrm{ps})$ & $\sigma(\mathrm{ps})$ & $\mu(\mathrm{ps})$ & $\sigma(\mathrm{ps})$ & $\sigma / \mu \%$ - simulated & $\sigma / \mu \%$ - model \\
\hline B-lh, O-lh & 19.86 & 1.48 & 19.74 & 1.28 & 7.45 & 6.48 \\
\hline B-hl, O-hl & 19.95 & 1.53 & 19.59 & 1.50 & 7.67 & 7.66 \\
\hline A-lh, O-lh & 17.25 & 1.44 & 17.45 & 1.28 & 8.35 & 7.34 \\
\hline A-hl, O-hl & 18.33 & 1.50 & 19.45 & 1.40 & 8.18 & 7.20 \\
\hline
\end{tabular}

Table 5.1: XOR2 gate nominal delay and delay variation values from HSPICE simulations and model estimates. 


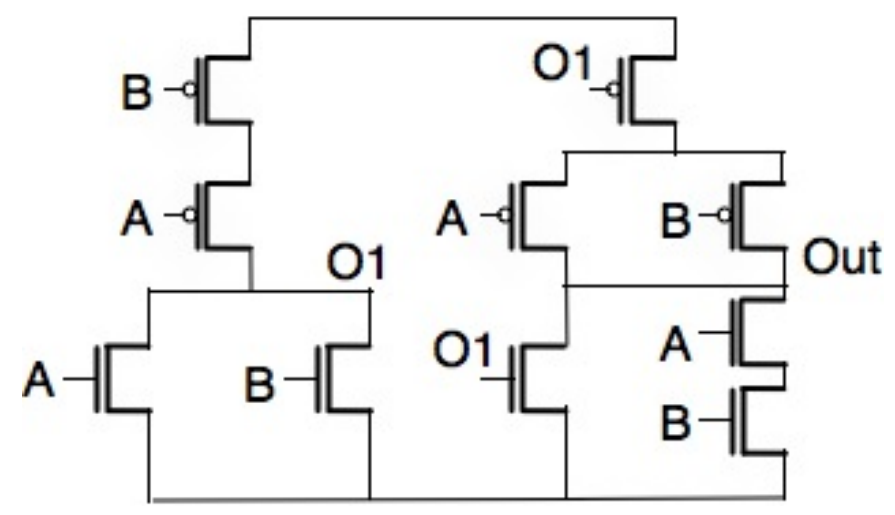

Figure 5.1: Schematic of XOR2 circuit

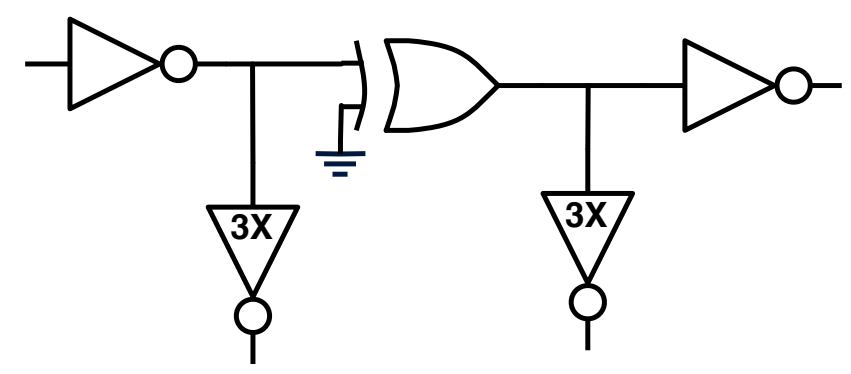

Figure 5.2: XOR2 gate with input and output loading with FO4.

Table 5.1 shows the that maximum error between simulated and estimated $\sigma / \mu$ is less than $1 \%$. Such an accurate prediction of nominal delay and delay variation has been possible because the model considers load capacitance, transition times and stacking effect.

Example 2. Full Adder: The full adder circuit is shown in Figure 5.3. The transistor widths considered are similar to XOR2 gate, namely, $W_{n}$ is 4 times minimum length and $W_{p}$ is $W_{n}$ multiplied by the $p-n$ ratio from Table 2.1. If NMOS or PMOS transistors are stacked, then their width is scaled according to number of transistors stacked. This full adder is connected to inverter at both input and output as shown in Figure 5.4.

The input patterns considered are, $A=1, C_{i}=0, B$ switching ; $B=0, C_{i}=1$, $A$ switching since these patterns activate both the stages. The results are shown in Table 5.2. The maximum difference between the $\sigma / \mu$ estimates and simulated value is $0.65 \%$. 


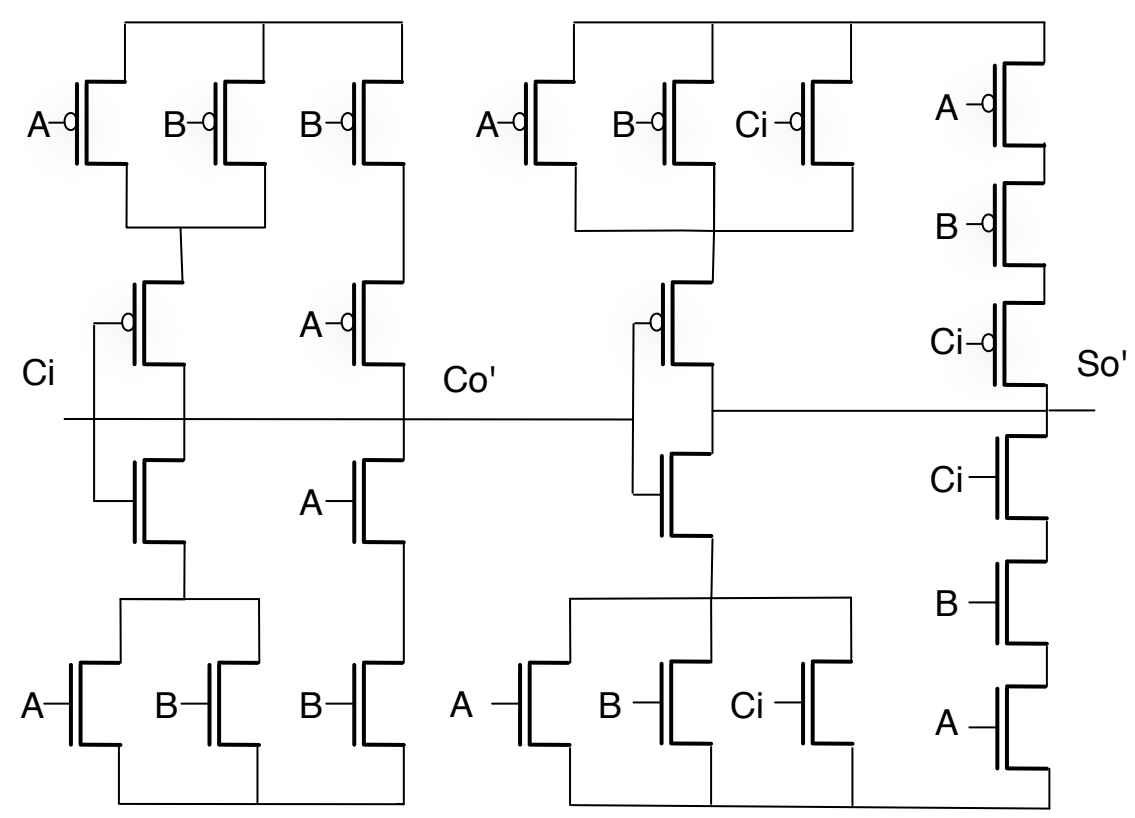

Figure 5.3: Mirror Adder structure of Full Adder

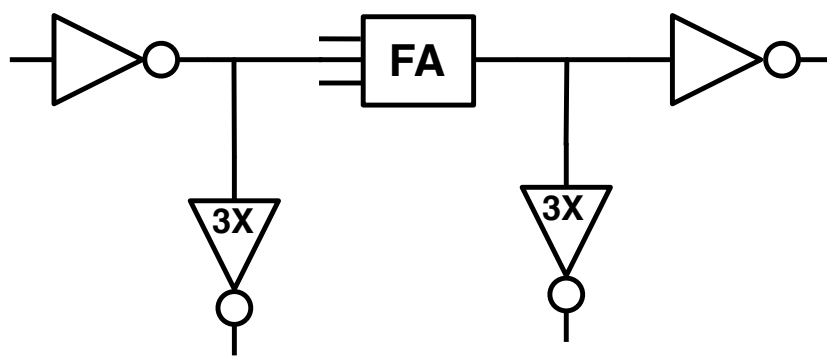

Figure 5.4: Full Adder with input and output loading with FO4.

\begin{tabular}{|c|c|c|c|c|c|c|}
\hline & \multicolumn{2}{|c|}{ Simulated Results } & \multicolumn{2}{|c|}{ Model Results } & & \\
\hline & $\mu(\mathrm{ps})$ & $\sigma(\mathrm{ps})$ & $\mu(\mathrm{ps})$ & $\sigma(\mathrm{ps})$ & $\sigma / \mu \%$ - simulated & $\sigma / \mu \%$ - model \\
\hline B-lh, O-lh & 24.73 & 1.726 & 22.63 & 1.611 & 6.98 & 7.12 \\
\hline B-hl, O-hl & 21.96 & 1.456 & 22.84 & 1.647 & 6.63 & 7.21 \\
\hline A-lh, O-lh & 24.96 & 1.868 & 23.65 & 1.681 & 7.48 & 7.11 \\
\hline A-hl, O-hl & 26.36 & 1.712 & 26.36 & 1.882 & 6.49 & 7.14 \\
\hline
\end{tabular}

Table 5.2: Full Adder nominal delay and delay variation values from simulated results and model estimated results.

\subsection{Application to ISCAS Benchmark Circuits}

Setup: The proposed model has been tuned to match the Nangate 45nm technology library [3].

The model is verified for gates with drive strength $\mathrm{X} 1$ in the library. Here input transition times range from $7.5 \mathrm{ps}$ to $600 \mathrm{ps}$ and load capacitance ranges from $0.4 \mathrm{fF}$ to $25.6 \mathrm{fF}$. 10 ISCAS bench- 
mark circuits with number of gates varying from 160 to 3512 were considered [1] To be able to simulate the circuits with [3] library, larger gates like NAND8, NAND9 are replaced with functionally equivalent smaller gates available in library. Synopsys primetime tool is used to extract critical paths form ISCAS circuits. The information of various paths is extracted from the output timing file of Synopsys primetime and then the proposed model estimated nominal delay and delay variability of each gate in the extracted paths. The nominal delay is summed up and variation is calculated according to equation (5.1).

\section{Comparison of nominal delay at $45 \mathrm{~nm}$ :}

The nominal delay values estimated using the model and the simulated results are shown in Table 5.3. Model predicted nominal delay for critical paths is in very good agreement with Synopsys primetime estimated results with maximum percentage error being 3.6\%. While the results here are for Nangate library [3], the model can easily be applied to other standard libraries.

\begin{tabular}{|c|c|c|c|c|c|}
\hline ISCAS circuit & Total Gates & $\begin{array}{c}\text { Gates in } \\
\text { critical path }\end{array}$ & $\begin{array}{r}\text { Simulated } \\
\text { nominal } \\
\text { delay (ns) }\end{array}$ & $\begin{array}{c}\text { Analytical } \\
\text { nominal } \\
\text { delay(ns) }\end{array}$ & Error \% \\
\hline C432 & 160 & 19 & 1.52 & 1.51 & $\mathbf{0 . 3 7}$ \\
\hline C499 & 202 & 12 & 1.25 & 1.27 & $\mathbf{0 . 9 3}$ \\
\hline C880 & 383 & 23 & 1.31 & 1.30 & $\mathbf{0 . 5 3}$ \\
\hline C1355 & 546 & 25 & 1.36 & 1.31 & $\mathbf{3 . 6 2}$ \\
\hline C1908 & 880 & 42 & 1.90 & 1.84 & $\mathbf{3 . 0 5}$ \\
\hline C2670 & 1113 & 31 & 2.08 & 2.13 & $\mathbf{2 . 3 6}$ \\
\hline C3540 & 1669 & 41 & 2.51 & 2.61 & $\mathbf{3 . 6 6}$ \\
\hline C5315 & 2307 & 48 & 2.25 & 2.28 & $\mathbf{0 . 9 9}$ \\
\hline C6288 & 2406 & 124 & 7.03 & 6.96 & $\mathbf{1 . 0 8}$ \\
\hline C7552 & 3512 & 42 & 1.94 & 1.88 & $\mathbf{3 . 2 4}$ \\
\hline
\end{tabular}

Table 5.3: Comparison of nominal delay estimation for all the ISCAS' 85 benchmark circuits.

\section{Variation prediction with the model:}


The delay variability of critical paths of the ISCAS benchmark circuits is estimated. Amount of $V_{t h}$ variation added is $50 \mathrm{mV}$ for a transistor of twice the minimum length and $\sigma_{V_{t h}} \propto \frac{1}{\sqrt{W}}$, where $W$ is the width of transistor. Table 5.4 shows delay variation for all the ISCAS benchmark circuits. The average variation is $1.7 \%$. The time taken to estimate variability with the model is a very small fraction of the time taken to run SPICE simulations.

\begin{tabular}{|c|c|c|c|}
\hline ISCAS circuit & Nominal Delay(ps) & Variation (ps) & $\sigma / \mu \%$ \\
\hline C432 & 1512.70 & 37.56 & 2.48 \\
\hline C499 & 1244.10 & 33.88 & 2.68 \\
\hline C880 & 1293.00 & 26.16 & 2.01 \\
\hline C1355 & 1321.70 & 27.11 & 2.07 \\
\hline C1908 & 1840.10 & 32.87 & 1.78 \\
\hline C2670 & 2141.90 & 27.13 & 1.27 \\
\hline C3540 & 2600.80 & 37.97 & 1.46 \\
\hline C5315 & 2261.50 & 27.33 & 1.20 \\
\hline C6288 & 6776.90 & 48.59 & 0.70 \\
\hline C7552 & 1875.10 & 24.18 & 1.34 \\
\hline
\end{tabular}

Table 5.4: Variation prediction for critical paths in ISCAS' 85 benchmark circuits

The variation is small in these circuits because of averaging out of random variations in these long paths.

\subsubsection{Effects of Variability}

The proposed analytical model is used to identify possible timing violations early in the design flow.

Setup time violations: Setup time violations are caused by variations in critical paths. Variability is low in these paths because of averaging effect. However, paths with slightly smaller delay can have larger variability and can become critical. Figure 5.5 shows the delay distribution graph for $\mathrm{C} 880$ benchmark circuit at nominal conditions and with $V_{t h}$ variations. As it can be seen from the graph the distribution widens because of variations and the number of critical paths increase. Some of the non-critical paths at nominal conditions have now become critical. 
The number of shortest paths also increase. The minimum delay decreased and this can cause a hold violation.

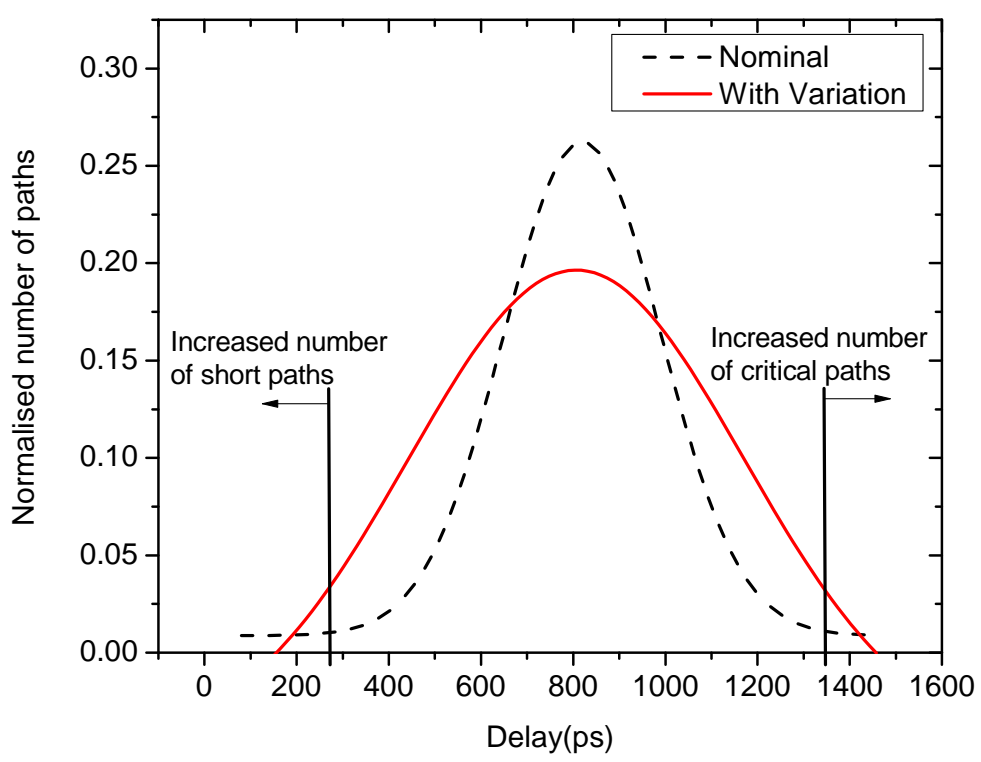

Figure 5.5: Delay distribution curve for $\mathrm{C} 880$ benchmark circuit at nominal and with variations.

Similar trend is seen in another circuit ISCAS C7552. Here the critical path has a nominal delay of $1885.4 \mathrm{ps}$ and paths with $5 \%$ less than critical path delay are also considered as critical paths. Consider Path-2 with a delay of $1787.3 \mathrm{ps}$, that is $5.2 \%$ smaller than the critical path and so not considered to be critical. Now with a slight $V_{t h}$ variation of $\sigma_{V_{t h}}=10 \mathrm{mV}$ (for a transistor with width twice the minimum length and $\sigma_{V_{t h}}$ varying with the relation $\sigma_{V_{t h}} \propto \frac{1}{\sqrt{W_{n}}}$ ), the worst case delay $(\mu+3 \sigma)$ of the critical path is $1900.5 \mathrm{ps}$ and of Path- 2 is $1806.8 \mathrm{ps}$, which is within $5 \%$ of the critical path delay. Hence Path-2 now is also critical. Similarly, Path-3 with nominal delay of $1777.7 \mathrm{ps}$ becomes critical for $\sigma_{V_{t h}}=30 \mathrm{mV}$. Figure 5.6 plots the worst case delays of these paths as a function of $\sigma_{V_{t h}}$. The plot also shows that as $\sigma_{V_{t h}}$ increases, the number of critical paths increases. The proposed model can thus help easily identify paths that may become critical due to $V_{t h}$ variations, early in the design phase and without time consuming Monte Carlo simulations. 

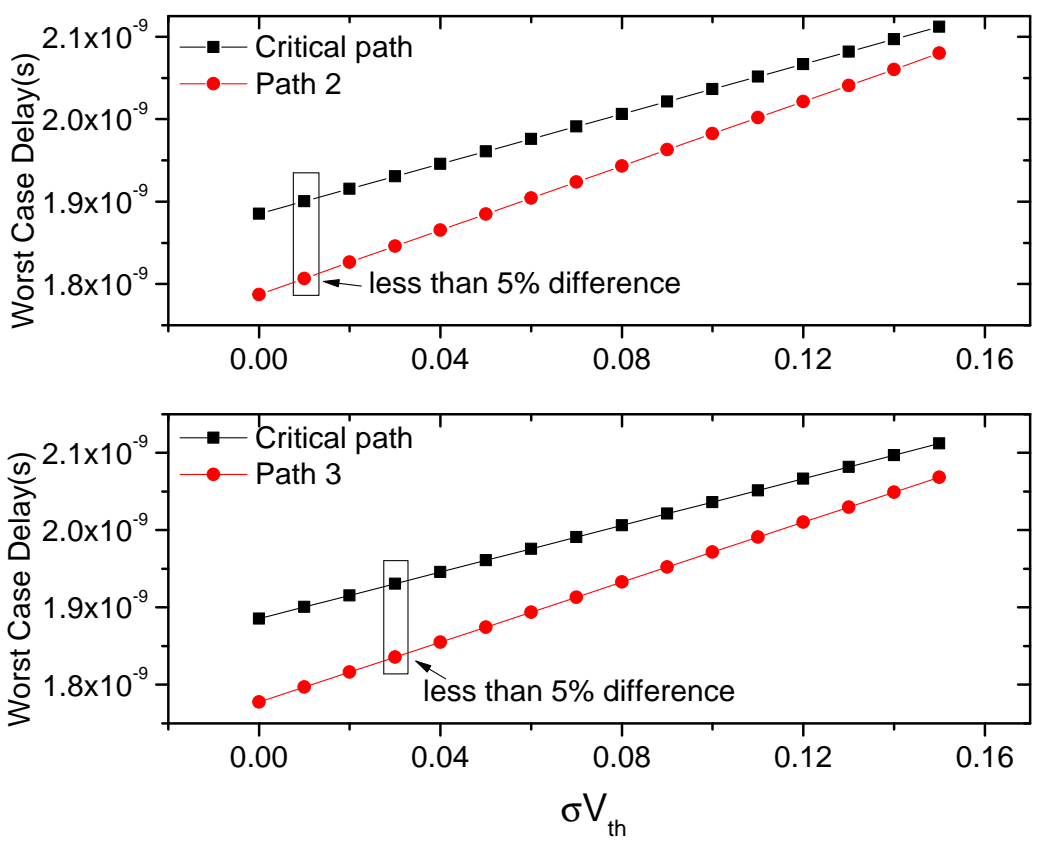

Figure 5.6: Non-critical path becoming critical in light of $V_{t h}$ variation.

Hold time violations: The delay variability is small in long paths because of averaging effect. But in smaller paths of the circuit, variation is considerable and the contaminated delays $(\mu-$ $3 \sigma$ ) can cause hold time violations.

The proposed model is applied to two ISCAS benchmark circuits, C5315 and C2670, to estimate the nominal delay and delay variability of the shortest paths. Hold time is assumed to be $15 \mathrm{ps}$ for D-Flip Flop from [3] for reasonable data and clock transition times. There are no hold violations under nominal conditions. But as variation in $V_{t h}$ increases, contaminated delays of many paths fall below hold time. Figure 5.7 shows the number of possible hold violations with varying $\sigma_{V_{t h}}$. For instance, when $\sigma_{V_{t h}}=50 \mathrm{mV}$, there are 12 hold violations in C5315 and 8 hold violations in C2670.

To avoid the hold time violations, design can be quickly modified by adding buffers to the identified shortest paths and the new design is verified for timing violations by the proposed model. For one of the failing paths in the C5315 ISCAS benchmark circuit, the nominal delay is $19.2 \mathrm{ps}$ and path length is 1 gate. With $\sigma_{V_{t h}}$ of $50 \mathrm{mV}$, delay variation is $1.58 \mathrm{ps}$, causing a hold violation. But with the addition of a buffer(two inverters), nominal delay is now 63.1ps and 
variation is now 3.06ps. Similarly for all the failing paths buffers are added and it is observed that all the possible hold violations are eliminated. Thus in this way the proposed model can be integrated into the design flow to account for variability during early stages of the design.

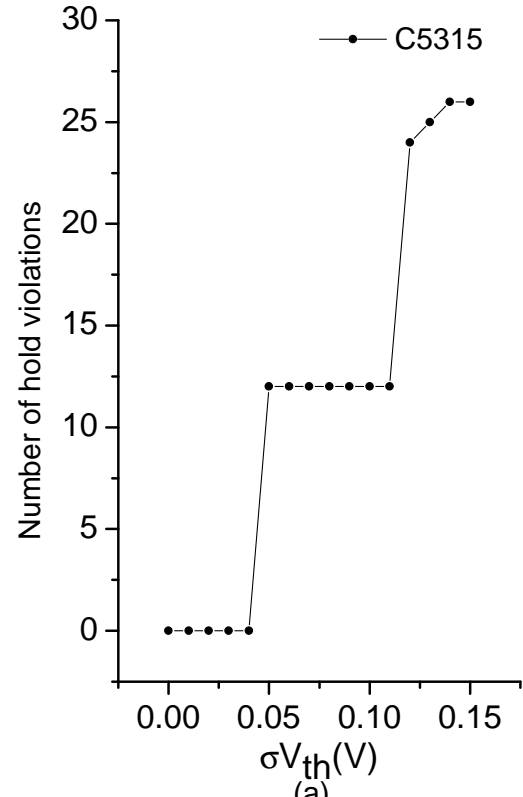

(a)

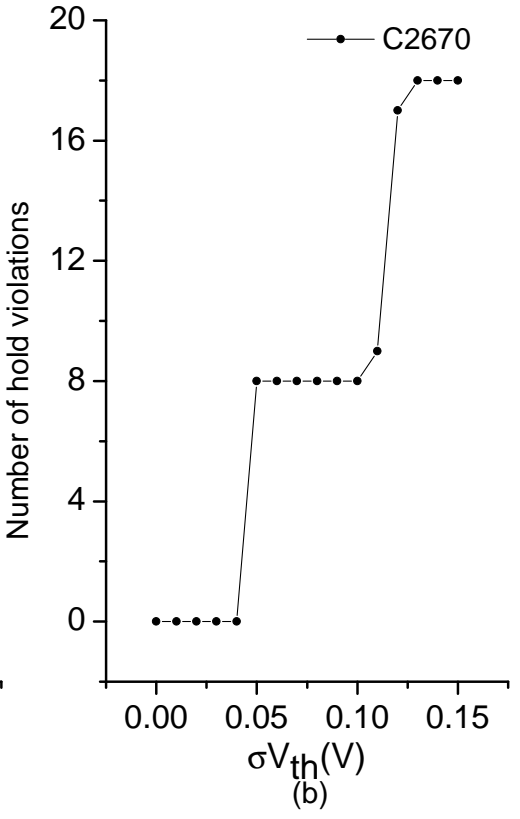

(b)

Figure 5.7: Number of paths that can cause hold time violations because of $V_{t h}$ variations in (a) C5315, (b) C2670, ISCAS benchmark circuit at 45nm technology node. 


\section{Chapter 6}

\section{CONCLUSIONS}

\subsection{Summary}

In this thesis, an accurate analytical model to predict nominal delay and delay variability of CMOS circuits is proposed. The model is comprehensive and enables a quick estimate of variability for large CMOS circuits. It can be integrated with an existing design flow to facilitate design of robust circuits in scaled technologies.

First a nominal delay model is developed for an inverter that considers factors such as gate width, load capacitance and input transition time. The average error compared to HSPICE simulated results is $-1.17 \%$ for varying width, load capacitance and input transition time at $45 \mathrm{~nm}$ technology node. The model is then extended to gates with stacked transistors like NAND and NOR. In such gates, the delay is also a function of the position of the transistor with switching input. The average error is $0.31 \%$ for varying width, load capacitance and input transition time at $45 \mathrm{~nm}$ technology for NAND2 gate.

Next, the model for delay variability because of varying $V_{t h}$ is derived for an inverter. The variability of a gate is directly proportional to $t_{r}$ except when loaded with large load capacitance. The maximum difference between estimated and simulated $\sigma / \mu$ is $1.09 \%$ for varying width, load capacitance and input transition time at $45 \mathrm{~nm}$ technology. The model is extended to handle stacked transistors. The maximum difference between estimated and simulated $\sigma / \mu$ is $1.7 \%$ for varying width, load capacitance and input transition time at $45 \mathrm{~nm}$ technology for NAND2 gate.

The proposed model is applied to complex gates like XOR and Full adder. Model estimated $\sigma / \mu$ matches HSPICE simulated results within $0.65 \%$ error. The model is then applied to complex ISCAS' 85 benchmark circuits. The nominal delay estimates are within $4 \%$ difference when compared to Synopsys primetime estimated results. The variability estimates for ISCAS' 85 benchmark circuits are done with in fraction of time compared to HSPICE simulations. It is also observed that, variability in non-critical paths can be more than that of critical path and the worst case delay $(\mu+3 \sigma)$ can be larger for non-critical paths. Also the rate of possible hold violations increases rapidly with increasing $V_{t h}$ variations. These kind of analysis 
were quickly done using the proposed model. Using HSPICE would have been impractical.

Thus the proposed model reduces time and effort to analyze variability of complex circuits without compromising on the accuracy.

\subsection{Future Work}

The model has been developed for all gates at $45 \mathrm{~nm}$ technology and for inverter at $32 \mathrm{~nm}$ technology. It can be further extended to $22 \mathrm{~nm}, 16 \mathrm{~nm}$ and $12 \mathrm{~nm}$ technology nodes. Variability is high at these technology nodes and $V_{t h}$ variation is not the only cause. Along with $V_{t h}$ variation, other parameter like width of transistors has to be taken into account. Variations in length and width become prominent at these technology nodes because of small feature size. The model can be extended to account for these variations also. 


\section{REFERENCES}

[1] ISCAS'85. http://dropzone.tamu.edu/ xiang/iscas.html.

[2] ITRS. http://public.itrs.net/.

[3] Nangate. http://www.nangate.net/.

[4] PTM. http://ptm.asu.edu/.

[5] M. Alam, K. Kang, B.C. Paul, and K. Roy. Reliability- and Process-Variation Aware Design of VLSI Circuits. 14th International Symposium on the Physical and Failure Analysis of Integrated Circuits, 2007. IPFA 2007, pages 17 -25, July 2007.

[6] M. Alioto, G. Palumbo, and M. Pennisi. Understanding the effect of process variations on the delay of static and domino logic. IEEE Transactions on Very Large Scale Integration (VLSI) Systems, 18:697-710, May 2010.

[7] A. Asenov, A.R. Brown, J.H. Davies, S. Kaya, and G. Slavcheva. Simulation of intrinsic parameter fluctuations in decananometer and nanometer-scale MOSFETs. IEEE Transactions on Electron Devices, 50(9):1837 - 1852, Sept. 2003.

[8] A. Asenov, S. Kaya, and J.H. Davies. Intrinsic threshold voltage fluctuations in decanano MOSFETs due to local oxide thickness variations. IEEE Transactions on Electron Devices, 49(1):112 -119, Jan 2002.

[9] S. Borkar. Circuit Techniques for Subthreshold Leakage Avoidance, Control, and Tolerance. Proc. Intl Electron Devices Meeting (IEDM 2004), IEEE Press, pages 421-424.

[10] S. Borkar. Designing reliable systems from unreliable components: the challenges of transistor variability and degradation. IEEE Micro, 25(6):10 - 16, Nov.-Dec. 2005.

[11] E. Cassan, P. Dollfus, S. Galdin, and P. Hesto. Calculation of direct tunnelling gate current through ultrathin oxide and oxide/nitride stacks in MOSFETs and H-MOSFETs. Microelectron Reliability, 40:585 - 588, 2000.

[12] M. Chandhok, K. Frasure, E.S. Putna, T. Younkin, W. Rachmady, U. Shah, and W. Yueh. Improvement in linewidth roughness by postprocessing. Journal of Vacuum Science Technology B: Microelectronics and Nanometer Structures, 26(6):2265 -2270, Nov. 2008.

[13] B. H. Calhoun et al. Digital circuit design challenges and opportunities in the era of nanoscale CMOS. Proceedings of the IEEE, 96(2):343-365, Feb 2008.

[14] D. L. Goldfarb et al. Effect of thin-film imaging on line edge roughness transfer to underlayers during etch processes. Journal of Vacuum Science Technology B: Microelectronics and Nanometer Structures, 22(2):647 -653, Mar 2004. 
[15] J. Tschanz et al. Effectiveness of Adaptive Supply Voltage and Body Bias for Reducing Impact of Parameter Variations in Low Power and High Performance Microprocessors. IEEE J. Solid-State Circuits, 38(5):826-829, May 2003.

[16] M. Niwa et al. Atomic order planarization ultrathin $\mathrm{SiO} 2 / \mathrm{Si}(001)$ interface. Applied Physics Letters, 63(5):675 -677, Aug 1993.

[17] S. Borkar et al. Parameter Variations and Impact on Circuits and Microarchitecture. Proc. 40th Design Automation Conf. (DAC 03), pages 338-342, 2003.

[18] S. Ghosh, S. Bhunia, and K. Roy. CRISTA: A New Paradigm for Low-Power, VariationTolerant, and Adaptive Circuit Synthesis Using Critical Path Isolation. IEEE Transactions Computer-Aided Design of Integrated Circuits and Systems, 26:1947-1956, Nov 2007.

[19] R.W. Keyes. Physical limits in digital electronics. Proceedings of the IEEE, 63(5):740 767, May 1975.

[20] P. Liu and Y.B. Kim. An accurate timing model for nano CMOS circuit considering statistical process variation. IEEE International SoC Design Conference(ISOCC), pages 269-272, 2007.

[21] P. Liu, Y.B. Kim, and Y.L. Lee. An Accurate Analytical Propagation Delay Model of Nano CMOS Circuits. IEEE International SoC Design Conference(ISOCC), pages 200203, 2007.

[22] T. Mizuno, J. Okumtura, and A. Toriumi. Experimental study of threshold voltage fluctuation due to statistical variation of channel dopant number in MOSFET's. IEEE Transactions on Electron Devices, 41(11):2216 -2221, Nov 1994.

[23] S. Nassif, K. Bernstein, D.J. Frank, A. Gattiker, W. Haensch, B.L. Ji, E. Nowak, D. Pearson, and N.J. Rohrer. High Performance CMOS Variability in the $65 \mathrm{~nm}$ Regime and Beyond. Electron Devices Meeting, 2007. IEDM 2007. IEEE International, pages 569 -571 , Dec. 2007.

[24] A. T. Putra, A. Nishida, S. Kamohara, and T. Hiramoto. Random Vth variation indueced by gate edge fluctuations in nanoscale MOSFETs. Silicon Nanoelectronics Workshop, pages 73-74, 2007.

[25] J.M. Rabaey. Digital Integrated Circuits: A Design Perspective. Englewood Cliffs, NJ: Prentice-Hall, 1996.

[26] T. Sakurai and A.R.Newton. Delay analysis of series-connected MOSFET circuits. IEEE J.Solid-State Circuits, 26:122-131, 1991. 
[27] T. Sakurai and A.R. Newton. Alpha-Power law MOSFET model and its application to CMOS inverter delay and other formulas. IEEE J. Solid-State Circuits, 25:584-594, 1990.

[28] S. R. Sarangi, B. Greskamp, R. Teodorescu, J. Nakano, A. Tiwari, and J. Torrellas. VARIUS: A model of process variation and resulting timing errors for microarchitects. IEEE Transactions on Semiconductor Manufacturing, 21:3-13, 2008.

[29] K. Yamaguchi T. Hagivaga and S. Asai. Threshold voltage variation in very small MOS transistors due to local dopant fluctuations. Proc. Symp. VLSI Technol., Dig. Tech. Papers, pages 46-47, 1982.

[30] D. Z.-Y. Ting, E. S. Daniel, and T. C. McGill. Interface roughness effects in ultrathin gate oxides. VLSI System Design, 8:47 - 51, 1998.

[31] Y. Wang and M. Zwolinski. Analytical transient response and propagation delay model for nanoscale CMOS inverter. IEEE International Symposium on Circuits and Systems, pages 2998 - 3001, May 2009.

[32] Y. Ye, S. Gummalla, C. Wang, C. Chakrabarti, and Y. Cao. Random Variability Modeling and its Impact on Scaled CMOS Circuits. J. Computational Electronics, pages 108-113, 2010.

[33] Y. Ye, F. Liu, M. Chen, S. Nassif, and Y. Cao. Statistical Modeling and Simulation of Threshold Variation Under Random Dopant Fluctuations and Line-Edge Roughness. IEEE Transactions on Very Large Scale Integration (VLSI) Systems, (99):1 -10, 2010. 